Technical Report

$$
\begin{aligned}
& \text { LEWIS GRANT } \\
& \text { AS-3y-CR } \\
& 3 / 4 / 2 \\
& 158
\end{aligned}
$$

\title{
Elliptic Jets. Part 2. Dynamics of Coherent Structures: Pairing
}

Sponsored by:

NASA Lewis Research Center

Grant Number: NAG 3-639

Principal Investigator:

Fazle Hussain

Cullen Distinguished Professor

Mechanical Engineering Department

University of Houston

Houston, Texas 77204-4792

February 24, 1992

(NASA-CR-189902) ELLIPTIC JETS, PART 2. DYNAMICS OF COAERENT STRUCTURES: PAIRING (Houston Univ.) $58 \mathrm{P}$

CSCL 200

N92-20372 


\title{
Elliptic Jets. Part 2. Dynamics of Coherent Structures: Pairing
}

\author{
By Hyder S. Husain and Fazle Hussain \\ Department of Mechanical Engineering, University of Houston, Texas 77204-4792
}

\begin{abstract}
The dynamics of coherent structure interactions, in particular the jet column mode of vortex pairing, in the near field of an elliptic jet have been investigated using hot-wire measurements and flow visualization. A 2:1 aspect-ratio jet with an initially laminar boundary layer and a constant momentum thickness all around the nozzle exit perimeter is used for this study. While detailed hotwire measurements were made in air at a Reynolds number $\operatorname{Re}_{\mathrm{De}}\left(\equiv \mathrm{U}_{\mathrm{e}} \mathrm{D}_{\mathrm{c}} / v\right)=3.2 \times 10^{4}$, flow visualization was performed in water at a lower $\operatorname{Re}_{\mathrm{De}}=1.7 \times 10^{4}$; here $U_{c}$ is the exit speed and $D_{c}$ is the equivalent diameter of the nozzle exit cross-section. Excitation at the stable pairing mode induced successive pairings to occur periodically at the same location, allowing phase-locked measurements using a local trigger sensor. Coherent structures were educed at different phases of pairing in the planes of both the major and minor axes. These are compared with corresponding data in a circular jet, educed similarly.

Pairing interactions are found to be quite different from those in a circular jet. Due to nomplanar and nonuniform self-induction of elliptical vortical structures and the consequent effect on mutual induction, pairing of elliptic vortices in the jet column does not occur uniformly around the entire perimeter, unlike in a circular jet. Merger occurs only in the initial major-axis plane through an entanglement process, while in the initial minor-axis plane, the trailing vortex rushes through the leading vortex without pairing and then breaks down violently. These motions produce considerably greater entrainment and mixing than in circular or plane jets. From distributions of dynamical properties over the extent of cohercnt structures, the production mechanism is explained in tcrms of the longitudinal vortices connecting the elliptic structures. Time-average measures and their modification by controlled excitation are also discussed in terms of coherent structure dynamics. A significant space in this paper is devoted to documenting phase-dependent and time-average flow measures; these new results should serve as target data for numerical simulations. Further details are given by Husain (1984).
\end{abstract}




\section{Introduction}

Pairing is a dynamically significant interaction of large-scale vortical structures in shear layers, jets and other turbulent shear flows. Large-scale engulfment, and enlanced mixing and transport of momentum (both co- and counter-gradient) can occur during pairing (Winant \& Browand 1974; Hussain 1981; Laufer 1983; Ho \& Huerre 1984). In many flow situations, such as impinging jets, turbulent boundary layers and wall jets, large-scale organized structures can induce flow separation. Pairing of the structures produces higher circulation structures, which in turn can significantly augment separation and mixing. Thus control of pairing may be a method of controlling separation or mixing. While mixing is not necessarily caused only by pairing, pairing following the formation of ribs can cause mixing of core fluid by folding and spanwise stretching (and the consequent possible reconnection within rolls). Pairing affects vortex core deformation, fine-scale mixing and helicity generation, and may involve significant vortex reconnection activity. The pairing contributes to a counter-gradient transport of momentum and is an additional cause for the breakdown of coherent structures (and generation of fine-scale turbulence), as shown by Hussain \& Zaman (1980) in their study of a circular jet near field. Ho \& Huang (1982), among many other researchers, have discussed pairing in a plane mixing layer. Pairing can also be important in aerodynamic noise generation. Laufer (1974) first hypothesized vortex pairing to be a primary source of jet noise, a mechanism that found support from Acton (1977), Ffowcs-Williams \& Kempton (1978) and Crighton (1981). Further, Kibens (1980) suggested that the puzzling phenomenon of broadband noise amplification, found by Moore (1977) and Bechert \& Pfizenmaier (1975) in excited jets, was due to spatial jitter in pairing events. Following vortex sound theory (Möhring 1978; Kambe 1984; Obermeier 1985), which relates the far-field pressure to the third time derivative of the source vorticity moment, it appears that the details of the vorticity field and its spatiotemporal evolution during pairing (which involves rapid acceleration and deceleration of vortical fluids) are crucial in understanding and controlling aerodynamic noise generation.

In this paper, we address the dynamics of coherent structures during their pairing for a limited downstream distance defined by $1 \leq x / \mathrm{D}_{\mathrm{e}} \leq 5$. In this region, the azimuthal curvature of the jet is an important parameter in the instability mechanism of the jet. This is unlike the situation very close to the exit plane where the momentum thickness $\theta$ « $D_{e}$; in this case the instability mode scales with the exit momentum thickness $\theta_{\mathrm{e}}$. In other words, the development of the short waves close to the jet exit plane are insensitive to the jet geometry, while the long waves farther downstream are dependent on the jet dimension (Batchelor \& Gill 1962, p. 538). We differentiate the instabilities in these two regions and designate these as the 'shear layer mode' and the 'jet column mode' instabilities (Zaman \& Hussain 1980; Laufer \& Zhang 1983). Petersen \& Samet (1988) employed linear analysis by taking into account the radial dependence, but found no difference between the shear layer and jet column instability modes. However, there is indeed a difference between the instabilities of short- 
wave instability mode and longer modes whose wavelengths are comparable to the jet diameter (see Bachelor \& Gill 1962).

The instability leading to pairing and the topological details of coherent structures during pairing are not only of fundamental concern in turbulence physics, but also of crucial importance for effective turbulence management (both enhancement and suppression of turbulence phenomena such as transports of heat, mass and momentum, combustion, drag and aerodynamic noise). Additionally, the details of structure evolution for well-defined initial and boundary conditions can serve as target data for validation of modeled and direct numerical simulations.

The instability mechanism leading to pairing (known as subharmonic resonance) was first analyzed by Kelly (1967), who used a weakly nonlinear analysis for a temporally growing shear layer. More recently, Monkewitz (1988) modified the theory for a spatially developing shear layer. His analysis is for a small region near the saturation location of the fundamental, but does not address the saturation of the subharmonic (i.e. occurrence of pairing). Numerically, Patnaik et al. (1976), Acton (1976), Riley \& Metcalfe (1980) and Corcos \& Sherman (1984) have studied temporally-evolving shear layers, while Ashurst (1979), Mansour \& Barr (1985), Grinstein et al. (1987) and Mansour et al. (1988) have explored pairing in spatially developing mixing layers.

No corresponding theoretical study has addressed pairing in the jet column where vortices are fat and their azimuthal curvature is important. An energy-integral technique was used by Mankbadi (1985) to study the interactions between a fundamental and its subharmonic in a circular jet. Raman \& Rice (1989) studied subharmonic resonance in the circular jet column using two-frequency excitation ( $f$ and $f / 2$ ). Similar to what was found in the shear layer domain, they observed dependence of the growth of the subharmonic on its initial phase and on the critical fundamental amplitude. Cohen \& Wygnanski (1987) studied the interaction of two waves of different azimuthal modes in circular jets. They observed the resonant growth of a subharmonic wave whose azimuthal mode number is equal to the difference between the imposed numbers of modes of the fundamental and the subharmonic.

Experimentally, almost everyone studying shear layers and jets has observed pairing (notably Sato 1959; Wille 1963; Browand 1966; Winant \& Browand 1974; Brown \& Roshko 1974). Most experimental investigations of vortex pairing have been based on either flow visualization or spectra of the longitudinal velocity; there have been very few quantitative studies of coherent structure dynamics associated with pairing. This phenomenon has been of considerable interest in our laboratory - - in particular, the pairing dynamics of coherent structures in an axisymmetric jet (Hussain \& Zaman 1980) and the role of pairing in jet noise (Bridges \& Hussain 1987).

In part 1 of this paper (Hussain \& Husain 1989; referenced herein as HH), we have discussed the out-of-plane deformation of elliptic vortices due to self-induction and the parameters which govern pairing in elliptic jets. In this part we focus on the details of the pairing mechanism of nonplanar vortical structures and its effects on various turbulence measures: namely, coherent 
velocities, incoherent turbulence intensities, incoherent and coherent Reynolds stresses, turbulence production, mass entrainment, etc. The dynamics of coherent structure interaction have been examined from the educed coherent vorticity field. Significant effects are highlighted by comparing the results of the elliptic jet with those of a circular jet. We then address details of coherent production of incoherent turbulence and discuss the role of ribs in the production mechanism. Finally, time-average measures, including their modification under stable pairing mode of excitation, are discussed in terms of coherent structure dynamics. Further details were documented by Husain (1984).

\section{Eduction technique}

Velocity data from a flow domain may suggest large-scale organized events, only when the latter are dominant. Large-scale organization, however, can be buried under higher-amplitude, random turbulence; one must find ways to filter out the random field and focus on the underlying organized events. Of course, one must also consider the coupling between the two fields. By coherent structure we mean the ensemble average of properly aligned, organized vortical motions of the same mode (i.e. the same size, shape, strength and orientation) at a particular phase of their evolution. We use phase-average to denote this phase-aligned ensemble average. Phase averages at different phases provide the time evolution of coherent structures. Using such a phase averaging procedure, incoherent turbulence can be separated from the coherent part without any ambiguity, and the topology and dynamics of coherent structures and their coupling with incoherent turbulence can be studied quantitatively (Hussain 1981).

Several approaches have been used to educe coherent structures. An ideal approach would be to obtain three-dimensional vorticity fields as a function of time and apply our eduction scheme to these data. Due to the limitations of the current measurement techniques, studies of structures have been limited so far to detection based on spanwise vorticity in spanwise planes, rather than the full three-dimensional details of flow; also, the measurements unavoidably suffer from limited spatial resolution.

Present method. Pairing in an unexcited jet occurs somewhat randomly in space and time. Eduction of naturally occurring pairing events using a single-point detection scheme (similar to that used by Browand \& Weidman 1976, Bruun 1977, Yule 1978 and Zaman \& Hussain 1984) will suffer from smearing because the eduction scheme cannot differentiate between structures of different sizes, shapes and strengths, and their transverse locations. A multi-probe data acquisition technique with proper conditioning of the measuirement signals reduces smearing (Tso 1983, Hayakawa \& Hussain 1985), but requires the use of Taylor's hypothesis, which introduces substantial error in situations where structures undergo interactions such as pairing or tearing (Zaman 
\& Hussain 1981). Furthermore, a rake is ineffective when structures are small because of the unavoidably coarse transverse spatial resolution (due to X-wire probe separation). To study the pairing dynamics with currently available technology, particularly in the near field of a jet, the small thickness of the shear layer forces the use of phase-locked measurements with a single probe (of course without invoking Taylor's hypothesis).

A periodic perturbation greatly facilitates eduction by temporally stabilizing structure formation and evolutionary stages. This substantially reduces the variance in structure size, shape, orientation and strength, and minimizes smearing in the educed structures. Additionally, the (nearly periodic) structures are detected using a local trigger probe, allowing more accurate alignment of detected events. Further improvement can be achieved by optimizing the alignment through cross-correlation, but is not warranted in the early stages of the evolution.

Inevitably, questions arise concerning the relcvance of excited structures to unexcited ones. Structure formation and pairing in an unexcited jet are induced by uncontrolled ambient disturbances and feedback from downstream structures. Controlled excitation can overshadow these disturbances and remove the associated phase jitter, thus allowing phase-locked measurements with a single sensor. One would expect that such excitation will have significant effects on the time-average measures because of stabilized periodic pairing in an excited jet as opposed to jitter in pairing in an unexcited jet. However, when pairing occurs in an unexcited jet, we expect that the instantaneous coherent structure evolution should not be drastically different form that undergoing pairing in an excited jet. It was argued and later proven that small to moderate periodic excitations can pace the initiation of structures without noticenbly altering the structures (Hussain \& Zaman 1980).

This method offers spatial resolution limited only by the probe size -- particularly desirable in situations where the flow domain is too small for a rake of X-wires. However, we reiterate that while excitation is effective in reducing initiation jitter, it cannot eliminate evolutionary jitter (Hussain 1986). There are, therefore, limitations as to how far downstream the single-sensor technique can be used because of increasing jitter with increasing $x$. Considering spatial resolution and the need for Taylor's hypothesis when a rake is used, the phase-locked measurement technique with a single sensor was considered to be a better compromise for the present study.

\section{Experimental procedure}

The jet facilities (air for hot-wire measurements and water for flow visunlization) used in the present study have been described in HH. The probe configuration and the coordinate system are shown in figure $1(a, b)$. Because the elliptic jet cross-section switches axes, we need to specify reference planes. We use 'major plane' and 'minor plane' to denote the reference planes defined at the nozzle exit -- they are the planes passing through the exit major axis and minor axis respectively. 
In this study we used a $2: 1$ aspect ratio ellipric jet of equivalent diameter $D_{c}=5.08 \mathrm{~cm}$ with an initially laminar boundary layer having a constant momentum thickness all around the nozzle exit perimeter (nozzle $N 1$ in $H H$ ). Equivalent diameter is defined as $D_{c}=2(a b)^{1 / 2}$, where $a$ and $b$ are the semi-axes at the nozzle exit. For the data in the present paper, the jet exit speed was $9.5 \mathrm{~ms}^{-1}$ corresponding to $\mathrm{Re}_{\mathrm{De}}=3.2 \times 10^{4}$. The reason for using the unusual situation of a constant momentum thickness is explained in $\mathrm{HH}$, which also emphasizes the role of initial condition (i.e. jet exit flow) and documents the quality of the flow, including longitudinal mean and ms fluctuation velocity profiles of the exit boundary layer, but at a higher Reynolds number of $\mathrm{Re}_{\mathrm{Dc}_{\mathrm{c}}}=10^{5}$. For the present study, the exit boundary layer characteristics were rechecked in both the major and minor planes of the elliptic cross-section. Boundary layer profiles were laminar, had low fluctuation level and agreed very well with the Blasius profile, having shape factors close to 2.59; the exit momentum thickness $\theta_{\mathrm{e}}$ was $0.23 \mathrm{~mm}$, thus assuming a top-hat profile with elliptic support. Stable pairing was induced via sinusoidal bulk forcing at an exit plane excitation level $\left(u u^{\prime}, \mathrm{U}_{\mathrm{e}}\right)$ of $2.5 \%$ using an acoustically-driven longitudinal resonance of the settling chamber cavity (see $\mathrm{HH}$ ). The excitation frequency and amplitude level, and flow velocity were chosen carefully so that the centerline velocity signals were devoid of any modulations near the location of pairing. Such a requirement is necessary for structure eduction using the present phase-locked measurement technique. Velocity signals started modulating (i.e. detuning) as the excitation amplitude was lowered below about $2 \%$, signifying that the pairing location was not fixed in space.

Flow visualization studies were made in a submerged water jet facility using a laser-induced fluorescent dye (uranamine). Controlled perturbations were introduced by an electromagnetic shaker. The sinusoidal motion of the shaker actuated a piston in a cylinder containing water. The cylinder is connected to the outer perimeter of the shear layer excitation chamber through four tubes (for details, see $\mathrm{HH}$ ). With this excitation system, the amplitude of excitation dropped rapidly at frequencies higher than about $8 \mathrm{~Hz}$ because of the large inertia of the oscillating water column in the excitation system. Thus, in order to obtain $S_{t D c}=0.85$ with a reasonable amplitude, we had to choose a frequency of $5.76 \mathrm{~Hz}$, resulting in a lower $\mathrm{Re}=1.7 \times 10^{4}\left(\mathrm{U}_{\mathrm{e}}=0.344 \mathrm{~ms}^{-1}\right)$ than the $\mathrm{Re}$ used in the air jet.

Response to excitation. HH showed that coherent structures in elliptic jets with moderate aspect ratios are characterized by the jet column mode of stable pairing that occurs at $\mathrm{St}_{\mathrm{De}_{\mathrm{c}}}\left(\equiv \mathrm{fD}_{\mathcal{C}} / \mathrm{U}_{c}\right)=0.85$; here $\mathrm{f}$ is the excitation frequency. As mentioned above, experiments in $\mathrm{HH}$ were made at $\mathrm{Re}_{\mathrm{DC}}=10^{5}$; in the present study, however, coherent structures were educed at a much lower $\operatorname{Re}_{\mathrm{De}}=3.2 \times 10^{4}$ for better spatial resolution because at this lower speed the structures are larger. The jet column mode of stable pairing was again checked at this $\mathrm{Re}_{\mathrm{Dc}}$ and found to occur optimally at $\mathrm{St}_{\mathrm{Dc}}=0.85$, supporting our clain that this mode is fairly independent of $R_{\mathrm{De}}$ (at least within the $\mathrm{Re}_{\mathrm{De}}$ range we have studied). 
Figure 2(a) shows the evolution of the jet centerline $u$-spectrum under excitation at $\mathrm{St}_{\mathrm{De}}=0.85$; all the traces have the same logarithmic ordinate and linear abscissa scales. The spectra, averaged over 128 realizations with a frequency resolution of $0.3 \%$ of the maximum, were obtained with a real-time spectrum analyzer (Spectrascope SD335). As the streamwise distance increases, a subharmonic component (associated with the first pairing) appears, overtakes the fundamental at $x / \mathrm{D}_{\mathrm{e}} \cong 0.5$, and grows to a maximum at $x / \mathrm{D}_{\mathrm{c}} \cong 2.0$. Note that the subharmonic (associated with the larger, paired structures) persists longer than the fundamental, as to be expected. Due to nonlinear interactions, higher harmonics of the subharmonic and of the fundamental first grow and then decay until they become submerged in the evolving background turbulence (for $x / D_{e}>6$ ); beyond this length the spectrum is fully developed and devoid of any noticeable peak. This is by no means an indication that turbulence is fine-grained beyond $x / \mathrm{D}_{\mathrm{c}} \cong 6$; organized structures are in a state of largescale breakdown, they lose their phase coherence and their random phase leaves no discernible spectral footprint. There is no second subharmonic ( $f / 4)$ component, suggesting that only one pairing occurs before breakdown.

Since coherent structures were to be educed by phase-locked measurement, the centerline longitudinal velocity signal $u_{\mathrm{c}}(t)$, which was used as the trigger signal, was examined regarding its variation in amplitude, jitter in time and superimposed fine-scale features. Figure 2(b) shows one such set of $u_{\mathrm{c}}(\mathrm{t})$ as a function of $x$. The traces also show the gradual development of the subharmonic frequency followed by its saturation, harmonic growth and transition to turbulence. Note that at $x / D_{c}=3.25$, there is laminar flow on the jet centerline between successive turbulent vortical structures. The signal is precisely sinusoidal at the exit but takes a cusp-like shape (around $x / D_{\mathrm{e}} \cong 2$ ) after the frequency is halved. This corresponds to the situation where there is intense velocity oscillation on the jet centerline associated with localized periodic pairing. The velocity signal shows a decrease in amplitude from the exit value to a minimum (around $x / \mathrm{D}_{\mathrm{e}} \cong 0.25$ ) before its growth mostly due to the subharmonic. Such suppression has been observed also in our excited circular jet studies and explained previously.

The periodic signal up to $x / \mathrm{D}_{\mathrm{e}}=2.5$ (also evident from the sharp spectral peak) indicates periodic passage of structures of equal strengths. This is important for phase-locked measurement because this technique assumes that all the structures are of the same kind, although exact periodicity of the signal is not expected.

Phase averaging. Eduction involves the separation of coherent and incoherent components $\langle g\rangle$ and $g_{\mathrm{r}}$ of any field quantity $g(x, t)$ obtained by phase-averaging a field variable $g$ at a particular phase. The coherent part is the ensemble average of the phase-aligned events at a particular phase (i.e. phase average). Interpretation of the decomposition of coherent and incoherent fields (double and triple decompositions) has been discussed at length previously (Hussain 1981). Most of the important coherent structure properties (e.g. coherent vorticity, coherent turbulence production, 
incoherent normal and shear stresses) are independent of the choice of a steady reference frame. However, streamlines and velocity vector patterns are not Galilean invariant, and require careful interpretation (discussed further in $\$ 4.1$ ).

Time- and phase-average field measurements were made in both the major and minor planes. Data presented in this paper cover spatial distributions of the following time- and phase-average quantities: mean velocities $\mathrm{U}$ and $\mathrm{V}$, turbulence intensities $u^{\prime}$ and $v^{\prime}$; Reynolds stress $\overline{u v}$; timeaverage shear, normal and total productions $\mathrm{P}_{s}, \mathrm{P}_{n}$ and $\mathrm{P}$; phase-average velocities $\langle u\rangle$ and $\langle v\rangle$; spanwise coherent vorticity $\left\langle\omega_{\mathrm{z}}\right\rangle$; incoherent turbulence intensities $\left\langle u_{\mathrm{r}}{ }^{2}\right\rangle^{1 / 2}$ and $\left\langle v_{\mathrm{r}}{ }^{2}\right\rangle^{1 / 2}$; incoherent and coherent Reynolds stresses $\left\langle u_{\mathrm{r}} v_{\mathrm{r}}\right\rangle$ and $\left\langle u_{\mathrm{p}} v_{\mathrm{p}}\right\rangle$; and coherent shear, normal and total productions $\left\langle\mathrm{P}_{\mathrm{s}}\right\rangle,\left\langle\mathrm{P}_{\mathrm{n}}\right\rangle$ and $\langle\mathrm{P}\rangle$. The significance of these measures relating to coherent structure dynamics was illustrated previously (Hussain 1981, 1983).

Data acquisition. For phase-average measures, the reference probe was located on the jet centerline at $x / \mathrm{D}_{\mathrm{e}}=1.8$ where the $\mathrm{f} / 2$ component of the $u$-signal was the strongest, minimizing the effect of jitter on the educed structures. The $\|$-signal there was bandpass-filtered at $f / 2$ and hen used as the reference signal to the triggering device. The output signal from the triggering device flagged the computer (when the signal crossed a threshold) to sample data from an $X$-wire at that instant. The adjustable dwell time of the triggering circuit allowed the delay time $t$ (between the instants of structure detection and signal sampling) to be set such that the structure would be captured (i.e. educed) at the desired phase.

The phases for eduction were selected after examining the flow by visualization. We noted that complete merger of vortices occurred in the major plane, but no merger took place in the minor plane. To examine the details, one full cycle of the pairing process in the minor plane was divided into five phases (I to $\mathrm{V}$ ). The structures were also educed in the major plane at exactly the same phases.

At each phase, measurements were carried out at a large number of spatial points in both major and minor planes. The number of grids and their spacing were judiciously varied in the streamwise and transverse directions with a finer spacing in the vortex core region. Thirty grids were used in the streamwise direction, while the number of grids in the transverse direction varied between 20 to 30. For a given location $(x, y)$ of the $X$-wire, an average over a large ensemble (typically 2000 to 3000 samples, which gave convergent $\langle u\rangle$ and $\langle v\rangle$ values within $5 \%$ scatter) of data obtained at successive trigger points (i.e. at identical locations of successive structures) gave the phase-average at that point. This process was repeated after relocating the measurement probe, but keeping the trigger conditions unchanged. Results are presented as contour plots. Positive-valued contours are denoted by solid lines and negative-valued ones by dashed lines. 


\section{Results and discussion}

\subsection{Coherent vorticity}

Figure 3(a) shows contours of coherent azimuthal vorticity $\left\langle\omega_{z}\right\rangle$, nondimensionalized by the excitation frequency $\mathrm{f}$; the five sequential phases ( $\mathrm{l}$ to $\mathrm{V}$ ) during the pairing process are shown in both major and minor planes. The trigger times were chosen so that pairing of vortices are captured at different relative inclinations. For example, the line connecting the two vortices in the minor plane would be inclined at an angle $\theta \cong 45^{\circ}$ with the $x$-axis at phase I. At phases II to V, these angles were to be approximately $90^{\circ}, 120^{\circ}, 127^{\circ}$ and $155^{\circ}$ respectively. These angles could not be preselected exactly, as they were apparent only after eduction. In the following discussion, we will refer to coherent vorticity simply by vorticity.

To compare pairing in elliptic and circular jets, coherent structures in a circular jet were also educed under the same conditions (e.g. same nozzle exit area, exit velocity, excitation frequency, level of excitation and phases) using the same facility, but with the elliptic nozzle replaced by a circular nozzle. Due care was taken to check the initial (laminar boundary layer) condition (mean and fluctuation level profiles) and axisymmetry of the flow. Structures were educed at three phases for $\theta$ values of $50^{\circ}, 90^{\circ}$, and $130^{\circ}$. These three phases correspond approximately to phases I, II and IV of the elliptic jet. Contours of $\left\langle\omega_{\mathrm{z}}>/\right.$ f for the circular jet are shown in figure $3(b)$.

\subsubsection{Vorticity contours.}

In circular jets, self-induction is constant all around the perimeter and pairing occurs nearly uniformly around the perimeter (Hussain \& Zaman 1980). In an elliptic jet, however, interaction is quite different in the major and minor planes. Merger of vortices occurs in the major plane (phase III), while in the minor plane, the trailing vortex, instead of pairing, rushes through the leading one by mutual induction and subsequently breaks down. Contours of $\left\langle\omega_{z}\right\rangle$ in the minor plane show the distinct identities of the two vortices even up to phase $V$. A third region of vorticity concentration, seen in the upper right-hand corner at phases IV and $V$ (in the minor plane), is the remnant of the leading vortex of the previous (downstream) vortex pair. There is no remnant of the trailing vortex of the previous pair as it has already advected beyond the measurement region. The broken-down structure farther downstream, if captured using the present mensurement technique, would suffer from significant smearing; therefore the structures were not educed farther downstream.

The low level vorticity contour $\left.\left(<\omega_{2}\right\rangle / \mathrm{f}=0.5\right)$ in the major plane shows a valley on the lowspeed side (figure 3a). Due to self-induction, the vortex cores in the major plane move toward the jet axis shedding low vorticity fluid in their wakes on the low-speed side; this is what produces the valley. In this region the ambient potential fluid is engulfed and subsequently imparted vorticity by diffusion (compare phases IV and V). This region, where the vorticity has a local minimum, is shown as a hatched area. Such discard of turbulent vortical fluid in the wake of vortical structures is 
not unlike that observed by Maxworthy (1974). Note that all the vorticity contours show a mild dip on the low-speed side of the minor plane. This is a result of the combined effects of flow reversal and the arrival jitter of the leading vortex in the measurement region. Because of the inability of the hot-wire to detect flow reversal, as well as due to increased jitter, the measurements on the lowspeed side of the outer structure are contaminated (explained by Hussain \& Zaman 1980).

\subsubsection{Peak vorticity}

The evolution of vorticity peaks during the interaction process is shown in figure $4(a, b)$ for both elliptic and circular jets. Since we have educed structures in the circular jet for only three phases, the peak vorticity curve is extended up to $x / D=4$ using data from Hussain \& Zaman (1980). In the minor plane, the vorticity of the leading structure diffuses more slowly than that of the trailing vortex. Between phases $I$ and $V$, the peak vorticity $\left\langle\omega_{z}\right\rangle_{p}$ of the leading structure is reduced from 6 to 4 , while that of the trailing structure is reduced quite significantly -- from 10 to 2 . Between phases I and III, as the trailing vortex rushes inside the leading one, the former's $\left\langle\omega_{2}\right\rangle_{p}$ is reduced at a high rate as its perimeter is decreased. [This decrease in the perimeter length may not be simple as azimuthal wrinkles are likely to occur.] Later, $\langle\omega\rangle_{\mathrm{p}}$ is further reduced by rapid diffusion because of turbulent breakdown of the trailing vortex.

The elliptic vortices undergo deformation due to self-induction, and the leading vortex switches axes prior to the pairing interaction. Such deformation increases vorticity due to stretching; consequently, the diffusion of vorticity, mostly due to incoherent turbulence (see $\S .4 .3$ ), is also increased. At phase II, the circumferential length of the leading vortex is increased by about $30 \%$ (based on the local perimeter), indicating that the leading vortex has undergone substantial stretching. Measurements show that in the minor plane, the leading vortex has higher levels of $\left\langle u_{r}^{2}\right\rangle 1 / 2$ and $\left\langle v_{\mathrm{r}}^{2}\right\rangle 1 / 2$ than the trailing vortex (discussed in $\S 4.3$ ). Thus an increase in peak coherent vorticity due to stretching balances an opposing decrease due to turbulent diffusion, keeping the peak vorticity nearly constant up to phase IV. In the major plane, the decrease in peak vorticity is less dramatic. Since in this plane vortices converge toward the jet axis, there is no stretching. It appears that a balance between an increase in coherent vorticity due to merger of vortices and a decrease due to turbulent diffusion prevents a large decrease in the peak vorticity up to phase IV.

On the major-axis side, pairing does not occur through the leapfrog motion of planar vortices found in a circular jet. Instead, merger occurs over a short length on the major axis sides through an entanglement process (discussed in $\S 4.1 .4$ and $\S 4.1 .5$ ). A comparison between structure contours in the circular and elliptic jets is meaningful only in the minor plane, because the interactions in this plane up to phase IV appear to be similar. In the minor plane, $\left\langle\omega_{z}\right\rangle_{p}$ of the trailing vortex is decreased at a much higher rate than in the circular jet, while $\left\langle\omega_{z}\right\rangle_{p}$ of the leading vortex is almost the same up to phase III as that in the circular jet. Farther downstream, structures in the circular jet 
undergo pairing, while the trailing vortex in the elliptic jet breaks down, resulting in a large decrease in peak vorticity.

\subsubsection{Vorticity diffusion and mixing.}

Detailed mechanisms of mass entrainment and fine-scale mixing are quite complex and their direct measurements are difficult. Integral measures, such as increase in jet width, mass flux and domains of high level of turbulence intensity, have been used to estimate mass entrainment and mixing in the time-mean sense. Educed coherent structures allow a closer examination of mechanisms of mass entrainment and vorticity diffusion, and provide an estimate of the amount of mixing which occurs during their evolution. Since we studied the elliptic and circular jets under identical initial conditions and using the same detection scheme, we can compare the effects of geometry modification alone on various structure properties. Here we compare the areas enclosed by various levels of vorticity contours for elliptic and circular jets. Such areas (ranging from $<\omega_{z}>/ f=1$ to the maximum level at each phase) in the major and minor planes of the elliptic jet are shown in figure $5(\mathrm{a}, \mathrm{b})$ for the five phases. The corresponding circular jet data are shown in figure $5(\mathrm{c})$ for phases I, II and IV. In the circular jet, areas enclosed by a particular vorticity contour level show very little change from phases I to IV. In the elliptic jet, the decrease in $\left\langle\omega_{Z}\right\rangle_{p}$ and in the areas enclosed by $<\omega_{Z}>/ f>1.5$ contours, and an increase in the areas for $\left\langle\omega_{\mathrm{Z}}>/ \mathrm{f}<1.0\right.$ are quite significant. Note that both the decrease in the peak value of $\left\langle\omega_{z}\right\rangle$ and the increase in the $\left\langle\omega_{z}>/ f=1\right.$ contour area are higher (about twofold) in the minor plane.

Variations of area enclosed by the vorticity level $\left\langle\omega_{z}>/ f=1\right.$ are shown in figure 5(d). At each phase, the $x / \mathrm{D}$ location denotes the axial distance of the mid-point of the two interacting structures from the jet exit plane. For comparison, circular jet data from Hussain \& Zaman (1980) are also included, which show good agreement with the present circular jet data, establishing the reliability of the two independent measurements. These curves show clearly that the structure boundary defined by a low level vorticity contour $\left.\left(e . g .<\omega_{z}\right\rangle / \mathrm{f}=1\right)$ increases at a much higher rate in the elliptic jet than in the circular jet, especially during phases I to III. Note that this area in the minor plane is higher by about $20 \%$ at phase I and $60 \%$ at phase IV than in the circular jet. This indicates that more (nonvortical) ambient fluid is engulfed in the elliptic jet than in the circular jet; that is, mixing in elliptic jets is more rapid than in circular jets. The jet cross-section of elliptic and circular jets reported by HH are consistent with these observations.

\subsubsection{Vortex trajectories.}

The trajectories of the vortex pairs during interaction are shown in figure 6(a). Solid lines are derived from the locations of peak $\left\langle\omega_{z}\right\rangle$ in figure 3(a). Dashed lines (nearer to the exit plane) are inferred from the $U / U_{c} \cong 0.5$ line. Dotted lines denote trajectories for the circular jet. 
The lip lines (i.e. constant radius lines passing through the nozzle lip) along the major and minor planes for the nozzle used in this study $\left(2: 1\right.$ aspect ratio and $\left.\mathrm{D}_{\mathrm{e}}=5 \mathrm{~cm}\right)$ are $0.707 \mathrm{D}_{\mathrm{e}}$ and $0.353 \mathrm{D}_{\mathrm{e}}$ away from the jet centerline (these are the values of semi-axes $\mathrm{a}$ and $\mathrm{b}$ ). In the major plane, the transverse locations of both vortex centers (i.e. locations of peak vorticity) at phase I are equal, being approximately $0.6 \mathrm{D}_{\mathrm{e}}$; the vortices in the major plane have moved transversely toward the jet axis by self-induction. In the minor plane, the transverse distances are approximately $0.65 \mathrm{D}_{\mathrm{e}}$ and $0.35 \mathrm{D}_{\mathrm{e}}$ for the leading and trailing vortices respectively, indicating that the leading vortex has moved a considerable distance away from the jet axis. A comparison of transverse locations of vortex centers in both planes at phase I shows that the leading vortex has already switched axes, while the trailing vortex aspect ratio has decreased only slightly.

In the minor plane, the leading vortex moves away from the jet axis rapidly, while the trailing vortex moves almost parallel to the jet axis. That is, the distance between the two neighboring vortices increases rapidly and monotonically, unlike in the circular jet. Note that at phase IV, the

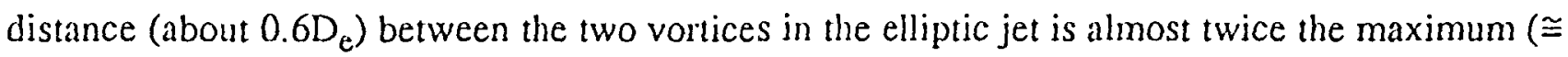
$0.025 \mathrm{D}$ ) between the vortices in the circular jet (see figures $3(a, b)$ ). It appears that this larger distance between the two interacting vortices prevents them from pairing in the minor plane. In the major plane, however, both vortices move toward the jet axis by self-induction; as the two vortices get close they undergo pairing by mutual induction at $x / D_{c} \cong 2.2$.

To explain the differences between vortex interaction in the major and minor planes, let us consider the motions of two adjacent elliptic vortices due to self- and mutual inductions. In figure $6(b, c)$, the directions of self- and mutual inductions are denoted qualitatively by vectors " $S$ " and " $M$ " respectively. In the minor plane (figure 6b), since the resultant of both "S" and " $\mathrm{M}$ " of the leading vortex is in a direction away from the jet axis, the leading vortex moves away from the trailing vortex (thus increasing the distance between the two). This large separation prohibits merger in this plane. In the major plane (figure $6 \mathrm{c}$ ), the leading vortex has already moved toward the jet axis by selfinduction before the trailing vortex is formed. After the formation of the trailing vortex, the resultant of "S" and " $M$ " pushes it to move toward the jet axis, and the two vortices get closer, allowing merger to occur. Note that complete merger in the major plane occurs within the short distance of about $x / D_{e}=0.2$ (see phases II and III of figure 3a), while leapfrog motion in the circular jet continues over a distance of about $x / \mathrm{D}=2$ before the completion of merger.

\subsubsection{Flow visualization.}

The pairing mechanism was further studied by flow visualization. A sheet of laser light was used to examine cross-sections of the structures in both the major and minor planes (see HH for details of visualization technique). Digitally enhanced video frames are shown in figure 7(a) in the form of isointensity contours of fluorescent dye. In this figure, the high speed side of the shear layer 
is below it. These pictures support the interaction process revealed by the measured vorticity contours: complete merger of vortices in the major plane, but no such merger in the minor plane.

In the minor plane, as the interaction proceeds, the trailing vortex rushes inside the leading one and breaks down violently soon after it emerges downstream. In frames 6 to 8, isolated, fragmented contours show the broken-down parts of the trailing vortex. This process of violent breakdown appears to enhance small-scale mixing in elliptic jets. In reference to our longstanding warning about the limitations of flow visualization in understanding structure dynamics in turbulent flows, a question obviously arises as to whether these isointensity contours, showing dye concentrations, represent vorticity concentrations. Since these events occur near the dye injection location (between 2 and $3 \mathrm{D}_{\mathrm{c}}$ ) and within a short travel time, the concentrations of vorticity and dye cannot be distinctly different; of course, farther downstream, the correspondence weakens even at unity Schmidt number because of 3D motions.

Although the vorticity contours in the major plane exhibit merger, similar to that exhibited in a circular jet, the pairing process in an elliptic jet is morphologically different from that in a circular jet. This difference was revealed by flow visualization using flood lighting, which enabled us to observe the interaction process simultaneously over the entire perimeter. In a circular jet the pairing interaction takes place through a leapfrog motion of two vortices along the entire perimeter wherein the vortex lines remain locally parallel (see also Reynolds \& Bouchard 1981). In an elliptic jet, the two neighboring vortices come close to ench other orily in the major plane and undergo pairing along a small segment there. This starts as an entanglement rather than leapfrogging, although the entangled vortices soon become a single vortex through diffusion. As an aid to the description of pairing, inferred from data (figure 3a) and observed through visualization, our perception of the pairing process is shown schematically in figure 7(b). A sequence of flow visualization pictures, taken simultaneously from the major- and minor-axis sides, is shown in figure 7(c). For clarity, perspective views are also drawn schematically at the bottom of the flow visualization pictures. These visualization pictures, though they clearly show only local merger, unfortunately cannot reveal the entanglement process.

Flow visualization studies in plane and circular jets have also revealed similar mergers of vortices through entanglement, shown schematically in figure 7(d, e). Merger through entanglement was also observed by Clark \& Kit (1980) in plane jets. It appears that nonuniformity of initial conditions or spanwise perturbations produce local curvature in the vortices of a plane jet, leading to entanglement. In a circular jet, we have observed axisymmetric vortices being tilted alternately forward and backward, presumably by a spiral instability mode, resulting in the entanglement interaction over small sectors. Merger through entanglement may indeed be a common phenomenon in many situations (see also Chandrasuda et al. 1978); elliptic jets can clearly allow us to examine this mode of vortex merger in detail. 


\subsection{Phase-average velocities.}

Measured property contours for the five selected phases are voluminous; in order to save space we discuss hereafter data contours only for phases I, II and IV. In all the figures, vortex centers are identified by a " + " for spatial reference. In the following, we present data primarily for documentation, and comment on them whenever possible.

\subsubsection{Longitudinal and transverse velocities.}

Phase-average longitudinal and transverse velocity contours $\langle u\rangle / U_{\mathrm{c}}$ and $\langle v\rangle /$ Ue are shown in figures 8 and 9 respectively. The distributions of $\langle u\rangle$ and $\langle v\rangle$ can be qualitatively explained by decomposing the flow field into motions produced by self-induction (curvature-dependent induction) and mutual-induction (by all other vortices), and by the rotational flow field of the vortex itself, which we will call 'induced' and 'rotational' motions.

Contours of $\langle u\rangle$ show only positive regions, because the structures advect in the streamwise direction at a speed higher than the opposing motion due to rotation on the low speed side. Obviously, a hot-wire cannot differentiate the direction of $\langle u\rangle$, but if flow reversal occurred, contours of $\langle u\rangle$ would show a peak region on the low-speed side. Near the jet axis, all motions are in the flow direction, producing a peak in $\langle u\rangle$ distributions which is higher than the exit velocity. The 'S-shaped' contour lines in phase II in the minor plane are due to the opposing rotational motion between the structures --- $\langle u(y)>$ decreases away from the imner vortex and then increases near the core region of the outer vortex.

Each vortex is associated with a positive $\langle v>$ region at the front (downstream) and a negative region at the back (upstream) of its center, primarily due to rotational motion. However, the transverse motions of the vortices due to self- and mutual inductions complicate the $\langle v>$ contours. Consider the $\langle v\rangle$ contours at phases I and II in the major plane. Between the structures, the transverse velocities induced by the two vortices nearly cancel each other, causing a considerable decrease in the regions of both positive and negative $\langle v\rangle$ and their corresponding peak values. In phase II, the inward motion of the trailing vortex (due to self- and mutual inductions) is strong enough to overshadow the outward fluid motion at its front (between the pair), producing a region of only negative $\langle v\rangle$.

In both planes, $\langle v\rangle$ contours show that outward motion at the front is stronger than the inward motion at the back. Both positive and negative peaks of $\langle v\rangle$ contours are higher in the minor plane than in the major plane during initial stages of pairing (phases I-III), but during phases IV and V, these peak values are higher in the major plane. The areas encompassed by a constant negative $\langle v\rangle$ contour level, say $\langle v\rangle=-0.02$, at each phase (except for phase $V$ ) are larger in the minor plane than in the major plane. This indicates that during the initial stages of interaction, the minor-axis side 
is more active in drawing ambient fluid. In this plane, as the trailing vortex rushes through the leading vortex, it pulls in a large amount of ambient fluid. In the major plane, the larger area encompassed by a constant positive contour level, say $\langle v\rangle=0.02$, indicates that a greater amount of jet fluid is ejected in this plane than in the minor plane. It appears that these two different kinds of interactions in the two planes associated with 3D motions produce better mixing in elliptic jets than in circular jets. If one compares the two planes at phase IV, it is clear that motion induced by the vortices is indeed highly 3D and defy simple explanation.

\subsubsection{Convection velocity.}

Convection velocity $v_{c}$ of advecting structures, computed from $\langle u\rangle$ and $\langle v\rangle$ values corresponding to the vortex centers, is shown in figure 10. For comparison, $v_{\mathrm{c}} / \mathrm{U}_{\mathrm{e}}$ distributions for the circular jet (reproduced from Zaman \& Hussain 1980) are also included in this figure. Note that $\left\langle u>\right.$ is an order of magnitude higher than $\langle v\rangle$ at the vortex center (figures $8 \& 9$ ), indicating that $v_{c}$ is mostly longitudinal.

In the major plane, where vortices undergo pairing, the difference in $v_{\mathrm{c}}$ of the leading and trailing vortices is much less than that in the circular jet. Clearly, pairing through entanglement does not allow a large difference in $v_{\mathrm{c}}$. In contrast, in the circular jet, the vortices undergo a 'leapfrog'

motion that involves a large increase in $v_{\mathrm{c}}$ of the trailing vortex and a smaller decrease in $v_{\mathrm{c}}$ of the leading vortex and then a reversal in $v_{\mathrm{c}}$ before merger.

In the minor plane, the trailing vortex rushing inside the leading one has a much higher $v_{c}$ than that of the latter, somewhat similar to, but less than, that in the circular jet. Since the distance between the vortices in the minor plane at each phase $\left(e . g .0 .4 \mathrm{D}_{\mathrm{e}}\right.$ at phase II) is greater than that in the circular jet (e.g. 0.25D at phase II), the mutual induction is less effective in the elliptic jet. This is why the $v_{\mathrm{c}}$ difference is smaller. It is worth noting that the merged vortex (in the major plane) moves faster than in the circular jet by about $10-15 \%$. This is not surprising because the mutual induction across the jet axis is higher in the elliptic jet. In the minor plane, the trailing vortex continues to move faster than shear layer midplane velocity because of self-induction caused by higher curvature (figure $7 \mathrm{~b}$ ).

\subsubsection{Velocity vector.}

Unlike vorticity fields, velocity vector fields are not Galilean invariant, and therefore depend on the choice of the reference frame. To represent vector fields in a meaningful manner, a proper choice of the reference frame is necessary. For an isolated (i.e. noninteracting) vortex, a reference frame moving with the vortex center is the proper choice. However, in a situation where vortices undergo pairing, the choice of a single reference frame is problematic because the interacting vortices advect at different speeds (see figure 10). More complicated is the interpretation of the vector patterns. Of 
course, the flow details associated with one structure can be accentuated by using its center as the reference frame. This will typically obliterate the motion associated with other structures.

In the present study, various reference frames were tried: for example, that of the leading vortex, that of the trailing vortex, a frame moving at the average of the convection velocities of the two vortices, as well as a frame having the velocity halfway between the two vortex centers. No choice is perfect. Not surprisingly, the most satisfactory results were obtained when the midpoint velocity was used as the reference frame velocity. Phase-averaged velocity vectors using this reference frame velocity are shown in figure 11 for the first four phases. In each figure, the vector directions are denoted by arrows and the magnitudes by the line lengths. The corresponding vorticity contours $\left(\left\langle\omega_{\mathcal{L}}\right\rangle / \mathrm{f}=1,3\right.$ and 5 ) are superimposed as dashed lines.

A major advantage of the velocity vector field representation is that it gives a better physical perception of the instantaneous flow field than vorticity contours. It shows relative movements of different parts of a structure or of interacting structures as well as engulfment and ejection of fluids.

Since the advection speeds of the structures in the transverse direction are very small compared to those in the streamwise direction, the transverse components of the velocity vectors correctly depict the engulfment of ambient fluid at the back and ejection of jet core fluid at the front of the structures. A few of these velocity vector plots clearly show the saddle regions (marked X). The saddles between the interacting structures at phases III and IV in the minor plane are not clear because a single reference velocity cannot depict the flow field when the two structures are advecting at quite different velocities. The existence of longitudinal vortices (ribs) in the saddle regions, and their roles in turbulence production and entrainment have been discussed previously (Hussain 1984, 1986).

\subsection{Incoherent Turbulence Stresses and Coherent Production}

The mechanisms of turbulence production and small-scale mixing are central to turbulence physics, but are poorly understood. The coherent structure approach to turbulence has provided us some understanding of the turbulence production mechanism. In this section we will examine the incoherent normal and shear stresses, their contributions to coherent production and the associated mechanisms. In the present study, since we have examined the properties in a plane only, the productions due to incoherent normal stresses $\left\langle u_{\mathrm{r}}{ }^{2}\right\rangle$ and $\left\langle v_{\mathrm{r}}{ }^{2}\right\rangle$, and incoherent Reynolds stress $\left\langle u_{\mathrm{r}} v_{\mathrm{r}}\right\rangle$ are examined.

\subsubsection{Incoherent normal stresses.}

Figures $12 \& 13$ show contours of the phase-average incoherent turbulence intensities $<u_{\mathrm{r}}{ }^{2}>1 / 2 / \mathrm{U}_{\mathrm{e}}$ and $\left\langle v_{\mathrm{r}}{ }^{2}>1 / 2 / \mathrm{U}_{\mathrm{e}}\right.$. The distributions of these two are similar. At all the phases the peaks of incoherent turbulence occur near the coherent structure center. However, there are some 
differences regarding contour boundary and levels. For example, up to phase III, $\left\langle u_{\mathrm{T}}^{2}\right\rangle^{1 / 2} / \mathrm{U}_{\mathrm{e}}$ has a higher peak value in the major plane than in the minor plane, while the higher peak value of $\left\langle v_{\mathrm{r}}^{2}\right\rangle 1 / 2 / \mathrm{U}_{\mathrm{e}}$ occurs in the minor plane. However, peak values of $\left\langle\mathrm{e}_{\mathrm{r}}\right\rangle=\left(\left\langle u_{\mathrm{r}}{ }^{2}\right\rangle+\left\langle v_{\mathrm{r}}{ }^{2}\right\rangle\right)$ in both planes are nearly equal (not shown). For example, at phase II, the peak values of $\left\langle\mathrm{e}_{\mathrm{r}}\right\rangle / \mathrm{U}_{\mathrm{e}}{ }^{2}$ are approximately 0.057 and 0.054 in the major and minor planes respectively. In the circular jet, the peak value of $\left\langle v_{\mathrm{r}}^{2}\right\rangle 1 / 2$ is higher than that of $\left\langle u_{\mathrm{r}}^{2}\right\rangle 1 /$; this is similar to that in the minor plane of the elliptic jet where the structures undergo leapfrog motion (during the first three phases) as in the circular jet.

It is now well recognized that large-scale spanwise coherent structures in shear flows are connected by a spanwise array of nearly longitudinal vortices of alternating signs, known as ribs (Hussain 1984; Bernal \& Roshko 1986; Jimenez et al. 1985). The interactions of ribs and spanwise coherent structures (rolls) play a crucial role in the production of incoherent turbulence. Turbulence produced by the stretching of ribs is advected toward the center of the rolls, resulting in peak values of incoherent turbulence intensities near the roll center. Incoherent turbulence intensity data in jets (Hussain \& Zaman 1980) and wakes (Cantwell \& Coles 1983) show that $\left\langle v_{\mathrm{r}}{ }^{2}\right\rangle{ }^{1 / 2}$ peaks are higher than $\left\langle u_{\mathrm{r}}^{2}\right\rangle 1 / 2$ peaks after the formations of structures; peak values of $\left\langle u_{\mathrm{r}}^{2}\right\rangle 1 / 2$ increase in $x$ and finally exceeds $\left\langle v_{\mathrm{r}}^{2}\right\rangle 1 / 2$ peak values. These data suggest that during the initial stages of vortex rollup and pairing, the rib-roll interactions produce turbulence predominantly in the transverse direction; $\left\langle u_{\mathrm{r}}^{2}\right\rangle 1 / 2$ is produced at a later stage. A higher peak in $\left\langle u_{\mathrm{r}}{ }^{2}\right\rangle 1 / 2$ in the major plane, even at phase I, suggests that transition occurred earlier in the this plane. The role of ribs in turbulence production mechanism is discussed further in section 4.3.3.

Incoherent turbulence can arise from two sources other than ribs. The first is jitter; variations in structure shape, size, orientation and strength may cause improper alignment of structures. However, the present study of near-field structures under excitation should be free from any significant jitter. The second cause can be the fine-scale turbulence superimposed on the spanwise rolls. In the transitional region, one would expect that this contribution to incoherent turbulence is much smaller than that of the ribs.

While interaction of ribs and rolls of orthogonal vorticities produces fine-scale 3-D turbulence, neither the details of their interaction, nor the topology of the rib-roll connection, nor the winding of the rib within the roll is yet understood. This area is the focus of further research in our laboratory.

\subsubsection{Incoherent and coherent Reynolds stresses.}

The contours of incoherent Reynolds stress $\left\langle u_{\mathrm{r}} v_{\mathrm{r}}\right\rangle / \mathrm{U}_{\mathrm{c}}{ }^{2}$ (figure 14) show that the peak value occurs near the saddles of $\left\langle\omega_{\mathrm{z}}\right\rangle$ contours. Similar spatial correspondence between the peaks of $\left\langle\omega_{\mathrm{L}}\right\rangle$ and $\left\langle u_{\mathrm{r}} v_{\mathrm{r}}\right\rangle$ has been observed in other flow situations, namely circular jets (Hussain \& Zaman 1980), wakes (Cantwell \& Coles 1983; Hayakawa \& Hussain 1985) and mixing layers (Metcalfe et al. 1987). We believe that $\left\langle u_{\mathrm{r}} v_{\mathrm{r}}\right\rangle$ is predominantly controlled by the ribs and their 
interaction with the rolls. By refining the eduction scheme, incoherent turbulence arising from jitter effects and its contribution to $\left\langle u_{\mathrm{r}} v_{\mathrm{r}}\right\rangle$ can be reduced significantly. The contribution of superimposed fine-scale turbulence to $\left\langle u_{r} v_{f}\right\rangle$ should be zero. Thus the major generator of incoherent Reynolds stress is the ribs.

Ribs can be viewed as substructures which are characterized by phase correlated vorticity fields normal to the rolls. Since the connections of ribs to rolls occur randomly in spanwise direction, the associated smaller-scale velocity fluctuations appear incoherent with respect to the educed rolls. Spatial correspondence between $\left\langle u_{\mathrm{r}} v_{\mathrm{r}}\right\rangle$ (both positive and negative) and coherent vorticity $\left\langle\omega_{\mathrm{Z}}\right\rangle$ are direct consequences of the topology of ribs and rolls and their interactions. The orientation of ribs, both in the saddle region and the region where they connect the rolls, determines the domains of positive and negative $\left\langle u_{\mathrm{r}} v_{\mathrm{p}}\right\rangle$.

Velocity fields associated with ribs oriented at angles $\alpha<90^{\circ}$ and $\alpha>90^{\circ}$ to $x$ are shown schematically in figure 15(a). For $\alpha<90^{\circ}$, the rotational velocity around a rib will contribute either $(+u,-v)$ or $(-u,+v)$ in a spanwise measurement plane. In either case the value of $\left\langle u_{\mathrm{r}} v_{\mathrm{r}}\right\rangle$ will be negative. When $\alpha>90^{\circ}$, contributions are either $(+u,+v)$ or $(-u,-v)$, giving rise to a positive value of $\left\langle u_{\mathrm{r}} v_{\mathrm{r}}\right\rangle$. The dependence of the sign of $\left\langle u_{\mathrm{r}} v_{\mathrm{r}}\right\rangle$ distribution on the orientation of ribs is shown schematically in figure $15(b, c)$ for a single vortex (e.g. phase IV in the major plane) or for pairing vortices (e.g. phase II in the minor plane). This simple explanation is in qualitative agreement with the measured positive and negative $\left\langle u_{\mathrm{r}} v_{\mathrm{r}}\right\rangle$ distributions.

The peak value of $\left\langle u_{\mathrm{r}} v_{\mathrm{r}}\right\rangle$ is higher in the major plane than in the minor plane. Because of stronger self-induction in the major plane, the distances between the leading and trailing vortices in this plane at phases I and II are longer by about $40 \%$ and $17 \%$ respectively than those in the minor plane. That is, the ribs in the major plane undergo higher stretching, producing higher peak values of $\left\langle u_{r} v_{p}\right\rangle$. After the completion of pairing in the major plane (phase IV), $\left\langle u_{r} v_{r}\right\rangle$ distributions show larger regions of positive $\left\langle u_{\mathrm{r}} v_{\mathrm{r}}\right\rangle$ in the first and third quadrants around the structure center, and smaller regions of negative $\left\langle u_{\mathrm{r}} v_{\mathrm{r}}\right\rangle$ in the second and fourth quadrants consistent with the conceptual schematic shown in figure $15(\mathrm{~b})$.

We need to consider transports of momentum that cause both incoherent and coherent Reynolds stresses. Contours of coherent Reynolds stress $\left\langle u_{\mathrm{p}} v_{\mathrm{p}}\right\rangle$ are shown in figure 15 (d) for phase II only. These contours show alternate regions of positive and negative values. During phases I to IV, peak values of $\left\langle u_{\mathrm{p}} v_{\mathrm{p}}\right\rangle$ are greater than the corresponding $\left\langle u_{\mathrm{r}} v_{\mathrm{r}}\right\rangle$ values in both planes. This indicates that, during the initial stages of the interaction, large-scale transport by coherent structures dominates the flow dynamics. Even when $\left\langle u_{\mathrm{p}} v_{\mathrm{p}}\right\rangle$ is larger, the time average may not be large because of the alternate signs in $\left\langle u_{\mathrm{p}} v_{\mathrm{p}}\right\rangle$. At phase $\mathrm{V}$, values of $\left\langle u_{\mathrm{r}} \boldsymbol{v}_{\mathrm{r}}\right\rangle$ and $<u_{\mathrm{p}} v_{\mathrm{p}}>$ are found to be comparable, indicating that with increasing distance coherent and incoherent motions become equally significant in the tansport of momentum. 


\subsubsection{Coherent Turbulence Production.}

Shear and normal production terms, $\left\langle\mathrm{P}_{s}>\left(\equiv-\left\langle u_{\mathrm{r}} v_{\mathrm{r}}\right\rangle(\partial<v>/ \partial x+\partial<u>/ \partial y)\right)\right.$ and $\left\langle\mathrm{P}_{\mathrm{n}}\right\rangle(\equiv-$ $\left\langle u_{\mathrm{r}}^{2}\right\rangle \partial\left\langle u>/ \partial x-\left\langle v_{\mathrm{r}}^{2}\right\rangle \partial\langle v\rangle / \partial y\right)$, are dependent on the coordinate system chosen while the total production $\langle\mathrm{P}\rangle\left(\equiv\left\langle\mathrm{P}_{\mathrm{s}}\right\rangle+\left\langle\mathrm{P}_{\mathrm{n}}\right\rangle\right)$ is invariant under rotation of the axes, and is therefore a more meaningful quantity. However, a detailed study of the distributions of turbulence production terms $\left\langle\mathrm{P}_{\mathrm{s}}\right\rangle$ and $\left\langle\mathrm{P}_{\mathrm{n}}\right\rangle=\left\langle\mathrm{P}_{\mathrm{nu}}\right\rangle+\left\langle\mathrm{P}_{\mathrm{nv}}\right\rangle$ (where $\left\langle\mathrm{P}_{\mathrm{nu}}\right\rangle=-\left\langle u_{\mathrm{r}}^{2}\right\rangle \partial\langle u\rangle / \partial x$ and $\left\langle\mathrm{P}_{\mathrm{nv}}\right\rangle=-\left\langle v_{\mathrm{r}}^{2}\right\rangle \partial\langle v\rangle / \partial y$ ) in an appropriate coordinate system is very helpful in understanding the physical mechanism of production in turbulent shear flows. Here we discuss these measures in the cartesian $(x, y)$ coordinates.

Contours of $\left\langle\mathrm{P}_{\mathrm{s}}\right\rangle,\left\langle\mathrm{P}_{\mathrm{n}}\right\rangle$ and $\langle\mathrm{P}\rangle$ are shown in figure 16(a-c). Since both incoherent Reynolds stress $\left\langle u_{\mathrm{r}} v_{\mathrm{r}}\right\rangle$ and coherent strain rate $\langle\mathrm{S}\rangle$ (not shown) have their maxima in the saddle regions, the peak of shear production $\left\langle\mathrm{P}_{s}\right\rangle$ necessarily occurs there. $\left\langle\mathrm{P}_{n}\right\rangle$ contours are quite different from $\left\langle\mathrm{P}_{\mathrm{s}}\right\rangle$ contours: peaks in $\left\langle\mathrm{P}_{\mathrm{n}}\right\rangle$ distributions occur not in the saddle region, but near the structure boundary. A comparison between production contours in the major and minor planes shows that peak values of $\left\langle\mathrm{P}_{5}\right\rangle$ are about 4 times higher in the major plane, while the peak values of $\left\langle\mathrm{P}_{\mathrm{n}}\right\rangle$ are about 2 times higher in the minor plane, resulting in comparable peak values of the total production $\langle\mathrm{P}\rangle$ in both planes. Furthermore, most of the negative production is produced by normal stresses.

A notable observation from these data is that normal and shear productions are comparable in their peak values, even though the net contribution of $\left\langle P_{n}\right\rangle$ to an area average is considerably lower because of cancellation between positive and negative valued regions. Coherent structure studies in the far field of jets (Tso 1983), wakes (Hayakawa \& Hussain 1985) and mixing layers (Metcalfe et al. 1987, Hayakawa \& Hussain 1990) have found $\left\langle\mathrm{P}_{n}\right\rangle$ to be very small and $\left\langle\mathrm{P}_{s}\right\rangle$ to contribute most heavily to $\langle\mathrm{P}>$. The apparent disagreement between those studies and the present one can be reconciled by examining the effects of curvature of vortical structures on the terms contributing to normal production. In cylindrical coordinates (figure 17a), shear and normal productions, in comparison with the case in rectangular coordinates, have two additional terms, e.g., $\left\langle u_{\mathrm{r}} \cdot v_{\mathrm{r}}{ }^{2}\right\rangle / 2 \mathrm{y}$ and $\left\langle v_{\mathrm{r}} \cdot v_{\mathrm{r}}^{2}\right\rangle / 2 \mathrm{y}$. The experiments were performed in a cartesian frame of reference; only later did we realize that a cylindrical coordinate system was more appropriate and that these two terms had not been measured. However, several arguments might be put forth to justify that the contributions of these two terms to $\langle\mathrm{P}\rangle$ _are negligible, and here we only consider the more significant terms $\left\langle\mathrm{P}_{\mathrm{s}}\right\rangle$, $<\mathrm{P}_{\mathrm{nu}}>$ and $<\mathrm{P}_{\mathrm{nv}}>$.

If the spatial distributions of $\left\langle u_{\mathrm{r}}{ }^{2}\right\rangle$ and $\left\langle v_{\mathrm{r}}{ }^{2}\right\rangle$ are very similar to each other, the normal production term can be written as

$$
\left\langle\mathrm{P}_{\mathrm{n}}\right\rangle=-\left\langle u_{\mathrm{r}}^{2}\right\rangle \partial\langle u\rangle / \partial x-\left\langle v_{\mathrm{r}}^{2}\right\rangle \partial\langle v\rangle / \partial y \cong-\left\langle u_{\mathrm{r}}^{2}\right\rangle(\partial\langle u\rangle / \partial x+\partial\langle v\rangle / \partial y+\langle v\rangle / y)+\left\langle u_{\mathrm{r}}^{2}\right\rangle\langle v\rangle / y .
$$

By continuity, the first term on the far right hand side vanishes, while the magnitude of the second term depends on the radius of curvature of the vortical structures. Thus, in a plane mixing layer and 
a plane wake, or in the far field of a circular jet, where the radius of curvature is large, contribution of $\left\langle\mathrm{P}_{\mathrm{n}}\right\rangle$ becomes negligible. However, in the near fields of elliptic and circular jets, the $\langle v\rangle / y$ contribution to $\left\langle\mathrm{P}_{\mathrm{n}}\right\rangle$ can be significant, resulting in positive $\left\langle\mathrm{P}_{\mathrm{n}}\right\rangle$ in the region of positive $\langle v>$ and negative $\left\langle\mathrm{P}_{n}\right\rangle$ in the region of negative $\langle v\rangle$. Of course, the distributions of $\left\langle u_{r}{ }^{2}\right\rangle$ and $\left\langle v_{r}{ }^{2}\right\rangle$ are not exactly the same, and therefore their differences also contribute to $\left\langle\mathrm{P}_{\mathrm{n}}\right\rangle$, but these differences are found to be small in situations where structures interaction is nominal, as in the studies cited above.

Mechanism of turbulence production. In the following, we examine the mechanism of turbulence production due to the interaction of the coherent strain field and incoherent turbulence. Let us consider the azimuthal cross-section of a vortical structure. In this plane, if one rotates the $(x, y)$ axes, the values of $\left\langle\mathrm{P}_{\mathrm{s}}\right\rangle,\left\langle\mathrm{P}_{\mathrm{nu}}\right\rangle$ and $\left\langle\mathrm{P}_{\mathrm{nv}}\right\rangle$ are redistributed among themselves such that the total production $\langle\mathrm{P}>$ remains invariant. By aligning axes at a braid (vortex sheet between vortical structures) such that the new coordinates $\left(x_{1}, y_{1}\right)$ are aligned with the diverging separatrix (where straining motion $\left.\partial<u_{1}>/ \partial y_{1}=\partial<v_{1}>/ \partial x_{1}=0\right)$, the total production $\langle\mathrm{P}\rangle$ can be made equal to $\left\langle\mathrm{P}_{\mathrm{n}}\right\rangle$ in the braid. This suggests that the physical mechanism of production is that of vortex stretching aligned in that direction. Guided by coherent production data, Hussain (1984) suggested that the saddle region is not a two-dimensional continuous sheet but consists of slender discrete vortices (ribs) aligned with the diverging separatrix. This conjecture found support in the low Re transitional pictures of Bernal \& Roshko (1986) and in direct simulation of turbulent flows (Hussain 1986). The interaction of ribs and rolls causes continual stretching of the ribs, resulting in the production of turbulence. In the usual streamwise, transverse and spanwise $(x, y, z)$ coordinate system, $\left\langle\mathrm{P}_{s}\right\rangle$ is the greatest contributor to the total production in the saddle region due to the stretching of the rib vorticity. The $\left\langle\mathrm{P}_{\mathrm{s}}\right\rangle$ contours confirm that most of the turbulence produced by incoherent Reynolds stress occurs near the saddles.

Effects of structure curvature on normal production. The mechanism of turbulence production by normal stresses and the effects of curvature of the roll are examined next. Let us first consider the cross-section of a two dimensional roller structure, whose distributions of $\left\langle\omega_{2}\right\rangle,\langle v\rangle$, $\partial<u>/ \partial x$ and $\partial<v>/ \partial y$ are qualitatively shown in figures $17(\mathrm{~b}-\mathrm{e})$. In these figures, vortex centers are marked with a ' + ', and positive and negative contours of $\partial\langle\|\rangle / \partial \mathrm{x}$ and $\partial\langle v\rangle / \partial \mathrm{y}$ are denoted by solid and dashed lines respectively. As ribs wrap around rolls, they undergo stretching or compression depending upon the sign of strain rates $\partial<u>/ \partial x$ and $\partial\langle v>/ \partial y$. The positive strain rate $\partial\langle u\rangle / \partial x$ causes stretching of the ribs in the streamwise direction, augmenting mostly $v$ and $w$ fluctuations associated with the ribs. [This is also apparent from the definition of $\left\langle\mathrm{P}_{n v}\right\rangle$, because $\left\langle\mathrm{P}_{n v}\right\rangle=-$ $\left\langle v_{\mathrm{r}}{ }^{2}\right\rangle \partial\langle v\rangle / \partial y=\left\langle v_{\mathrm{r}}{ }^{2}\right\rangle \partial\langle u\rangle / \partial x$.] Similarly, strain rate $\partial<v>/ \partial y$ augments $u$ and $w$ fluctuations by stretching the ribs in the transverse direction. In the case of two-dimensional roller structures, 
however, the total contribution to $\left\langle\mathrm{P}_{\mathrm{n}}>\right.$ is very small because stretching of a rib by $\partial\langle u\rangle / \partial x$ at a given location is associated with the same amount of compression by $\partial<v>/ \partial y$, and vice versa.

For a curved roller structure, tangential velocity must increase on the inner (high-speed) side and decrease on the outer (low-speed) side to accommodate the same flow between two radial planes crossing the vortex (see figure 17a). That is, the streamwise flow undergoes a larger acceleration and deceleration on the imner side than on the outer side. This is also evident from the equation of continuity in cylindrical coordinates. Because of the term $\langle v\rangle / y$, the strain rate $\partial\langle u\rangle / \partial x$ acquires higher peak values on the inner side of the structure than on the outer side. Contours of $\partial<u>/ \partial x$ for a curved roller structure are shown qualitatively in figure 17(f). Such a strain rate field produces higher stretching and compression of the ribs on the inner side of a curved vortical structure than those on the outer side. [ In figure 17(a), approximate rib-roll configuration is also superimposed for reference.] Preliminary studies of a circular jet using direct numerical simulation (Melander 1990) show clearly the effects of curvature on the distribution of $\partial<u>/ \partial x$. Distributions of $\partial<u>/ \partial x, \partial<v>/ \partial y$ and $<v>/ y$ obtained in this simulation are shown in figures $18(\mathrm{a}-\mathrm{c})$. In these figures, a few contours of $\left\langle\omega_{z}\right\rangle$ are superimposed for common reference. Contours of $\partial\langle v\rangle / \partial y$ show a clover-leaf distribution with alternating positive and negative regions with little difference in their peak magnitudes. Because $\langle v>/ y$ is nonzero, contours of $\partial\langle u\rangle / \partial x$ show a higher peak value on the inner side than on the outer side.

As a representative case, let us next examine the strain rate fields of interacting structures at phase II in the minor plane of the elliptic jet. This is deduced by superposing the strain field (in figure 17f) for two vortical structures. Qualitative contours of $-\partial\langle u\rangle / \partial x$ of individual structures and their resultant are shown in figures $19(\mathrm{a}, \mathrm{b})$. Qualitative contours of $\left\langle u_{\mathrm{r}}{ }^{2}\right\rangle$ (similar to figure 12) and $\left\langle\mathrm{P}_{\mathrm{nu}}\right\rangle=-\left\langle u_{\mathrm{r}}{ }^{2}\right\rangle \partial\langle u\rangle / \partial x$ are shown in figures $19(\mathrm{c}, \mathrm{d})$. Similar contours for $-\partial\langle v\rangle / \partial x$, $\left\langle v_{\mathrm{r}}{ }^{2}\right\rangle$ and $\left\langle\mathrm{P}_{\mathrm{nv}}\right\rangle$ are shown in figures $19(\mathrm{e}-\mathrm{h})$. Note that the deduced contours of $\left\langle\mathrm{P}_{\mathrm{nu}}\right\rangle$ and $\left.<\mathrm{P}_{\mathrm{nv}}\right\rangle$ show significant differences between them. Contours of $\left\langle\mathrm{P}_{\mathrm{nu}}\right\rangle$ (figure $19 \mathrm{~d}$ ) show one dominant positive region (marked A1) on the downstream side and two negative regions (marked N2 and N3) on the upstream side of the structures, while $\left\langle\mathrm{P}_{n v}\right\rangle$ contours (figure 19h) show clover-leaf distribution with alternating positive and negative regions around each structure center (marked $A 4$, $A 6, N 4, N 6$ and A5, A7, N5, N7). Considering such strain fields and the regions where the ribs joins the rolls, the regions of $\left\langle\mathrm{P}_{n u}\right\rangle$ and $\left\langle\mathrm{P}_{\mathrm{nv}}\right\rangle$ are shown schematically in figures $19(\mathrm{i}, \mathrm{j})$, respectively. In figure 19 (i), the ribs in the interacting regions labelled $A 1$ and $A 2$ are stretched in the $y$-direction resulting in positive $\left\langle\mathrm{P}_{\text {nu }}\right\rangle$, while compression of the ribs in regions $N 2$ and $N 3$ produces negative $\left\langle\mathrm{P}_{n u}\right\rangle$. In the interacting regions labelled $A 4$ and $A 7$ (figure 19j), the ribs are stretched in the $x$-direction, resulting in positive $\left\langle\mathrm{P}_{n v}\right\rangle$; compression of the ribs in regions N4, N5, N6 and $N 7$ produces negative $\left\langle\mathrm{P}_{n v}\right\rangle$. Furthermore, as the structure $r_{2}$ moves in the $x$-direction from phase $I$ to phase III, the section of the rib connecting the vortices $\Gamma_{1}$ and $\Gamma_{2}$ (denoted by $N$ ) is compressed. This region is thus characterized by negative values of $\left\langle\mathrm{P}_{n u}\right\rangle$ and $\left\langle\mathrm{P}_{n v}\right\rangle$. 
Experimentally obtained distributions of $\left\langle\mathrm{P}_{\mathrm{nu}}>\right.$ and $\left\langle\mathrm{P}_{\mathrm{nv}}>\right.$ (figures $19(\mathrm{k}, \mathrm{l})$ ) are fairly similar to the inferred distributions (figures $19(\mathrm{~d}, \mathrm{~h}, \mathrm{i}, \mathrm{j})$. For example, note the similarity of $\left\langle\mathrm{P}_{\mathrm{nu}}>\right.$ distributions in regions marked A1, A2, N2 and N3 in figures $19(\mathrm{~d}, \mathrm{i}, \mathrm{k})$, and distributions of $\left\langle\mathrm{P}_{\mathrm{nv}}\right\rangle$ in regions $A 4, A 7, N 4, N 5, N 6$ and $N 7$ in figures $19(h, j, 1)$. There are a few dissimilarities also; for example, regions $\mathrm{A} 3$ and $\mathrm{N} 1$ in figure $19(\mathrm{~d})$, and regions $\mathrm{A} 5$ in figure $19(\mathrm{~h})$ are not present in figures $19(\mathrm{k}, \mathrm{l})$. These differences between the inferred results using simple structure configuration and the experimentally obtained results are not unexpected; in reality structures are three-dimensional and their interactions produce more complex strain fields. Furthermore, three-dimensional motions resulting from the winding of ribs around rolls produce complex distributions of. $\left\langle\mathrm{P}_{n u}\right\rangle$ and $\left\langle\mathrm{P}_{n v}\right\rangle$.

Circular jet. For comparison with the elliptic jet, contours of $\left\langle\mathrm{P}_{\mathrm{s}}\right\rangle,\left\langle\mathrm{P}_{\mathrm{n}}\right\rangle$ and $\langle\mathrm{P}\rangle$ of the circular jet are shown in figure 20 for three phases (corresponding to the $\left\langle\omega_{Z}>\right.$ contours shown in figure $3 b$ ). These contour patterns are qualitatively similar to elliptic structures in the minor plane. However, there are some differences, especially in the later phases. For example, (a) unlike the elliptic jet, the circular jet shows regions of negative $\left\langle\mathrm{P}_{\mathrm{s}}\right\rangle$ in phase IV; (b) higher peak values of $\left\langle\mathrm{P}_{\mathrm{s}}\right\rangle$ in the first two phases of the circular jet; and (c) absence of positive $\left\langle\mathrm{P}_{n}\right\rangle$ near the bottom side of the trailing vortex of the circular jet (phase II), where the upstream rib joins with the roll. These differences are not unexpected because of the deformation of structures and a larger distance between the interacting structures in the minor plane of the the elliptic jet.

Peak levels of incoherent turbulence intensities in elliptic and circular jets are almost the same. However, a larger domain of the coherent structure cross-section, associated with incoherent turbulence intensities and net positive region of total production $\langle\mathrm{P}\rangle$, indicate that total turbulence production over the extent of the structure cross-section is greater in the elliptic jet than in the circular jet in all phases.

\subsection{Time-average characteristics}

Although time-average measures of various turbulence properties cannot address the detailed physics of turbulent flows, these measures are important in technological applications. These may be particularly important here because stable pairing enhances mixing. Furthermore, they serve as a data base for calibration of turbulence models and direct numerical simulations. Since time-average measures are the integrated footprint of advecting organized structures, one should expect that the spatial distributions of these measures can be explained, though not necessarily completely, in terms of coherent structures and their interactions. The motivation for this section is to investigate how the time-average measures are modified by stable pairing and to examine them in terms of coherent structures. 


\subsubsection{Longitudinal velocity.}

Contours of longitudinal mean velocity $U$, nondimensionalized by both local centerline mean velocity $U_{c}$ and exit velocity $U_{c}$, are shown in figures $21(a, b)$ respectively. The contours of $U / U_{c}$ illustrate that excitation at $\mathrm{St}_{\mathrm{De}}=0.85$ causes the outer boundary of the jet (e.g. the $\mathrm{U} / \mathrm{U}_{\mathrm{c}}=0.1$ line) to be pushed outward in both the major and minor planes with respect to the unexcited state. The inner boundary of the mixing layer (e.g. the $U / \mathrm{U}_{\mathrm{c}}=0.9$ line) is pushed outward everywhere in the minor plane, while in the major plane, it is pushed initially toward the centerline for $x / \mathrm{D}_{\mathrm{c}}<5$, but outward farther downstream. The shift in the inner boundary due to excitation is not as significant as the outer boundary. [Note that the contour $U / U_{c}=0.5$ represents the conventional jet half widdh.] These contours demonstrate enhanced spreading of the jet by the excitation. For example, the shear layer thickness at $x / \mathrm{D}_{\mathrm{e}}=5$ is increased by about $50 \%$ in the major plane and by about $20 \%$ in the minor plane. The inward and outward inclinations of $U / U_{c}$ contours in both planes are consistent with the vortex trajectories shown in figure 6(a). In the major plane, the motion of the vortices toward the jet axis produces inward inclinations of the higher-level $U / U_{c}$ contours, while outward motion of the leading vortex produces outward shifts of the $U / U_{c}$ lines in the minor plane. Excitation causes humps in the $\mathrm{U} / \mathrm{U}_{\mathrm{c}}$ contours at $x / \mathrm{D}_{\mathrm{e}} \cong 2.5$ in the minor plane where the trailing vortex rushes inside the leading one.

Since the centerline velocity $U_{c}$ decreases beyond the potential core of the jet, $U / U_{c}$ contours do not show the effect of excitation on the shortening of the potential core. This effect as well as the increase in the shear layer width by excitation is more clearly apparent in the $U / U_{e}$ contours (figure $21 b$ ). Note that the location where the $\mathrm{U} / \mathrm{U}_{\mathrm{e}}=0.95$ line intersects the jet axis has moved from $x / \mathrm{D}_{\mathrm{e}} \cong$ 5 to 2 due to the excitation; excitation considerably shortens the potential core.

The thickening of the shear layer by excitation is more pronounced in the major plane than in the minor plane. It was mentioned in section 4.1 that, although the vortex cores in the major plane advect toward the jet axis due to self-induction, these vortices leave behind low-vorticity fluid, which diffuses into ambient fluid before being entrained, causing the shear layer to grow further in the outer region. In the minor plane, the fact that the leading vortex moves almost monotonically away from the jet axis (see vortex trajectories; figure 6a) causes a continuous spreading of the low-level contour $\left(U / U_{e}=0.1\right)$. The higher-level $U / U_{e}$ contours are influenced mostly by the acceleration and deceleration of the trailing vortex, producing a hump (for example, see the 0.6 contour).

\subsubsection{Transverse velocity.}

For the unexcited jet, the $\mathrm{V} / \mathrm{U}_{\mathrm{c}}$ contours (figure 22 ) in the major plane show a large region of low negative values (denoted by dashed lines), while in the minor plane all of the contours are positive; the peak value of $\mathrm{V} / \mathrm{U}_{\mathrm{e}}$ is about 5 times higher in the minor plane. Excitation modifies $\mathrm{V} / \mathrm{U}_{\mathrm{c}}$ contours drastically in both planes. Note that $\mathrm{V}$ becomes positive everywhere in the major plane. 
The mean $V$ field is the result of the superposition of the self-induced motion of vortical structures and the rotational motion within the structures. In the unexcited state, pairing occurs intermittently and the pairing location is not fixed in space. Furthermore, the core vorticity is not as concentrated in the unexcited case as in the excited jet. As a result, one would expect a lower level of $V$ in the unexcited jet. In the major plane, since the vortices move toward the jet axis by selfinduction, the positive $v$ region in front of the structures is further diluted, while the negative $v$ region at the back becomes accentuated. Thus the dominance of the negative $v$ field associated with advecting structures results in only negative time average values of $\mathrm{V}$. Conversely, as the structures in the minor plane move away from the jet axis, they contribute to positive $V$ in this plane.

Under excitation, stronger ejection in front of interacting structures seems to dominate inward ingestion, producing only a positive $\mathrm{V}$ region in the major plane. This is apparent from the distributions of $\langle v\rangle$ (figure 9). The peak values and the area enclosed are higher for positive $\langle v\rangle$ contours than for negative $\langle v>$ contours. Inward motion of the core region, however, decreases the values of positive $\mathrm{V}$. This motion causes a valley in the $\mathrm{V}$ distribution (shown by hatched lines in figure 22), but is not strong enough to produce negative $V$. In the minor plane, excitation increases the peak value of $\mathrm{V}$ by a factor of about two. Closer to the jet axis, a small negative region of $V$ appears where the trailing vortex rushes through the leading vortex, producing stronger inward motion behind it.

\subsubsection{Turbulence intensities.}

Figure 23 shows that for the unexcited jet, the longitudinal turbulence intensity $\left(u^{\prime} / \mathrm{U}_{\mathrm{c}}\right)$ distributions have a single peak in each plane. This is because the formation and breakdown of structures are not localized in space. Since these events (formation and breakdown of structures) are spatially localized by the excitation, $u^{\prime} / \mathrm{U}_{c}$ contours show two peak regions in the shear layer; the first peak (closer to the jet exit) in both planes corresponds to structure roll-up. The second peak in the major plane corresponds to the breakdown of paired vortices. In the minor plane, the second peak is due to the breakdown of leading vortices. Note that the contours_have a valley near the jet axis. Downstream of the valley, the contour levels increase and then decrease. Along the jet axis, the first peak in $u^{\prime} / U_{c}$ is primarily induced by the accelerating trailing vortices as they rush through the leading ones. The valley corresponds to the deceleration phase of the trailing vortices and the peak region downstream of the valley is due to breakdown of the trailing vortices.

Like $u^{\prime} / \mathrm{U}_{\mathrm{e}}$, the $v^{\prime} / \mathrm{U}_{\mathrm{e}}$ contours in the unexcited jet (figure 24) show a single peak in each plane for the same reason mentioned above. Under excitation, $v^{\prime} /$ Ue contours also show two peaks in the shear layers of both planes, and a valley (shown hatched) in the minor plane. However, the contour details of $v^{\prime} / \mathrm{U}_{\mathrm{e}}$ are quite different from those of $u^{\prime} / \mathrm{U}_{\mathrm{c}}$, especially near the jet axis. The valley in the $v^{\prime}$ distributions is transversely away from the jet axis, and downstream of the valley there is another peak (i.e. a third peak) in the minor plane. The first peak in $u^{\prime}$ along the jet axis $\left(x / D_{e} \cong 2.5\right)$ is 
mostly due to the induced motion $u_{\mathrm{p}}=(\langle u\rangle-\mathrm{U})$ because large $u_{\mathrm{p}}$ variations occur on the jet axis also. On the other hand, the positive and negative peak values of $v_{\mathrm{p}}=(\langle v\rangle-\mathrm{V})$ occur upstream and downstream of advecting structures, and away from the jet axis (see figure 9; distributions of $v_{\mathrm{p}}$ and $\langle v\rangle$ are similar because of very low values of $\mathrm{V}$ ), causing the peak and valley of $v^{\prime} / \mathrm{U}_{\mathrm{e}}$ to occur away from the jet axis. Because the $v_{\mathrm{p}}$ distribution is symmetric about the jet axis and is zero along the jet axis, the $v^{\prime}$ distribution shows a lower peak value than does the $u^{\prime}$ distribution along the jet axis. This is consistent with data in jets of other geometries, e.g. circular (Wygnanski \& Fiedler 1969), planar (Gutmark \& Wygnanski 1976) and rectangular (Krothapalli et al. 1981).

\subsubsection{Reynolds stress.}

Figure 25 shows that excitation produces regions of negative Reynolds stress in both planes. The appearance of the negative $\bar{u} \bar{v}$ close to the exit plane is quite evident from the phase-average measures of $\left\langle u_{\mathrm{r}} v_{\mathrm{r}}\right\rangle$ and $\left\langle u_{\mathrm{p}} v_{\mathrm{p}}\right\rangle$ (figures 14 and 15e), since the time-average Reynolds stress is equal to the average of coherent Reynolds stress $\langle u v\rangle\left(\equiv\left\langle u_{\mathrm{r}} v_{\mathrm{r}}\right\rangle+\left\langle u_{\mathrm{p}} v_{\mathrm{p}}\right\rangle\right)$ over all phases. Excitation increases the positive peak of $\overline{u v}$ by about $60 \%$ in both of the planes. A comparison of $\left\langle u_{\mathrm{r}} v_{\mathrm{r}}\right\rangle$ and $\left\langle u_{\mathrm{p}} v_{\mathrm{p}}\right\rangle$ contours shows that most of the negative correlation is associated with $\left\langle u_{\mathrm{p}} v_{\mathrm{p}}\right\rangle$ (i.e. due to the large-scale motion of structures) during initial phases of pairing. Positive correlation occurs at the structure front where the jet fluid is ejected away from the jet axis while negative correlation occurs at the back of the structures where the ambient fluid is drawn inward. At phases when the trailing vortex moves inside the leading vortex, the region of negative correlation is more dominant than the region of positive correlation. Thus, under excitation, when pairing events are stabilized to occur at a particular spatial location, the time-average Reynolds stress also shows a region of negative correlation. Farther downstream, say $x / D_{e} \geq 4$, as the correlation of coherent motion (i.e. $\left\langle u_{\mathrm{p}} v_{\mathrm{p}}\right\rangle$ ) decreases, the weak negative regions of $\left\langle u_{\mathrm{p}} v_{\mathrm{p}}\right\rangle$ are overshadowed by the positive regions of $\left\langle u_{\mathrm{p}} v_{\mathrm{p}}\right\rangle$ and $\left\langle u_{\mathrm{r}} v_{\mathrm{r}}\right\rangle$, giving rise to only positive time-average $\overline{u v}$ contours. In unexcited jets, when the interaction phases which give rise to negative correlation occur randomly in space and time, contours of $\overline{u v}$ do not show negative regions because of dominance of positive regions of $\left\langle u_{\mathrm{p}} v_{\mathrm{p}}\right\rangle$ and $\left\langle u_{\mathrm{r}} v_{\mathrm{r}}\right\rangle$.

\subsubsection{Turbulence production.}

Contours of time-average shear production $\mathrm{P}_{s}$, normal production $\mathrm{P}_{n}$ and total production $\mathrm{P}$ are shown in figure $26(\mathrm{a}-\mathrm{c})$. In the unexcited case, since the strain rates $\partial U / \partial y$ and $\partial U / \partial x$ are much stronger than $\partial V / \partial x$ and $\partial V / \partial y$, mean turbulence production due to shear and normal stresses is predominantly contributed by the gradients of $\mathrm{U}$. The shear production is dominated by $\partial \mathrm{U} / \partial y$, which is negative in both planes, resulting in regions of only positive shear production. Note that most of negative production is generated by normal stresses. 
Excitation shows significant effects on the production contours. Regions of negative $P_{S}$ develop in both planes. As to be expected, the negative $P_{S}$ is much more dominant in the minor plane because of large counter-gradient transport of momentum during the phase when the trailing vortex passes through the leading one. In the unexcited case, the contribution of shear production is much higher than normal production (almost an order of magnitude), but excitation significantly increases the contribution of normal production.

Because of the dominance of positive values of $P_{s}$ and $P_{n}$ in the major plane of both excited and unexcited cases, the total production $P$ in this plane is only positive. In the excited case, the distributions of $\mathrm{P}$ in both planes are also evident from the phase average $\langle\mathrm{P}\rangle$ contours. Large regions of positive $\angle \mathrm{P}>$ in the major plane result in only positive regions of $\mathrm{P}$, while the dominance of both positive and negative regions of $\langle P\rangle$ is reflected on the time-average $P$.

\section{Concluding remarks}

Three-dimensional deformation, intrinsic to elliptic vortices because of the curvature-dependent self-induction, makes pairing in an elliptic jet more complicated than in a circular jet. Unlike circular jets, where pairing is nearly uniform around the entire perimeter of the vortices, pairing in elliptic jets occurs only in the major-axis sides. A large separation of interacting vortices in the minor plane hinders pairing; the trailing vortex, instead of pairing, rushes through the leading vortex and subsequently breaks down.

Since pairing takes place along a short segment in the major-axis side only, morphologically speaking, pairing does not occur through a leapfrog motion as in circular jets; rather it is better described as an entanglement process. In post-transition and fully-developed turbulent states of various shear flows, where the vortical structures are predominanlly three-dimensional, merger through entanglement is likely to be a common mode of vortex interaction. Other important modes of interactions are merger of opposite-signed vortices and the cut-and-connect interaction (which we have discussed elsewhere). By controlling the deformation and interaction of three-dimensional vortical structures in the elliptic jet, we could investigate this important mode of interaction in detail. It is quite reasonable to infer from the topology of vortex pairing that compared to leapfrogging processes, an entanglement process causes greater stretching of interacting vortices, producing greater enstrophy production and vorticity diffusion. Three-dimensional deformation of vortical structures and the resulting pairing through entanglement in the major plane and violent breakdown in the minor plane should cause better mixing in elliptic jets than in circular jets. This is evident from the coherent vorticity contours which show that vorticity is diffused over a larger area (almost twice) in elliptic jets than in circular jets. 
In the present study, although we have not educed rib substructures, the dynamics and importance of ribs in turbulence production mechanism have been inferred from the detailed measurements of turbulence production due to incoherent normal and Reynolds stresses. Prior studies of coherent structures in plane wakes and mixing layers, and the far field of circular jets have revealed that coherent production due to incoherent normal stresses is negligible compared to production due to incoherent Reynolds stress. However, in the near fields of elliptic and circular jets, where structure curvature is strong, curvature plays important roles in the production of turbulence by normal stresses.

The authors are grateful to Dr. James Bridges for careful reviews of the manuscript, and to Dr. Mogens Melander for providing results of numerical simulations. This work was supported by NASA-Lewis Research Center Grant No. NAG 3-639 and Department of Energy Grant No. DEFG05-88ER13839. 


\section{References}

Acton, E. 1976 J. Fluid Mech. 76, 561.

Ashurst, W.T. 1979 Turbulent Shear Flows I (ed. F. Durst et al.) Springer, p. 40.

Batchelor, G. K. \& Gill, A. E. 1962 J. Fluid Mech. 14, 529.

Bechert, D. \& Pfizenmaier, E. 1975 J. Sound and Vibration 43, 581.

Bernal, L. P. \& Roshko, A. 1986 J. Fluid Mech. 170, 499.

Bridges, J.E. 1988 (private communication).

Bridges, J.E. \& Hussain, A.K.M.F. 1987 J. Sound \& Vib. 117, 289.

Browand, F.K. 1966 J. Fluid Mech. 26, 281.

Browand, F.K. \& Weidman, P.D. 1976 J. Fluid Mech. 76, 127.

Brown, G.L. \& Roshko, A. 1974 J. Fluid Mech. 64, 775.

Bruun, H.H. 1977 J. Fluid Mech. 83, 641.

Cantwell, B. \& Coles, D. 1983 J. Fluid Mech. 136, 321.

Chandrasuda, C., Mehta, R. D., Weir, R. A. D. \& Bradshaw, P. 1978 J. Fluid Mech. 85, 693.

Clark, J. A. \& Kit, L. 1980 J. Fluid Engr. 102, 219.

Cohen, J. \& Wygnanski, I. 1987 J. Fluid Mech. 176221.

Corcos, G.M. \& Sherman, F.S. 1984 J. Fluid Mech. 139, 29.

Crighton, D. G. 1981 J. Fluid Mech. 106, 261.

Ffowcs-Williams, J.E. \& Kempton, A.J. 1978 J. Fluid Mech. 84, 673.

Grinstein, F. F., Hussuin, F. \& Oran, E.1989 AIA A paper No. 89-0977.

Gutmark, E. \& Wygnanski, 1. 1976 J. Fluid Mech. 38, 577.

Hayakawa, M. \& Hussain, A.K.M.F. 1985 Turbulent Shear Flows vol. 5, p.4.33. Cornell U.

Hayakawa, M. \& Hussain,.F. 1990 (submitted to J. Fluid Mech.).

Ho, C. M. \& Huang, L. S. 1982 119, 443.

Ho, C.M. \& Huerre, P. 1984 Ann. Rev. Fluid. Mech. 16, 365.

Husain, H.S. 1984 Ph. D. Thesis, University of Houston.

Hussain, A.K.M.F. 1981 Proc. Ind. Acad. Sci. 4, 129.

Hussain, A.K.M.F. 1983 Phys. Fluids 26, 2816.

Hussain, A.K.M.F. 1984 Turbulence and Chaotic Phenomena in Fluids (ed. T. Tatsumi) p. 453,

North Holland.

Hussain, A.K.M.F. 1986 J. Fluid Mech. 173, 303.

Hussain, A.K.M.F. \& Zaman, K.B.M.Q. 1980 J. Fluid Mech. 101, 493.

Hussain, A.K.M.F. \& Husain, H.S. 1989 J. Fluid Mech. 208, 257.

Jimenez, J., Cogollos, M. \& Bernal, L. 1985 J. Fluid Mech. 152, 125.

Kambe, T. 1984 J. Sound \& Vib. 95, 351.

Kelly, R. E. 1967 J. Fluid Mech. 27, 667.

Kibens, V. 1980 AIAA J. 18, 434. 
Krothapalli, A., Baganoff, D. \& Karamcheti, K. 1981 J. Fluid Mech. 107, 201.

Laufer, J. 1983 J. Appl. Mech. 50, 1079.

Laufer, J. 1974 Omaggio Carlo Ferrari, p.451.

Laufer, J. \& Zhang, J. Z. 1983 Phys. Fluids 26, 1740.

Mankbadi, R. R. 1985 J. Fluid Mech. 160, 385.

Mansour, N. N. \& Barr, P.K. 1985 5th Symposium on Turbulent Shear Flows, Ithaca NY.

Mansour, N.N., Hussain, F. \& Buell, J.C., 1988 CTR Report CTR-S88, p. 57.

Maxworthy, T. 1974 J. Fluid Mech. 64, 227.

Melander, M.V. 1990 (private communication)

Metcalfe, R., Hussain, A.K.M.F., Menon, S. \& Hayakawa, M. 1987 Fifth Symp. Turbulent

Shear Flows, vol. 5 (ed. F. Durst et al.) p. 110, Springer.

Möhring, W. 1978 J. Fluid Mech.85, 685.

Monkewitz, P. A. 1988 J. Fluid Mech. 188, 223.

Moore, C.J. 1977 J. Fluid Mech. 80, 321.

Obermeier, F. 1985 J. Sound \& Vib. 99, 111.

Patnaik, P.C. Sherman, F.S. \& Corcos, G.M. 1976 J. Fluid Mech. 73, 215.

Petersen, R. A. \& Samet, M. M. 1988 J. Fluid Mech. 194, 153.

Raman, G. \& Rice E. J. 1989 NASA Tech. Memorandum 101946.

Reynolds, W.C. \& Bouchard, E.E. 1981 Unsteady Turbulent Shear Flows (eds. R. Michel, J.

Cousteix \& R. Houdeville) p. 402, Springer.

Riley, J.J. \& Metcalfe, R.W. 1980 AIAA paper no. 80-0274.

Sato, H. 1959 J. Phys. Soc. Japan 14, 1797.

Tso, J. 1983 Ph.D. Thesis, Johns Hopkins University.

Wille, R 1963 Z. Flugwiss 11, 222.

Winant, C.D. \& Browand, F.K. 1974 J. Fluid Mech. 63, 237.

Wygnanski, I. \& Fiedler, F. 1969 J. Fluid Mech. 38, 577.

Yule, A.J. 1978 J. Fluid Mech. 89, 413.

Zaman, K.B.M Q. \& Hussain, A.K M.F. 1980 J. Fluid Mech. 101, 449.

Zaman,K.B.M.Q. \& Hussain, A.K.M.F. 1981 J. Fluid Mech. 112, 379.

Zaman,K.B.M.Q. \& Hussain, A.K.M.F. 1984 J. Fluid Mech. 138, 325. 


\section{Figure captions}

Figure 1 (a) Schematic of the flow facility and measurement scheme.

(b) Co-ordinate system.

Figure 2 (a) Evolution of $u$-spectrum along the jet centerline under excitation at $S t_{D e}=0.85$.

(b) Traces of $u(t)$ signal along the jet centerline under excitation at $S_{\mathrm{De}}=0.85$.

Figure 3 Contours of coherent azimuthal vorticity $\left\langle\omega_{z}>/ f\right.$ at various phases during pairing: (a) elliptic jet; (b) circular jet.

Figure 4 Distributions of vorticity peaks during pairing in elliptic and circular jets: (a) major plane; (b) minor plane; $\square$, leading vortex, elliptic jet; 0 , trailing vortex, elliptic jet ; ....., circular jet.

Figure 5 Areas enclosed by contours of various levels of vorticity $\left\langle\omega_{2}\right\rangle / f$ : (a) major plane;

(b) minor plane; (c) circular jet. 0 , phase I; $\square$, phase II; $\nabla$, phase III;

$\Delta$, phase IV; $O$, phase $V$.

(d) Area enclosed by the vorticity level $\left\langle\omega_{2}\right\rangle / f=1$. 口, major plane; $\mathbf{m}$, minor plane; o, circular jet; • circular jet (Hussain \& Zaman 1980).

Figure 6 (a) Trajectories of structures during pairing in elliptic and circular jets: $\circ$, leading vortex (minor plane); $\bullet$, trailing vortex (minor plane); $\nabla$, leading vortex (major plane); $\Delta$, trailing vortex (major plane); ....., circular jet.

Schematics showing motions of elliptic structures due to self- and mutual inductions:

(b) minor plane; (c) major plane

Figure 7 (a) Sequence of flow visualization pictures in the major and minor planes during pairing, presented in the form of isointensity contours.(b) Schematics of the vortex evolution process during pairing.

(c) Flow visualization pictures taken simultaneously in the major and minor planes during pairing.

Schemalics of the entanglement of vortices in: (d) plane jet; (e) circular jet.

Figure 8 Contours of phase-average longitudinal velocity $<u>/ U_{\mathrm{e}}$.

Figure 9 Contours of phase-average transverse velocity $\langle v\rangle / \mathrm{U}_{\mathrm{e}}$

Figure 10 Convection velocities of vortices during pairing. 0 , leading vortex (minor plane);

-, trailing vortex, (minor plane); $\nabla$, leading vortex (major plane); $\Delta$, trailing vortex (major plane); - - , trailing vortex (circular jet); _- - leading vortex (circular jet).

Figure 11 Distributions of phase-average velocity vectors during pairing.

Figure 12 Contours of incoherent longitudinal turbulence intensity $\left\langle u_{\mathrm{r}}^{2}>1 / 2 / \mathrm{U}_{\mathrm{e}}\right.$.

Figure 13 Contours of incoherent transverse turbulence intensity $<v_{\mathrm{r}}^{2}>1 / 2 / \mathrm{U}_{\mathrm{c}}$.

Figure 14 Contours of incoherent Reynolds stress $\left\langle u_{\mathrm{r}} v_{\mathrm{r}}\right\rangle / \mathrm{U}_{\mathrm{e}}{ }^{2}$. 
Figure 15 (a) Schematics showing the mechanism of positive and negative $\left\langle u_{\mathrm{r}} v_{\mathrm{r}}\right\rangle$ generation. (b, c) Schematics showing the regions of positive and negative $\left\langle u_{\mathrm{r}} v_{\mathrm{r}}\right\rangle$

(d) Contours of coherent Reynolds stress $\left\langle u_{\mathrm{p}} v_{\mathrm{p}}>/ \mathrm{U}_{\mathrm{e}}{ }^{2}\right.$

Figure 16 (a) Contours of coherent shear production $\left\langle\mathrm{P}_{\mathrm{s}}\right\rangle /\left(\mathrm{U}_{\mathrm{e}}^{2}\right.$.f).

(b) Contours of coherent normal production $\left\langle\mathrm{P}_{n}>/ \mathrm{U}_{\mathrm{e}}^{2} \cdot \mathrm{f}\right)$

(c) Contours of total coherent production $\left\langle\mathrm{P}>/ \mathrm{U}_{\mathrm{c}}^{2}\right.$.f

Figure 17 (a) Schematic of a ring vortex and cylindrical coordinates. Qualitative contours of: (b) $\left\langle\omega_{z}\right\rangle$; (c) $\langle v>$; (d) $\partial\langle u\rangle / \partial x$; (e) $\partial\langle v>/ \partial y$ for a two dimensional roller structure.

(f) Qualitative contours of $\partial<u>/ \partial$, showing the effects of structure curvature.

Figure 18 Numerical results of a circular jet.showing contours of: (a) $\partial<u>/ \partial x$; (b) $\partial<v>/ \partial y$; (c) $\langle v>/ y$. Solid and dashed lines are contours of positive and negative values respectively. ___ vorticity contours.

Figure 19 (a) Qualitative contours of interacting vortices at phase II: (a) superposition of $-\partial\langle u>\partial x$ of vortex 1 and vortex 2 ; (b) $-\partial<u>/ \partial x$; (c) $\left\langle u_{\mathrm{r}}^{2}\right\rangle$; (d) $\left\langle\mathrm{P}_{\mathrm{nu}}>\right.$; (e) superposition of $-\partial<v>/ \partial y$ of vortex 1 and vortex $2 ;(\mathrm{f})-\partial<v>/ \partial y ;(\mathrm{g})<v_{\mathrm{r}}^{2}>$; (h) $\left\langle\mathrm{P}_{\mathrm{nv}}>\right.$. Schematics showing how rib-roll interaction produce regions of: (i) $\left\langle\mathrm{P}_{n u}\right\rangle$; (j) $\left\langle\mathrm{P}_{n v}\right\rangle$. (k) Contours of the normal production term $\left\langle\mathrm{P}_{n u}>/\left(U_{e}^{2} . f\right)\right.$; phase II, minor plane. (l) Contours of the normal production term $\left\langle\mathrm{P}_{n v}\right\rangle /\left(\mathrm{U}_{\mathrm{c}}^{2}\right.$.f); phase II, minor plane.

Figure 20 Contours of $\left.\left\langle\mathrm{P}_{\mathrm{s}}>/ \mathrm{U}_{\mathrm{e}}^{2} . \mathrm{f}\right),\left\langle\mathrm{P}_{\mathrm{n}}\right\rangle / \mathrm{U}_{\mathrm{e}}^{2} . \mathrm{f}\right)$ and $\left\langle\mathrm{P}>/ \mathrm{U}_{\mathrm{e}}^{2} . \mathrm{f}\right)$ during pairing in the circular jet.

Figure 21 (a) Contours of $U / \mathrm{U}_{\mathrm{c}}$ of the excited $\left(\mathrm{St}_{\mathrm{Dc}}=0.85\right)$ and unexcited elliptic jets. - excited; -.. - unexcited.

(b) Contours of $\mathrm{U} / \mathrm{U}_{\mathrm{c}}$ of the excited $\left(\mathrm{St}_{\mathrm{Dc}}=0.85\right)$ and unexcited elliptic jets.

Figure 22 Contours of $\mathrm{V} / \mathrm{U}_{\mathrm{e}}$ of the excited $\left(\mathrm{St}_{\mathrm{Dc}}=0.85\right)$ and unexcited elliptic jets.

Figure 23 Contours of $u^{\prime} / \mathrm{U}_{\mathrm{c}}$ of the excited $\left(\mathrm{St}_{\mathrm{Dc}}=0.85\right)$ and unexcited elliptic jets.

Figure 24 Contours of $v^{\prime} / \mathrm{U}_{\mathrm{c}}$ of the excited $\left(\mathrm{St}_{\mathrm{Dc}}=0.85\right)$ and unexcited elliptic jets.

Figure 25 Contours of $\overline{u v} / \mathrm{U}_{\mathrm{c}}^{2}$ of the excited $\left(\mathrm{St}_{\mathrm{Dc}}=0.85\right)$ and unexcited elliptic jets.

Figure 26 Contours of time-average turbulence production in excited $\left(\mathrm{St}_{\mathrm{DC}}=0.85\right)$ and unexcited elliptic jets: (a) shear production; (b) normal production; (c) total production. 
(a) $\quad i:$

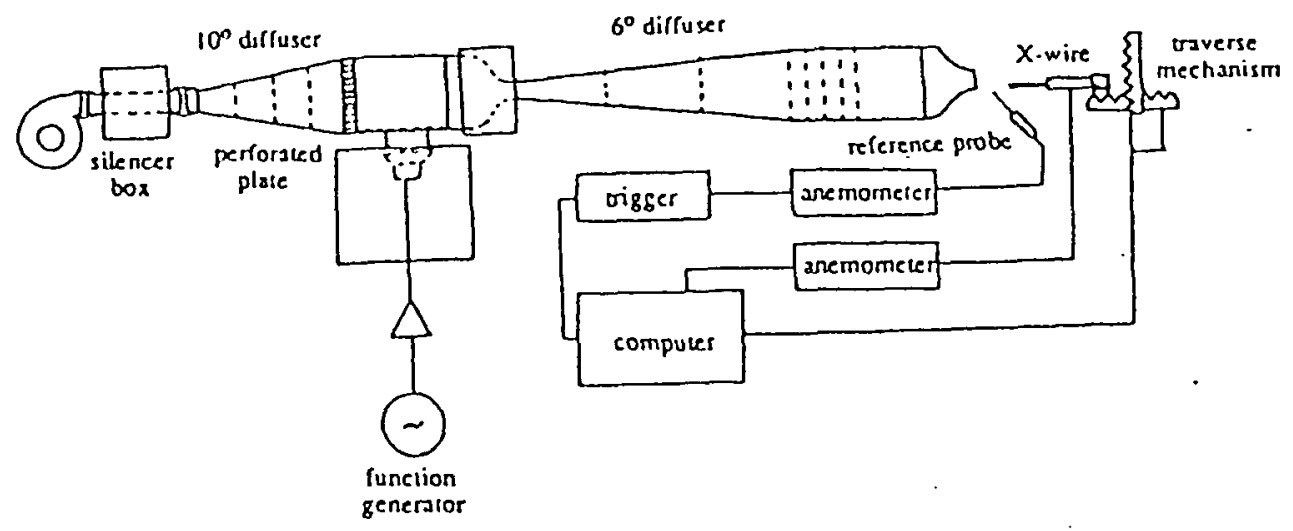

(b)

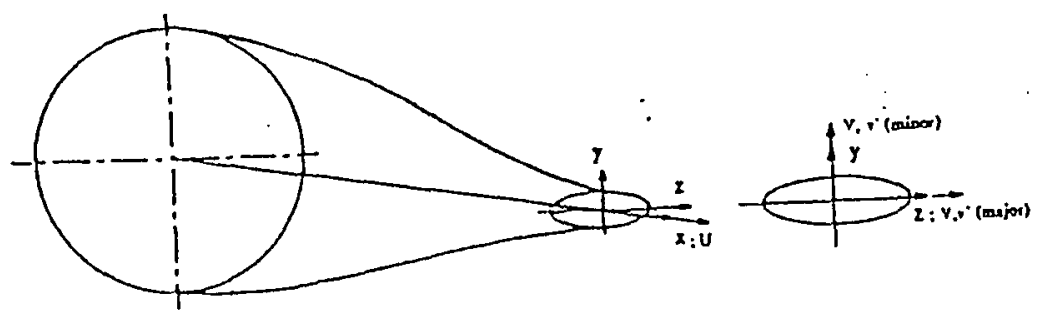

$\begin{array}{ll}\text { Figure } 1 & \text { (a) Schematic of the flow facility and measurement scheme. }\end{array}$

(b)

(a)

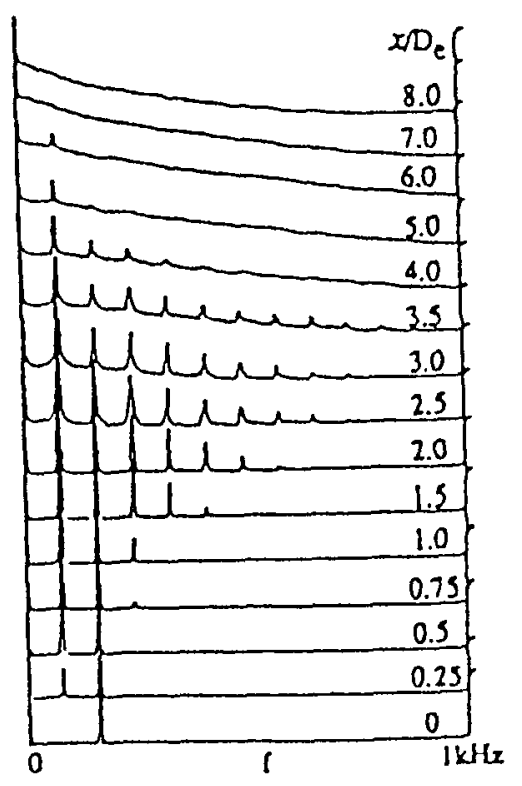

$x D_{e}$

0 mannmanimann

0.25 ב

0.5 nmonnmonmmoms

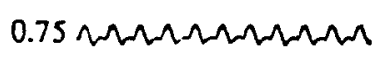

$1.0 \mathrm{MMMM}$ 1.25 WMMMMN

${ }_{1.5}$ WMMMM

${ }^{1.75}$ WMNMNA

2. MUMulum

${ }_{225}$ MUCuCuM $x / D_{c}$

N.5NANANAN

2.75NNWNNN

3. Mbroburous

3.2 Shrowhthk

3.5 Whitrown

${ }_{3.5}$ Nhdimadow

Figure 2 (a) Evolution of $u$-spectrum along the jet centerline under excitation at $S t_{D e}=0.85$.

(b) Traces of $u(t)$ signal along the jet centerline under excitation at $S t_{D e}=0.85$. 

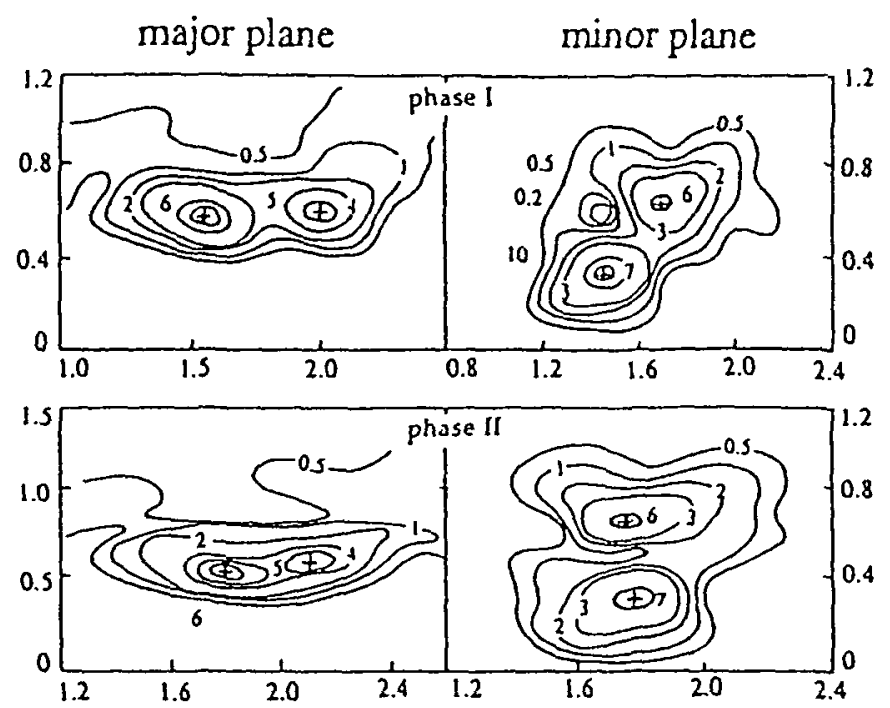

(a)
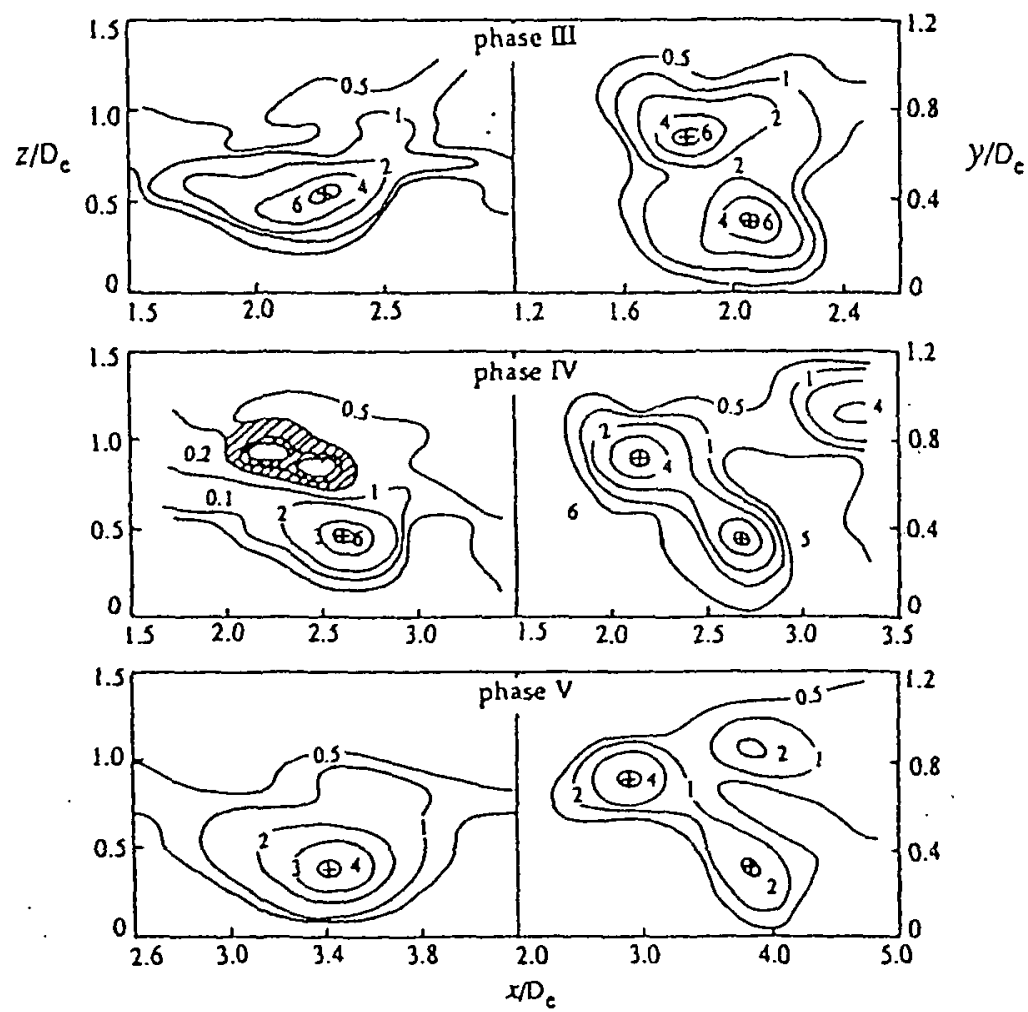

(b)
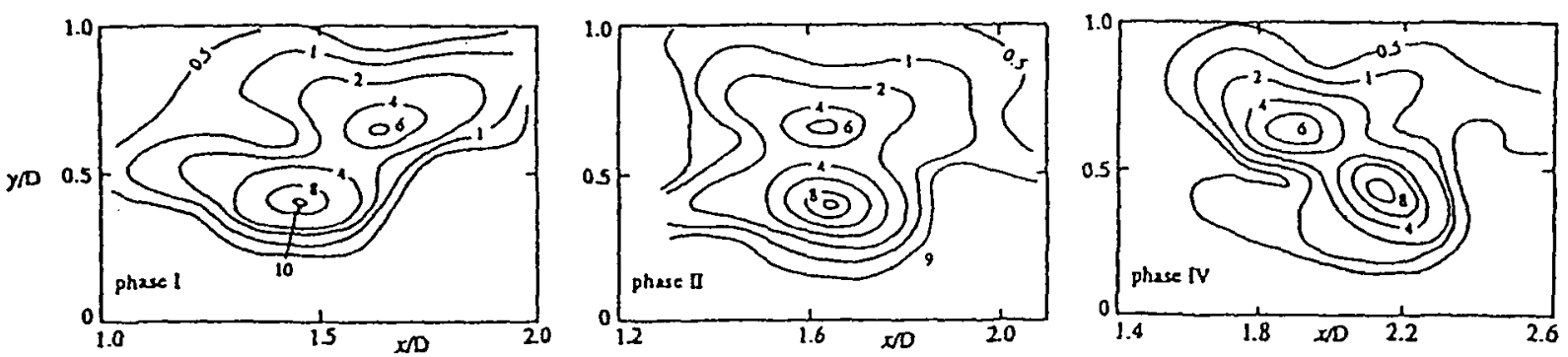

Figure 3 Contours of coherent azimuthal vorticity $<\omega_{z}>/ f$ at various phases during pairing: (a) elliptic jet; (b) circular jet. 


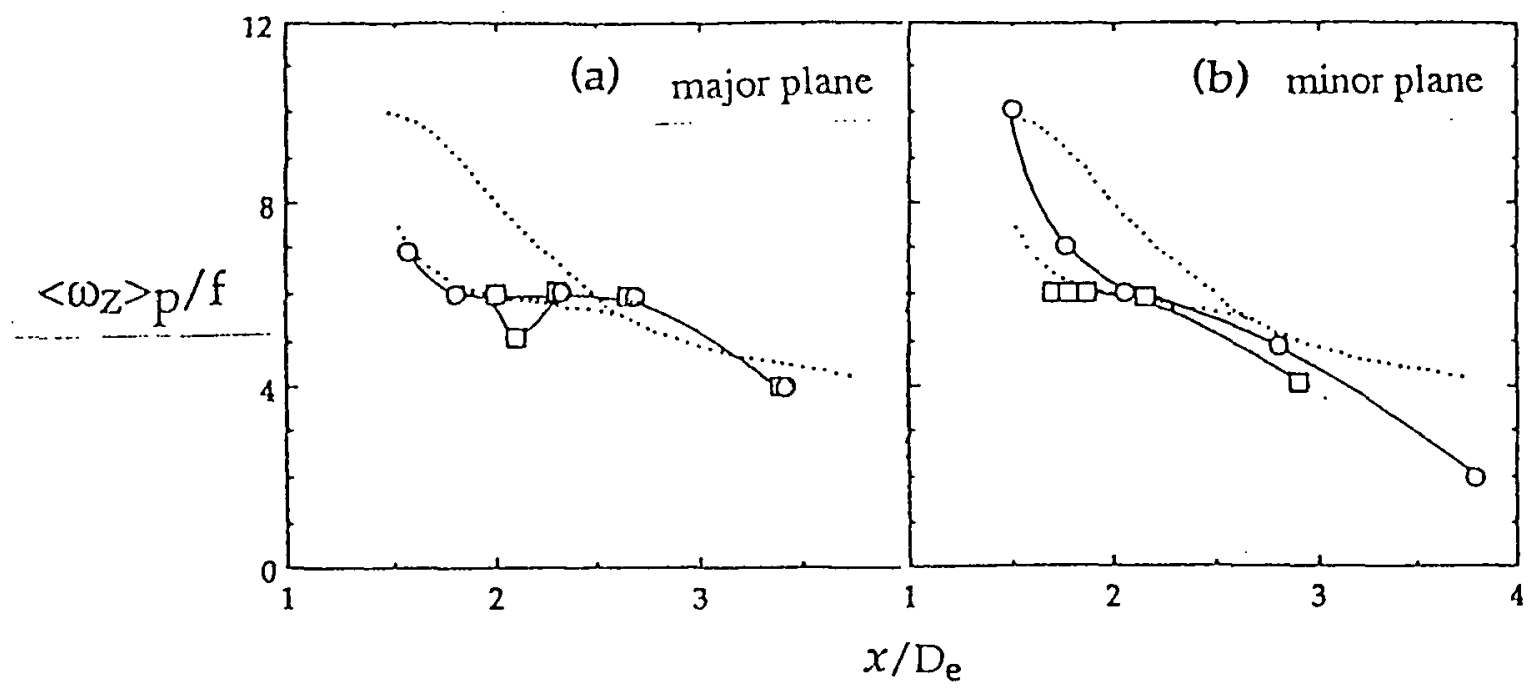

Figure 4 Distributions of vorticity peaks during pairing in elliptic and circular jets: (a) major plane; (b) minor plane; $\square$, leading vortex, elliptic jet; $O$, trailing vortex, elliptic jet ; ....., circular jet.
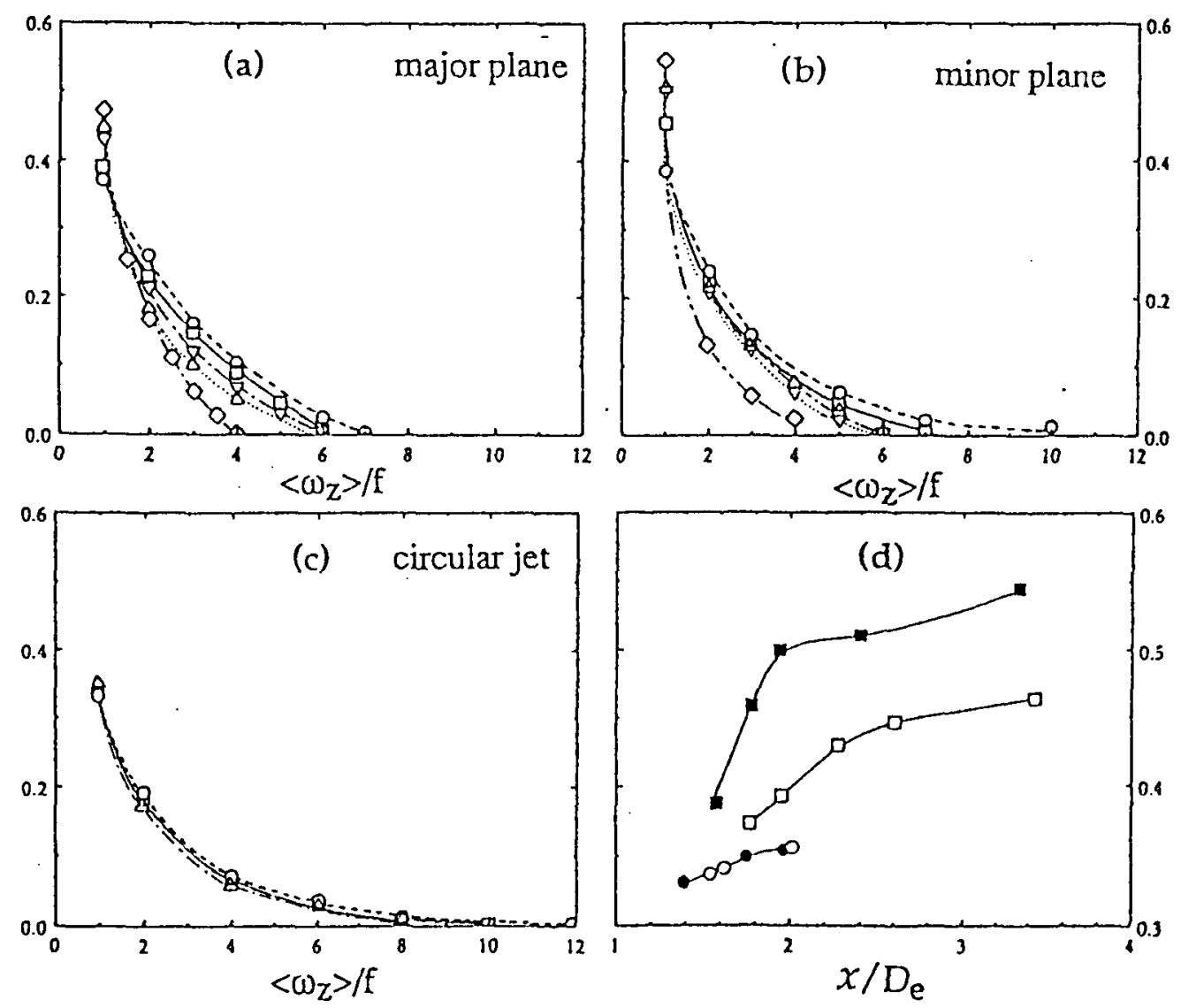

Figure 5 Areas enclosed by contours of various levels of vorticity $\left\langle\omega_{z}\right\rangle / f$ : (a) major plane; (b) minor plane; (c) circular jet. 0 , phase I; $\square$, phase $\Pi$; $\nabla$, phase III; $\Delta$, phase IV; $\diamond$, phase V.

(d) Area enclosed by the vorticity level $\left\langle\omega_{z}\right\rangle / f=1$. $\square$, major plane; $\mathbf{m}$, ninor plane; $\circ$, circular jet; • circular jet (Hussain \& Zaman 1980). 


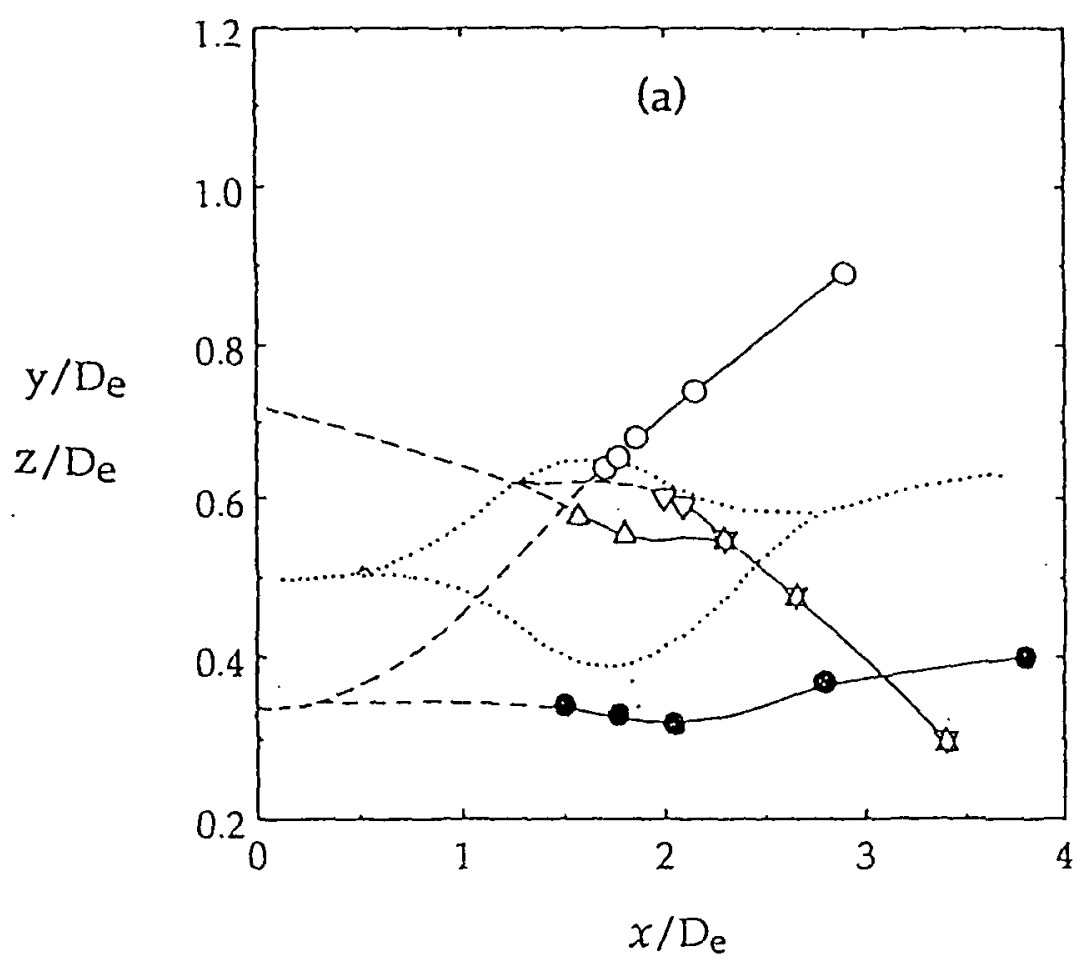

(b)
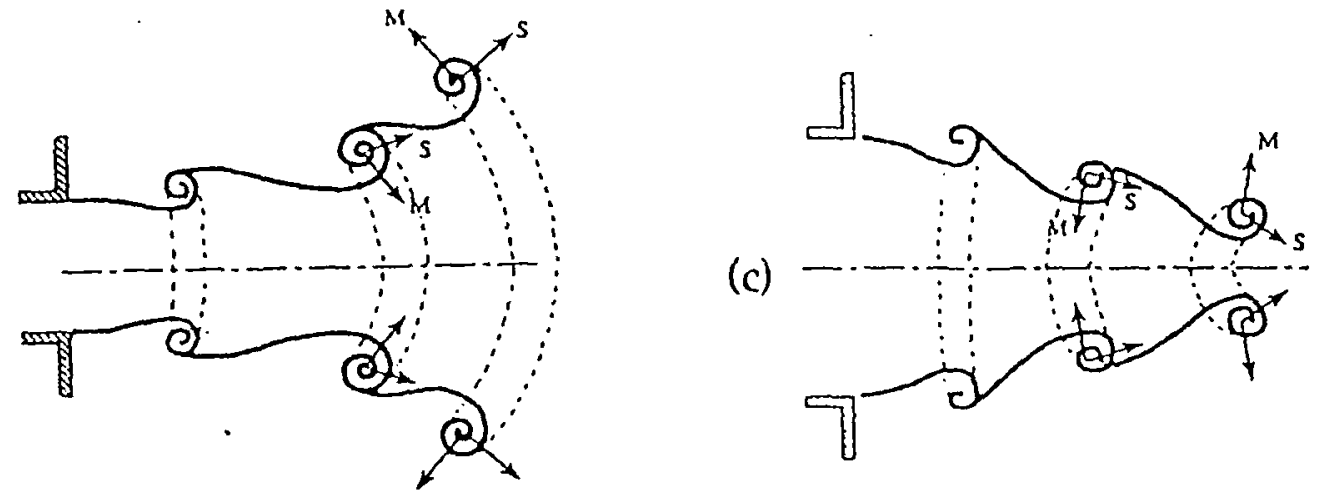

Figure 6 (a) Trajectories of structures during pairing in elliptic and circular jets:

$\circ$, leading vortex (minor plane); $\bullet$, trailing vortex (minor plane); $\nabla$, leading vortex (major plane); $\Delta$, trailing vortex (major plane); ....., circular jet.

Schematics showing motions of elliptic structures due to self- and mutual inductions:

(b) minor plane; (c) major plane 


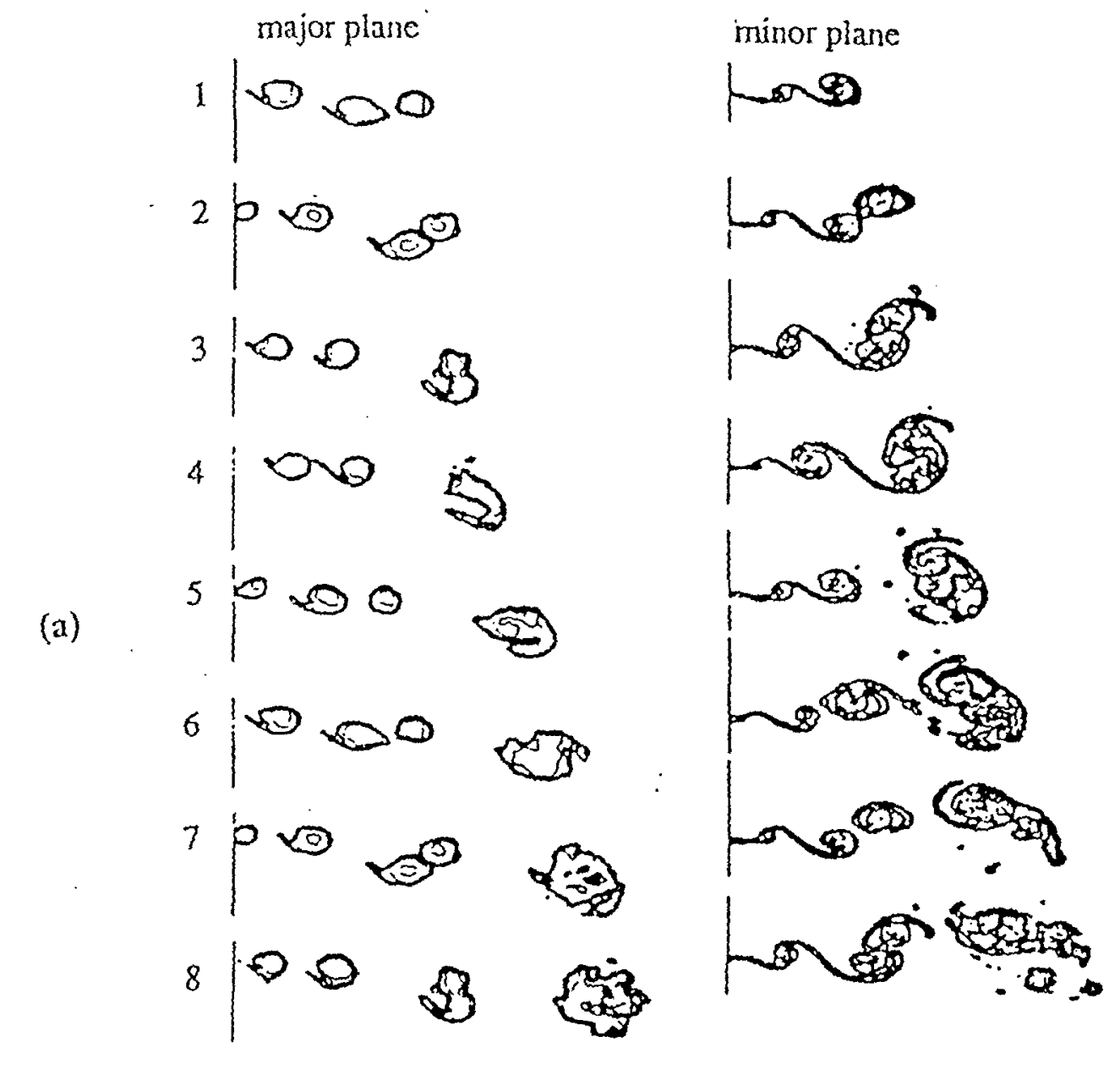

(b)

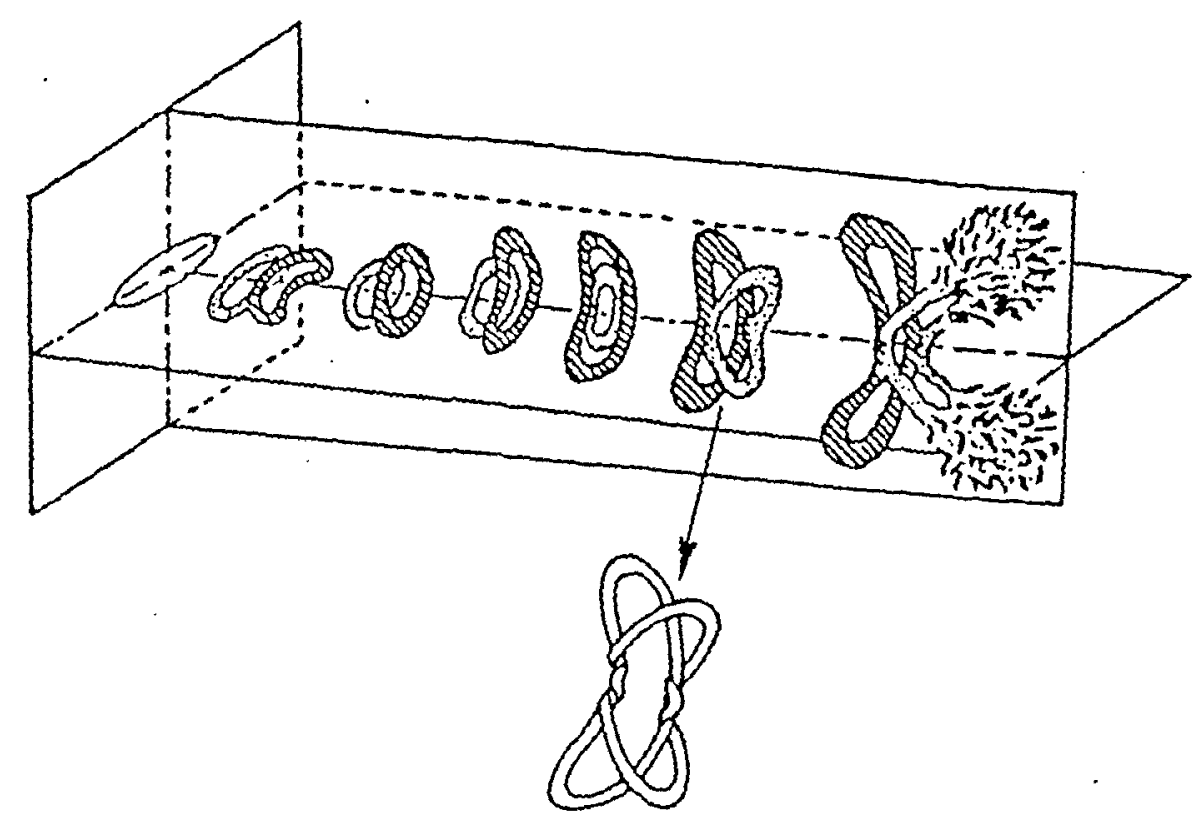

Figure 7 (a) Sequence of flow visualization pictures in the major and minor planes during pairing, presented in the forn of isointensity contours.(b) Schematic of the vortex evolution process during pairing. 
miljor plane

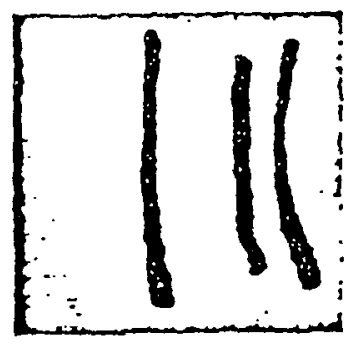

(c)
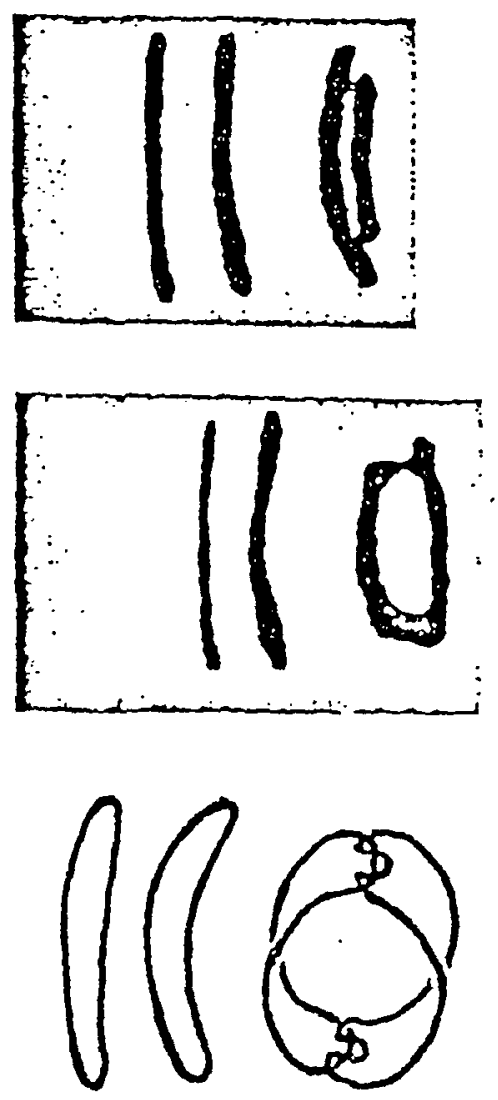

minor plane
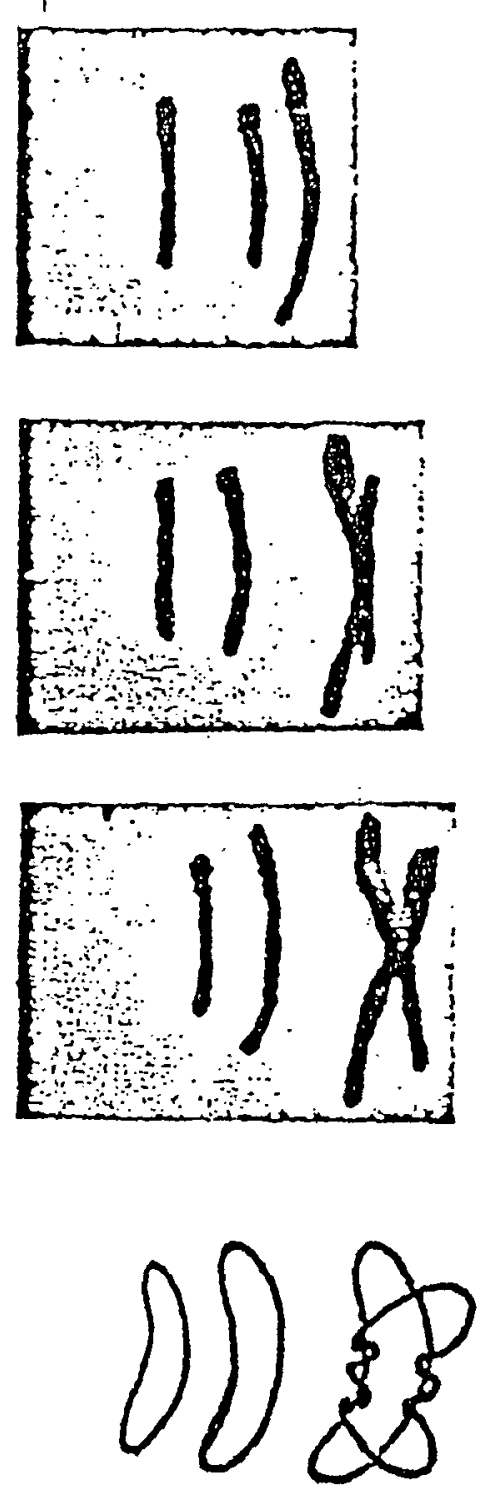

(d)

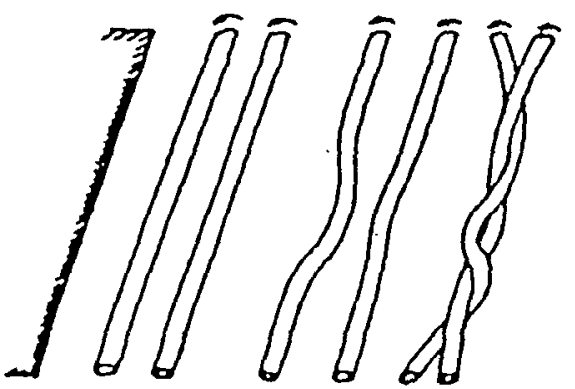

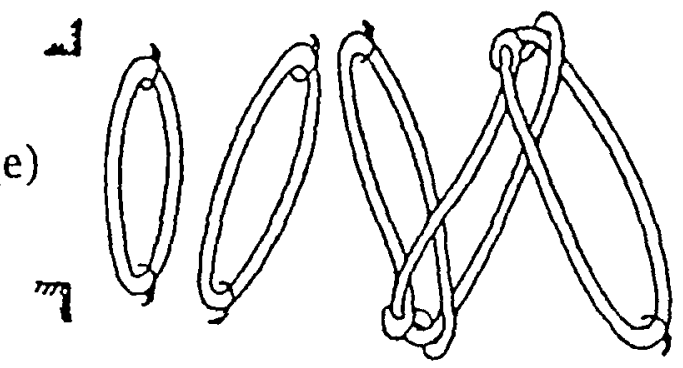

Figure 7 (c) Flow visualization pictures taken simultaneously in the major and minor planes during pairing.

Schematics of the entanglement of vortices in: (d) plane jet; (e) circular jet. 

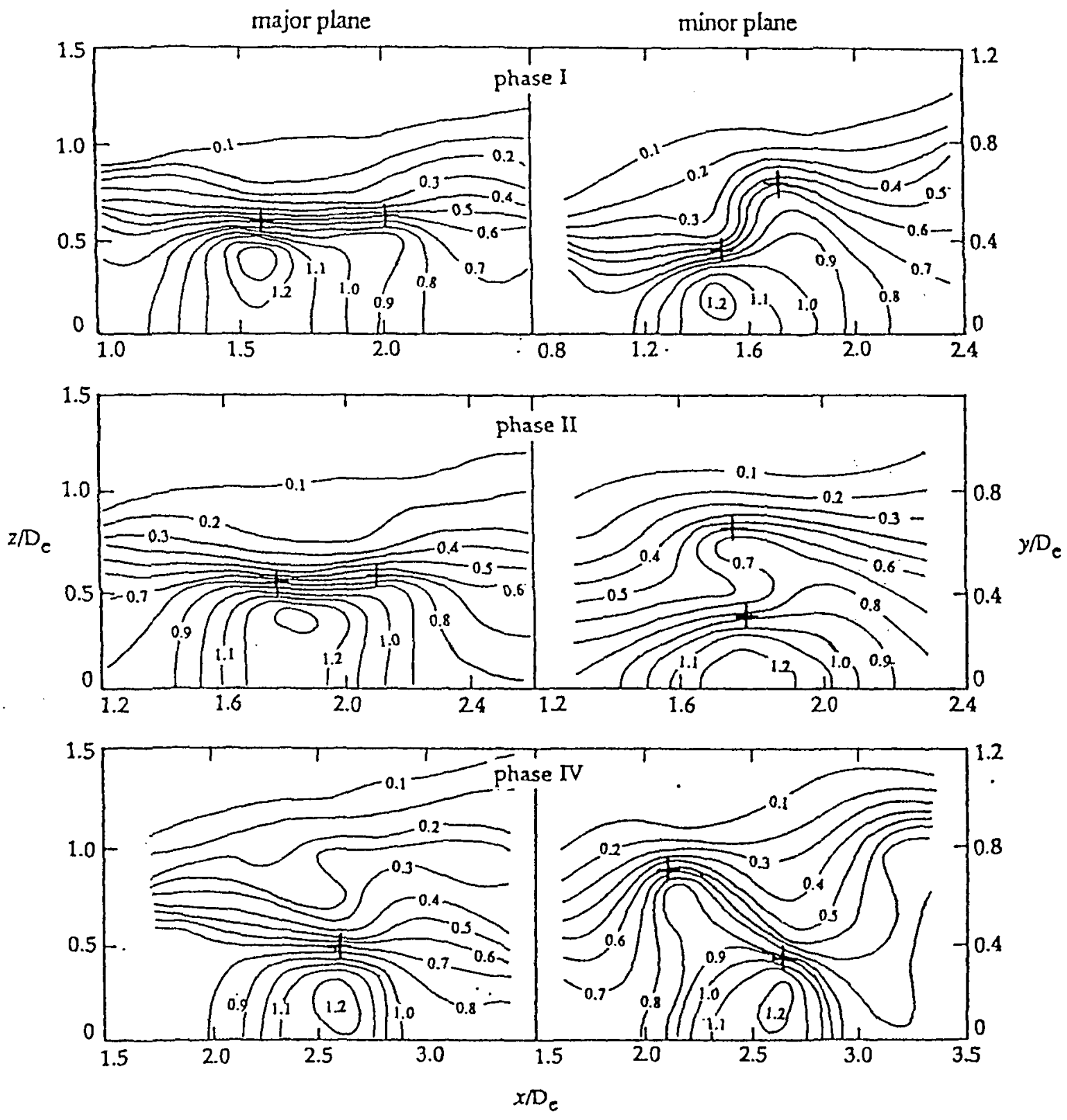

Figure 8 Contours of phase-average longitudinal velocity $\left\langle u>N_{\mathrm{e}}\right.$. 

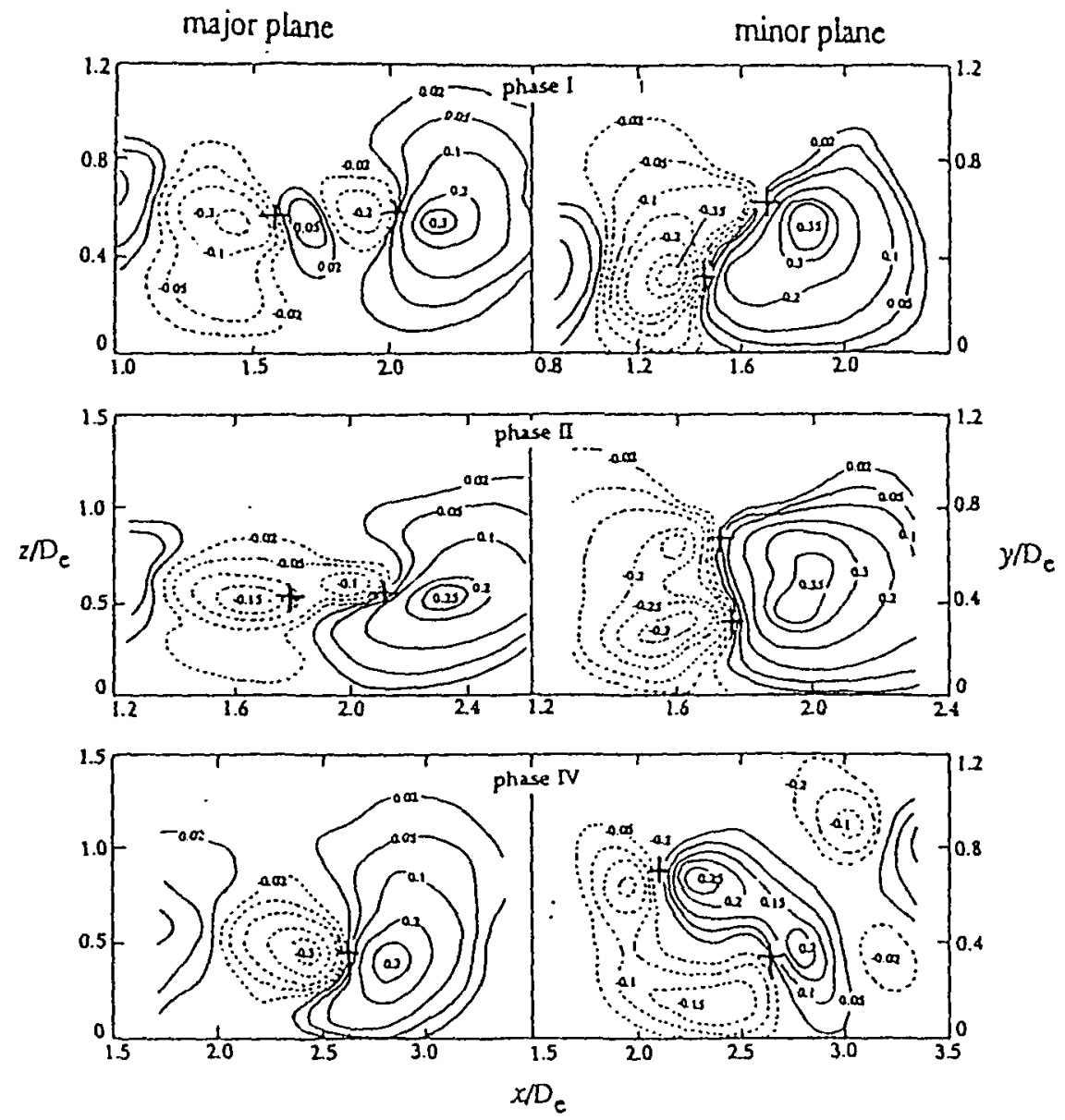

Figure 9 Contours of phase-average transverse velocity $\langle v\rangle / \mathrm{U}_{\mathrm{e}}$

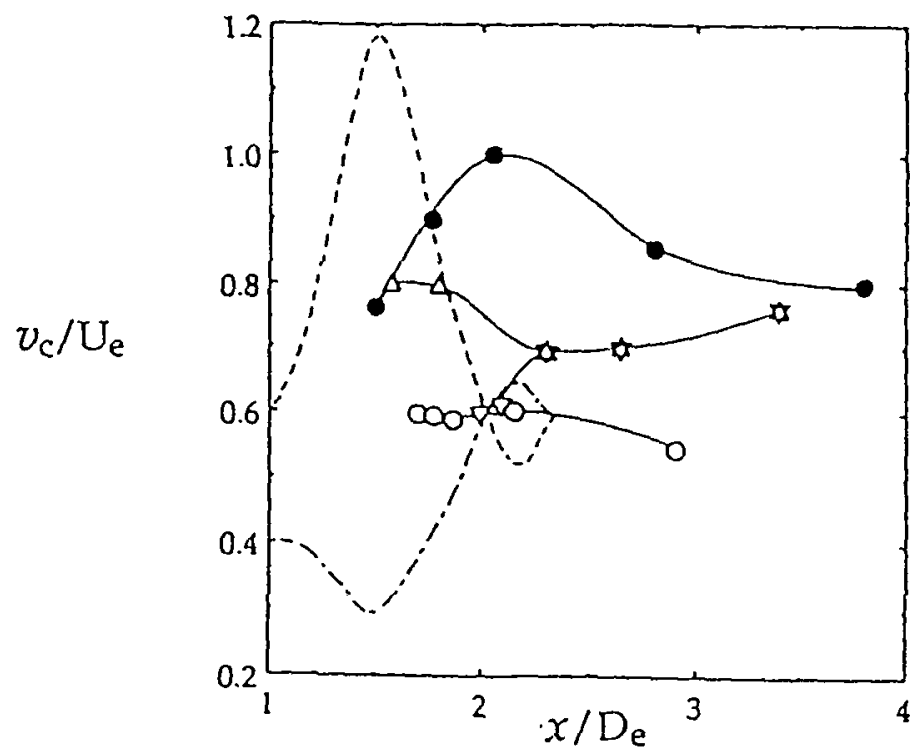

Figure 10 Convection velocities of vortices during pairing. 0 , leading vortex (minor plane); -, trailing vortex, (minor plane); $\nabla$, leading vortex (major plane); $\Delta$, trailing vortex (major plane); - - -, trailing vortex (circular jet); _ _ _ leading vortex (circular jet). . 
major plane

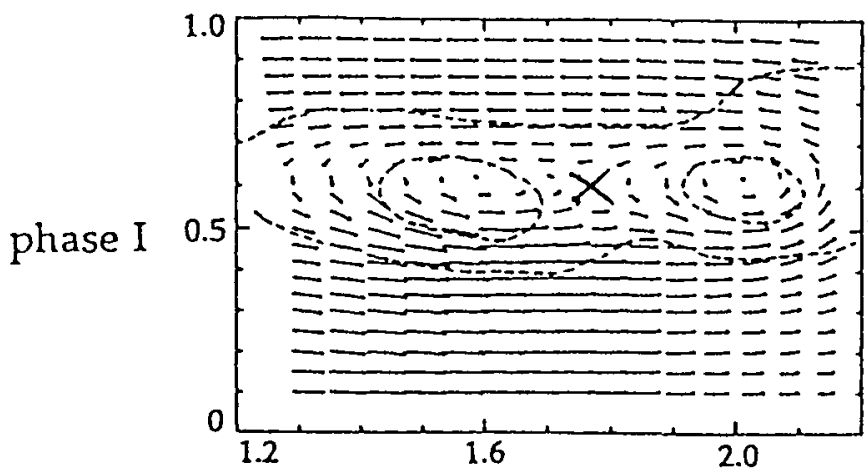

minor plane
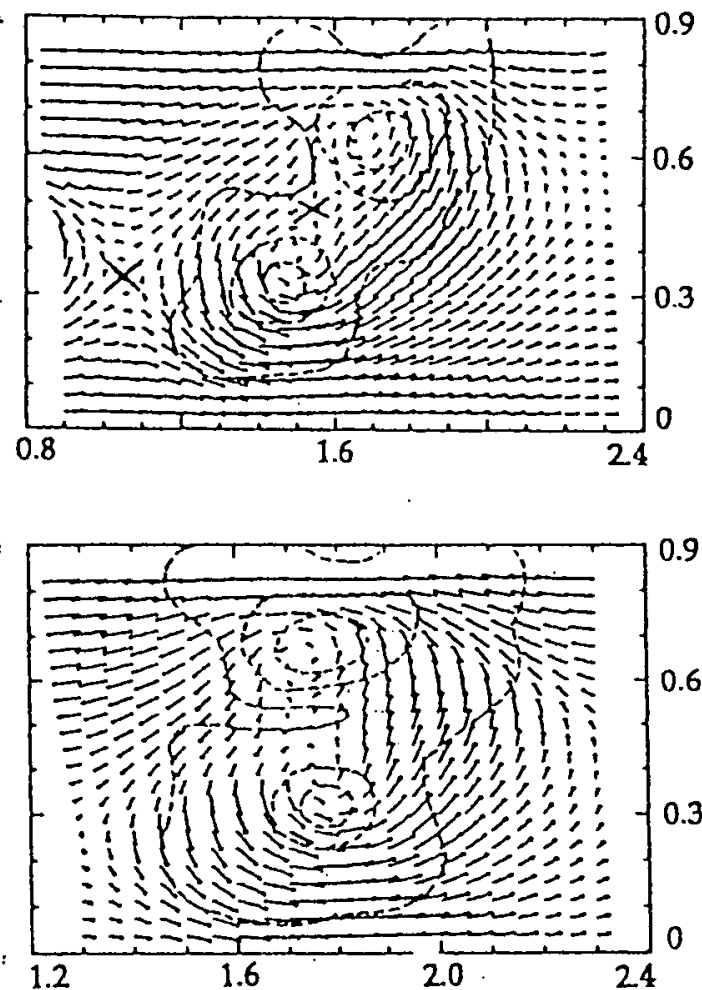

$y / \mathrm{D}_{\mathrm{e}}$
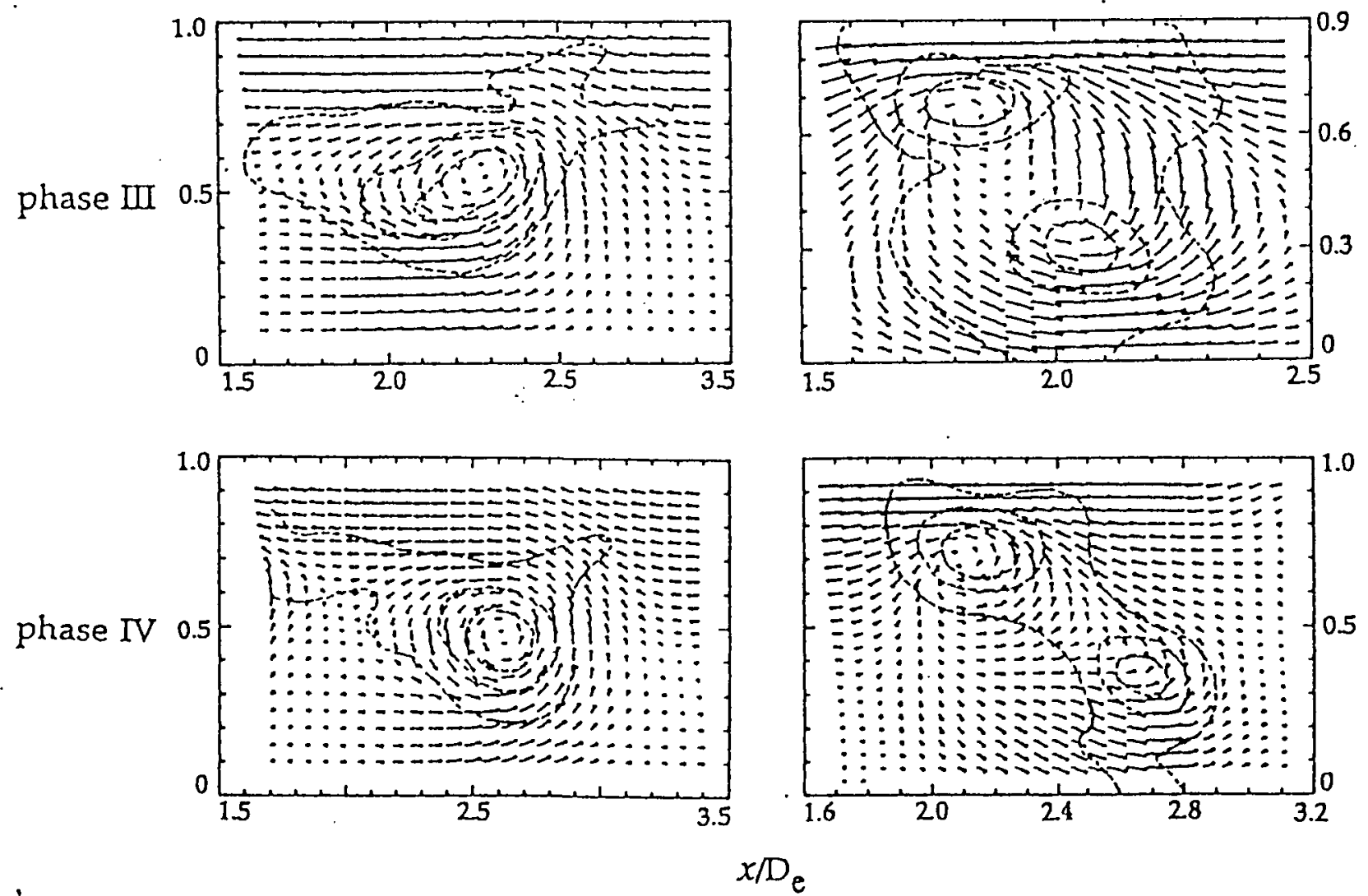

Figure 11 Distributions of phase-average velocity vectors during pairing. 

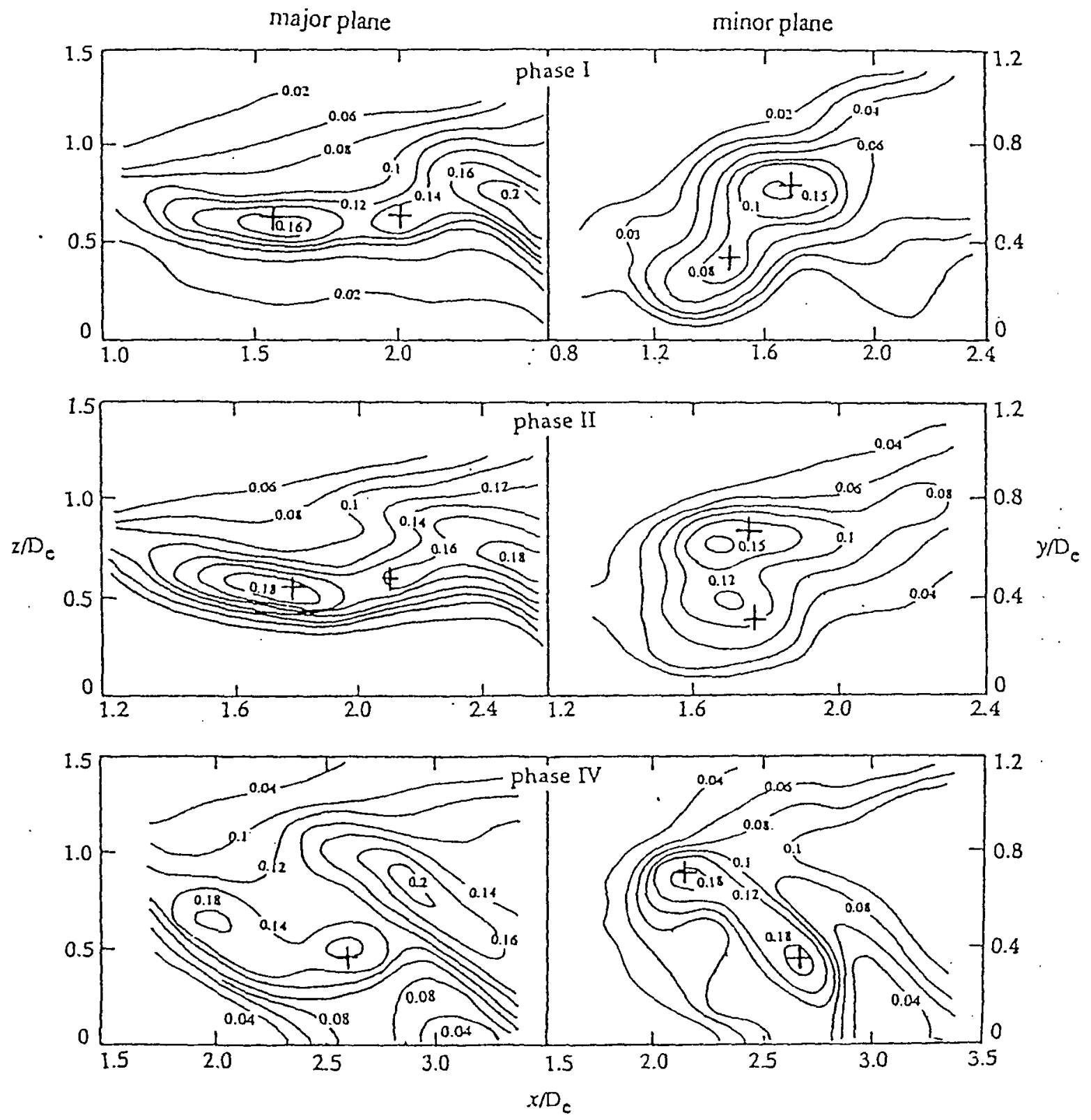

Figure 12 Contours of incoherent longitudinal turbulence intensity $\left\langle u_{\mathrm{r}}{ }^{2}>1 / 2 / \mathrm{U}_{\mathrm{e}}\right.$. 

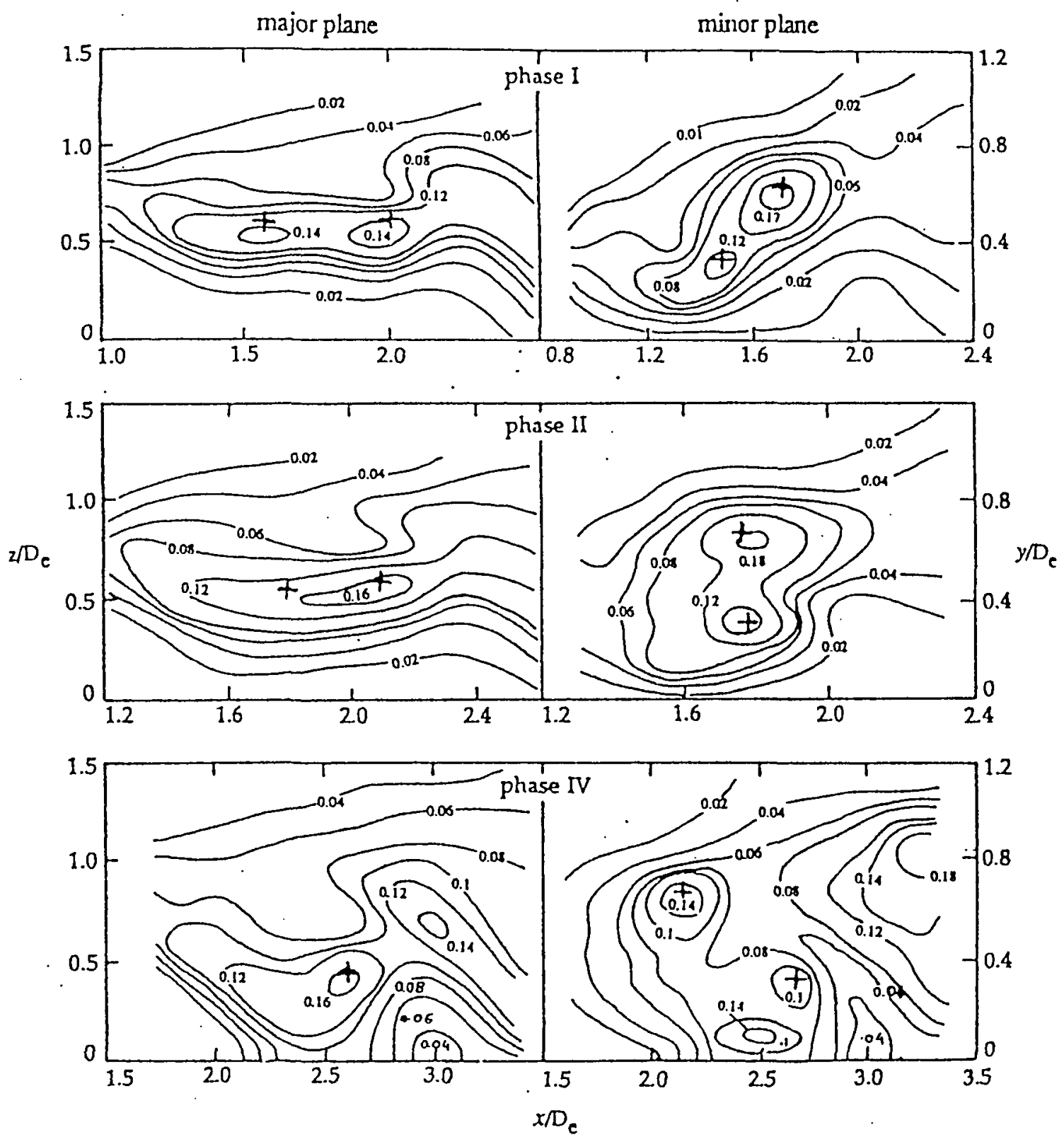

Figure 13 Contours of incoherent transverse turbulence intensity $\left\langle v_{\mathrm{r}}{ }^{2}\right\rangle 1 / 2 / \mathrm{U}_{\mathrm{e}}$. 

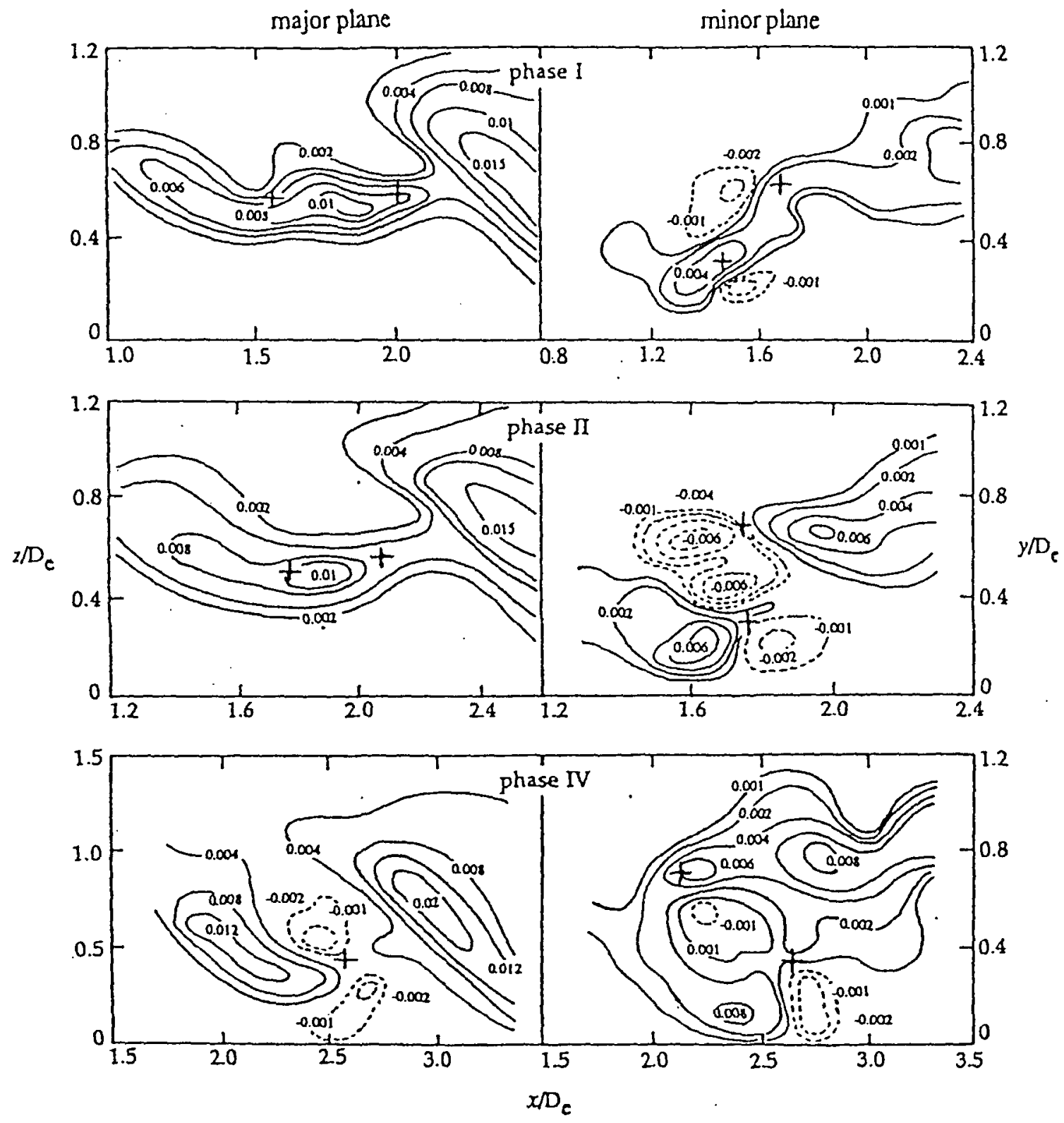

Figure 14 Contours of incoherent Reynolds stress $\left\langle u_{\mathrm{r}} v_{\mathrm{r}}\right\rangle / \mathrm{U}_{\mathrm{e}}{ }^{2}$. 
(a)

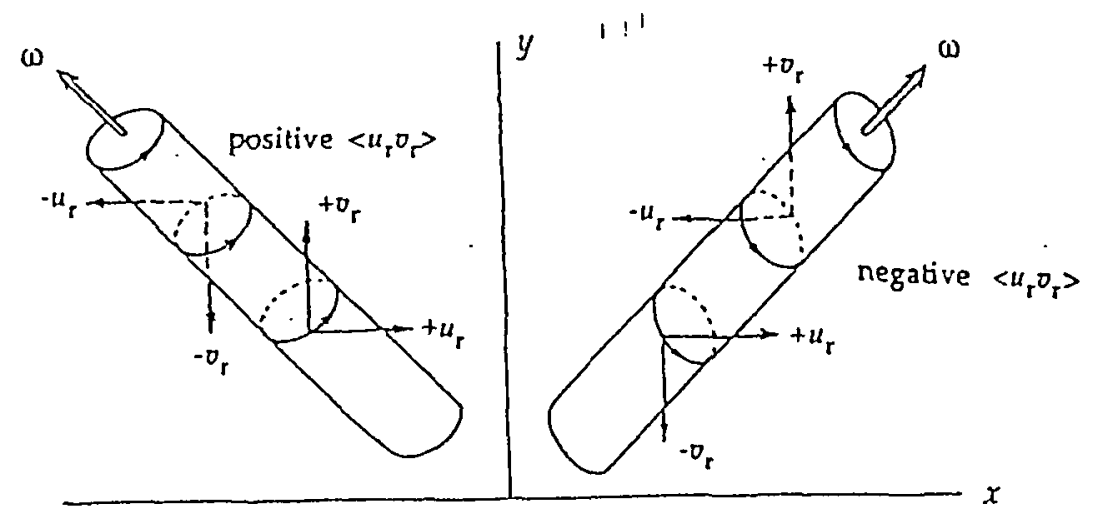

(b)
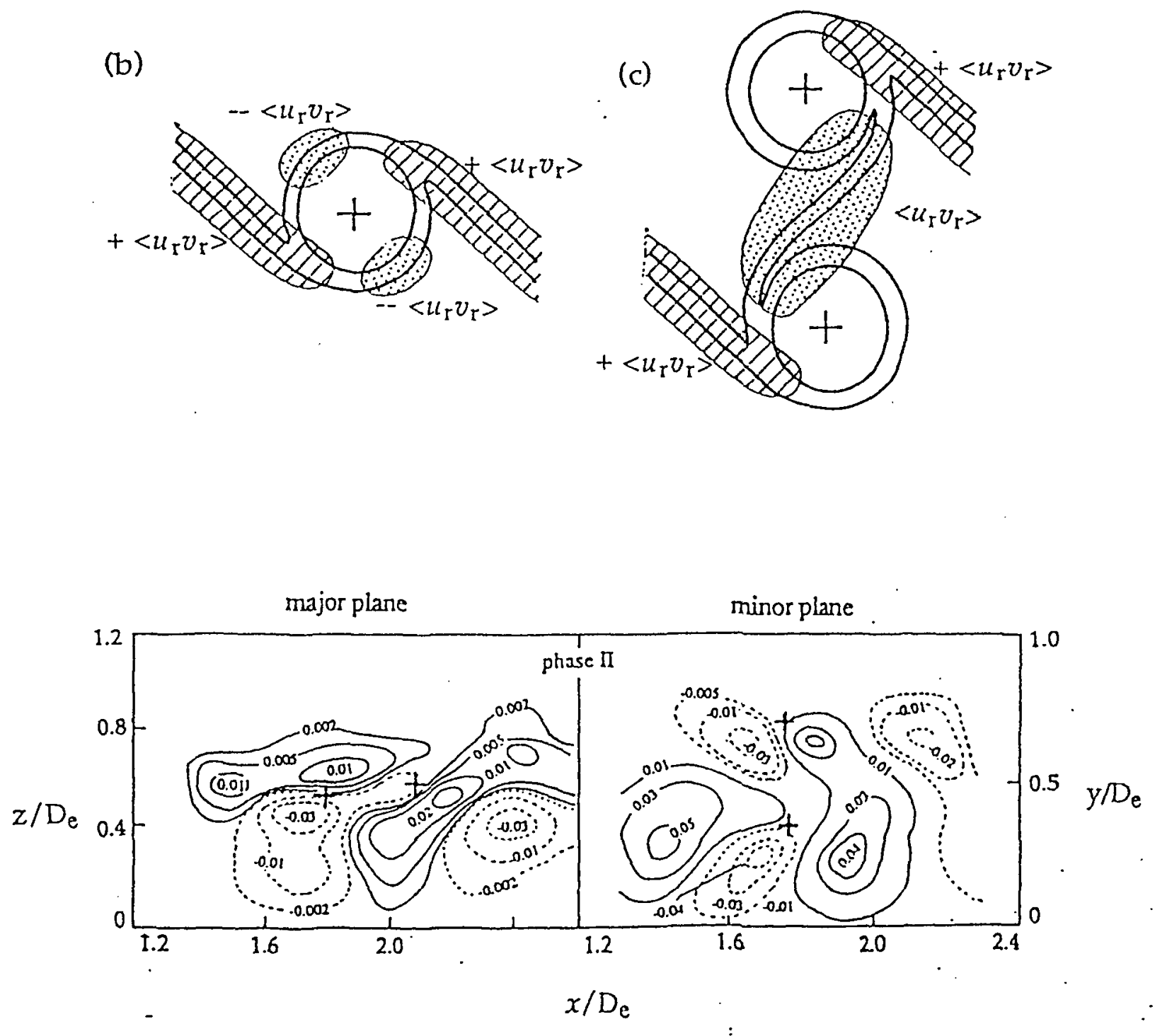

Figure 15 (a) Schematics showing the mechanism of positive and negative $\left\langle u_{\mathrm{T}} v_{\mathrm{r}}\right\rangle$ generation. (b, c) Schematics showing the regions of positive and negative $\left\langle u_{\mathrm{T}} v_{\mathrm{r}}\right\rangle$ (d) Contours of coherent Reynolds stress $\left\langle u_{\mathrm{p}} v_{\mathrm{p}}>\mathrm{U}_{\mathrm{e}}{ }^{2}\right.$ 

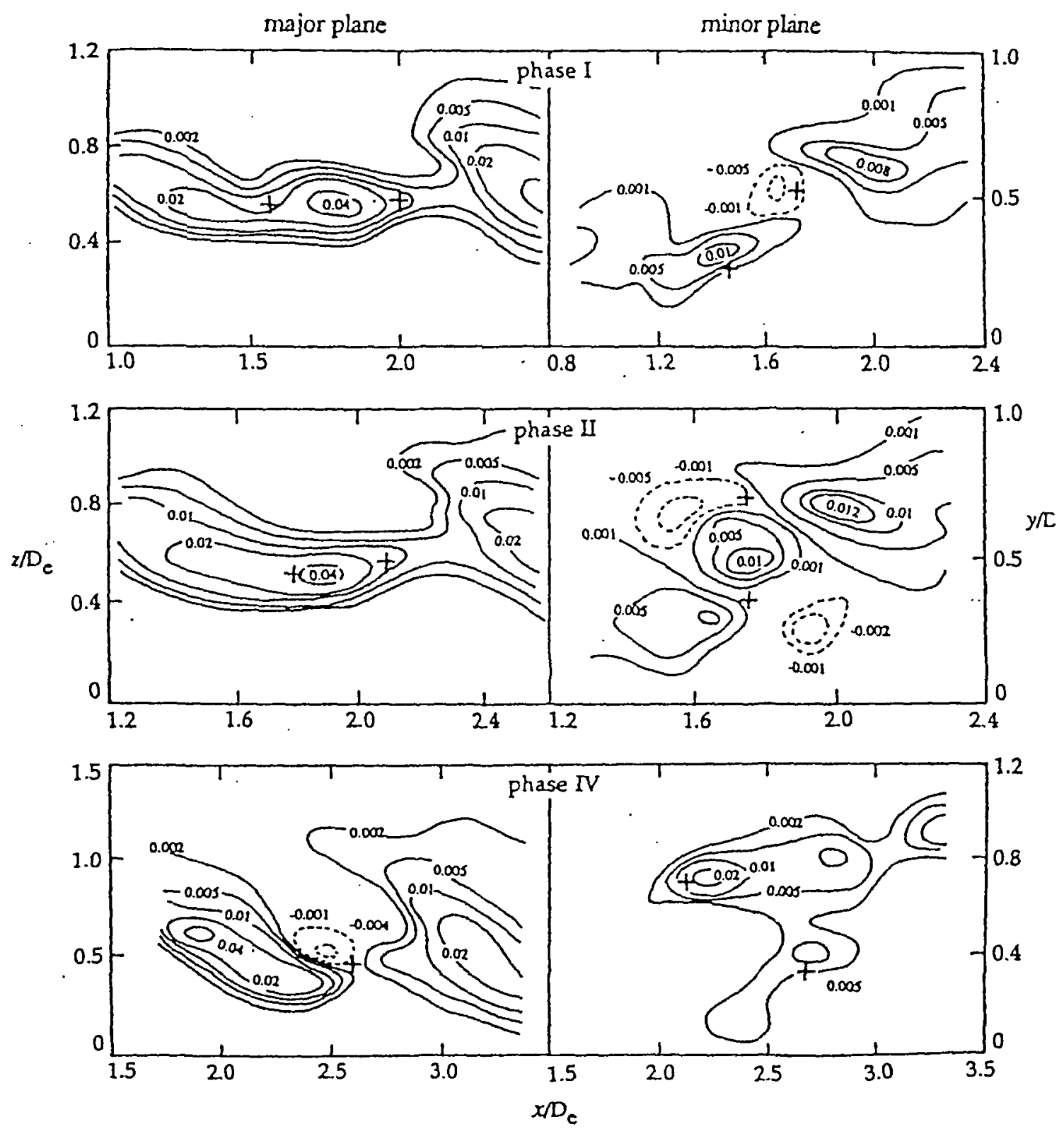

Figure 16 (a) Contours of coherent shear production $\left\langle\mathrm{P}_{s}\right\rangle /\left(\mathrm{U}_{\mathrm{e}}^{2} . \mathrm{f}\right)$. 

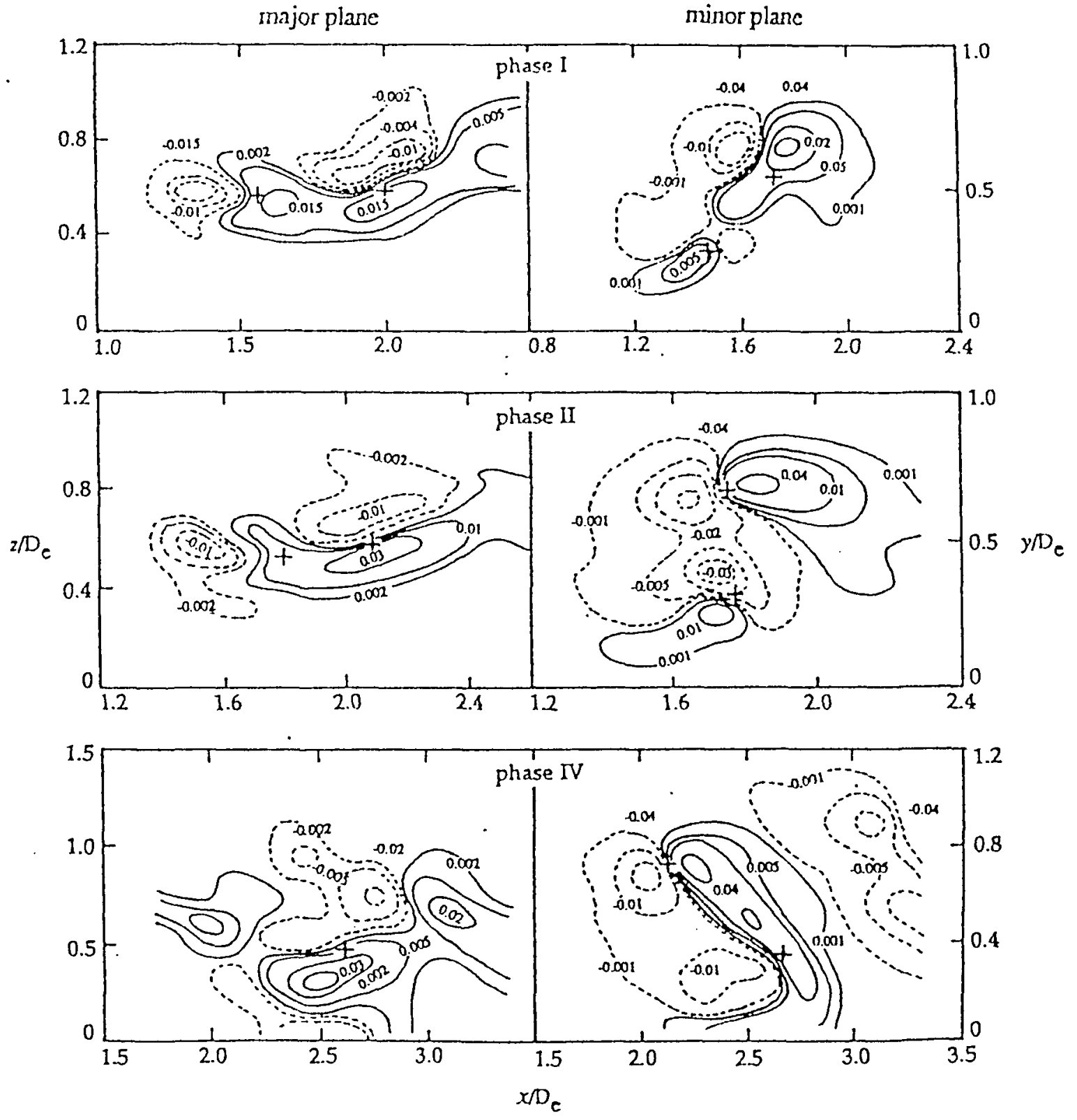

Figure 16 (b) Contours of coherent normal production $\left\langle P_{n}>/ U_{e}^{2} . f\right)$ 

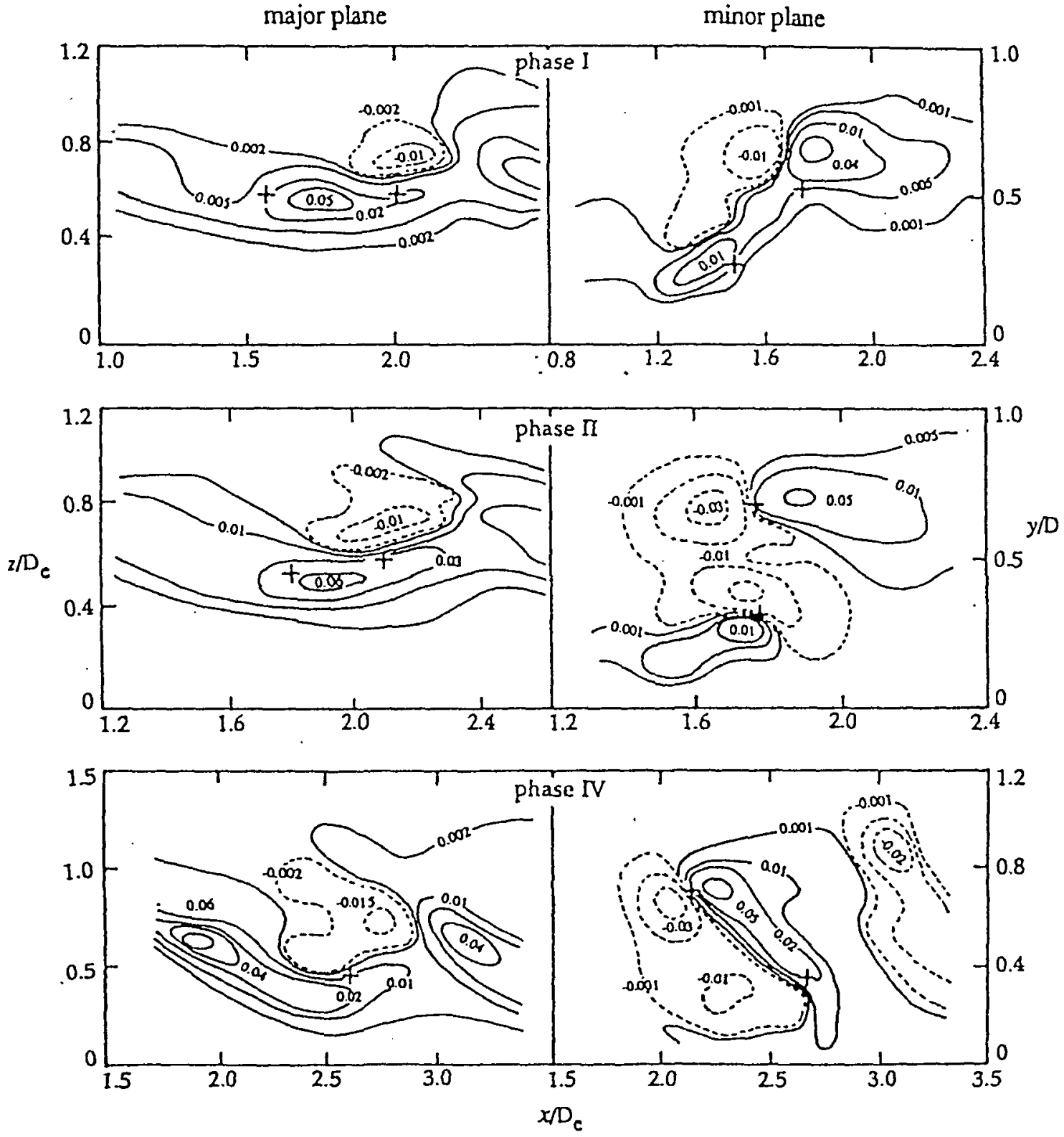

Figure 16 (c) Contours of total coherent production $\left\langle\mathrm{P}>/ \mathrm{U}_{\mathrm{e}}^{2}\right.$.f 
(a)
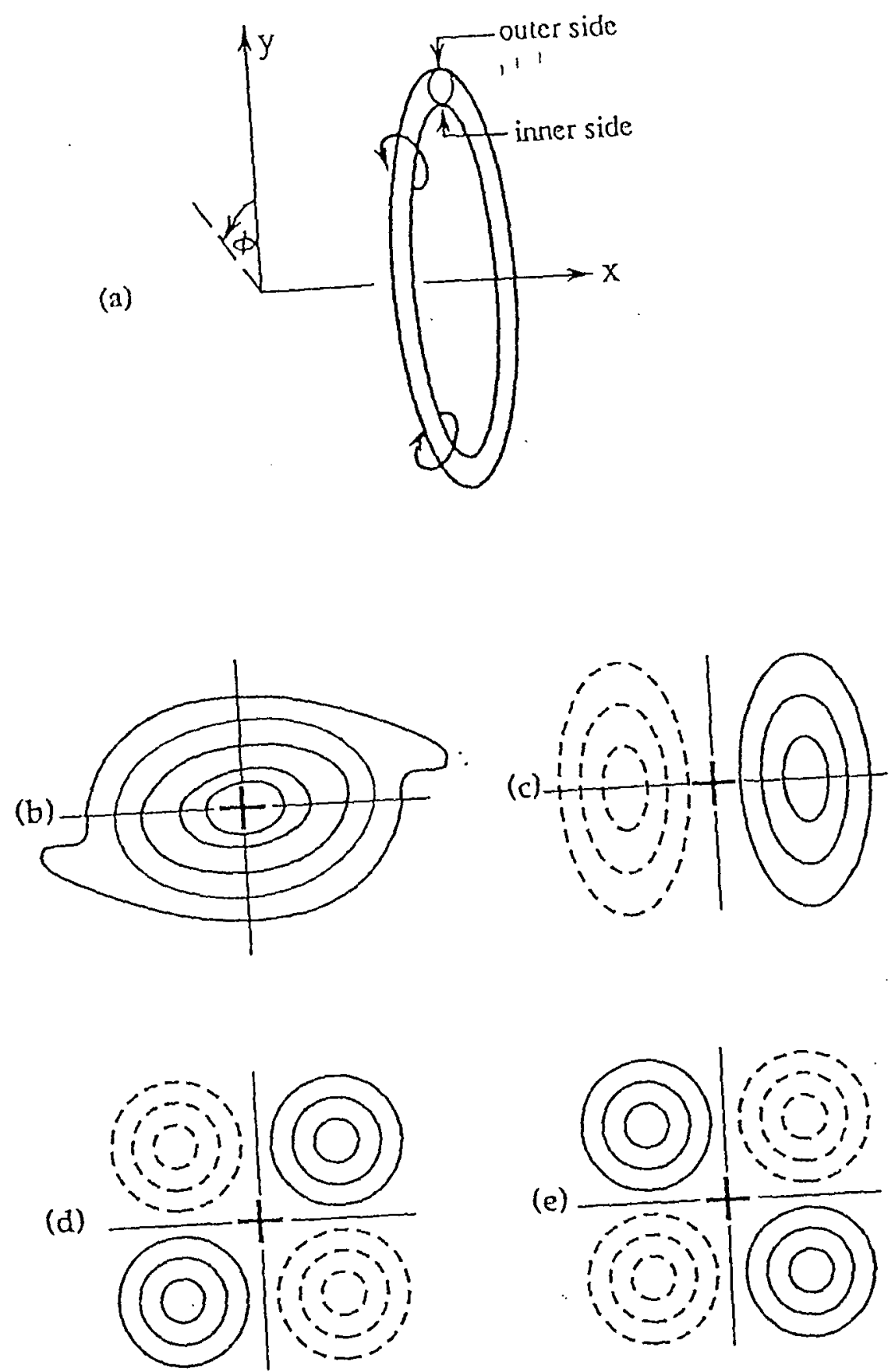

(f)

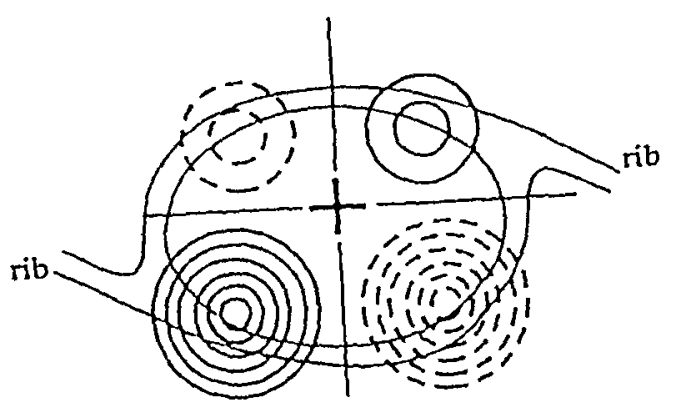

Figure 17 (a) Schematic of a ring vortex and cylindrical coordinates. Qualitative contours of: (b)

dimensional Qualitative contours of $\partial<u>\mid \partial x$, showing the effects of structure curvature. 
(a)

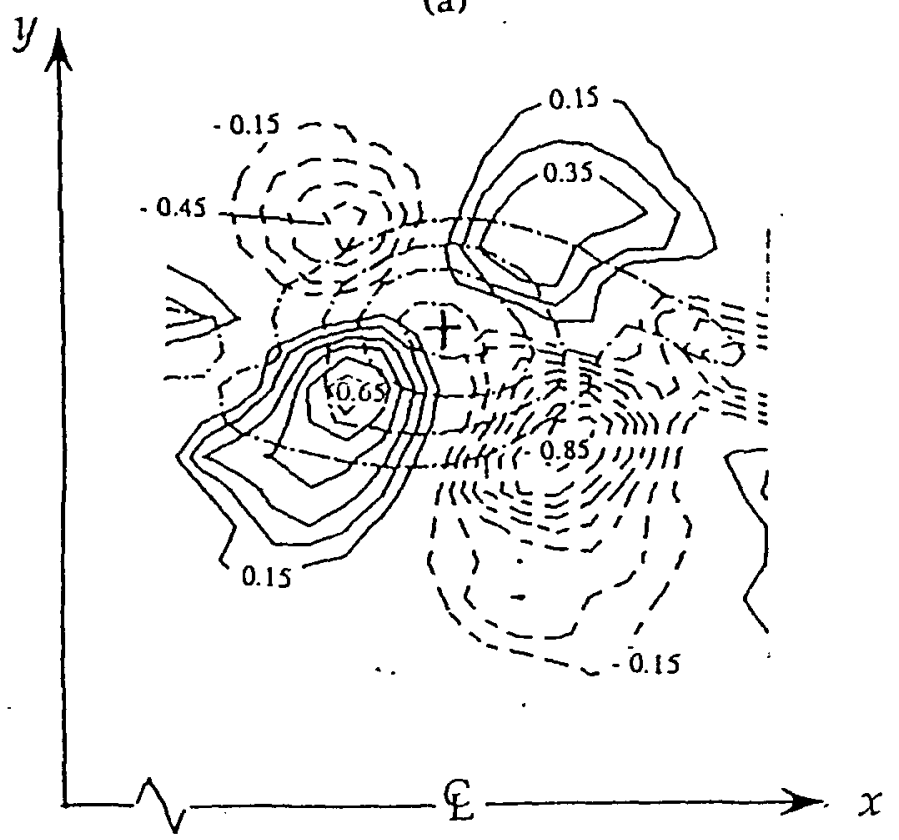

(b)

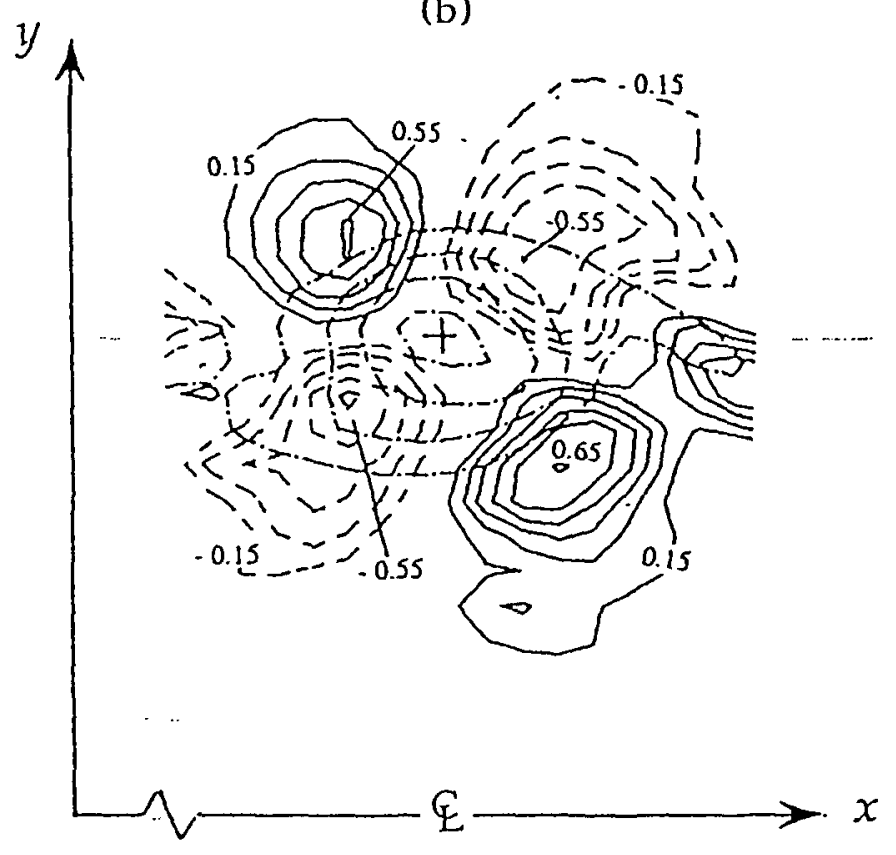

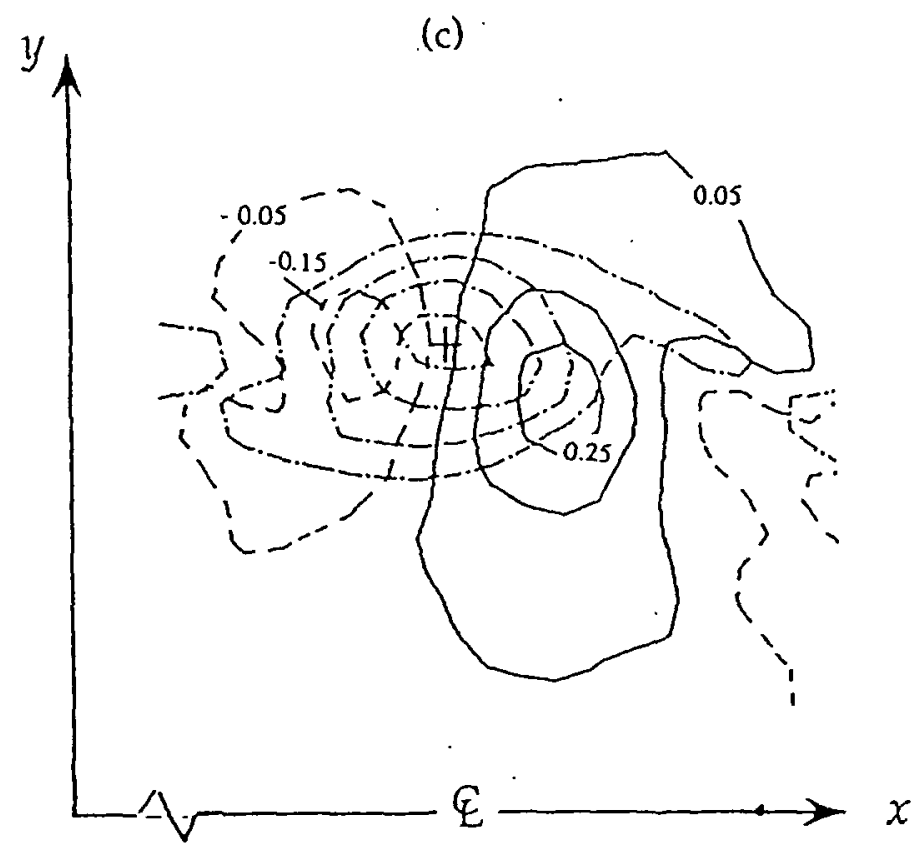

Figure 18 Numerical results of a circular jet.showing contours of: (a) $\partial\langle u\rangle / \partial x$; (b) $\partial\langle v\rangle / \partial y$; (c) $\langle v>/ y$. Solid and dashed lines are contours of positive and negative values respectively. _- _ voricity contours. 
(a)

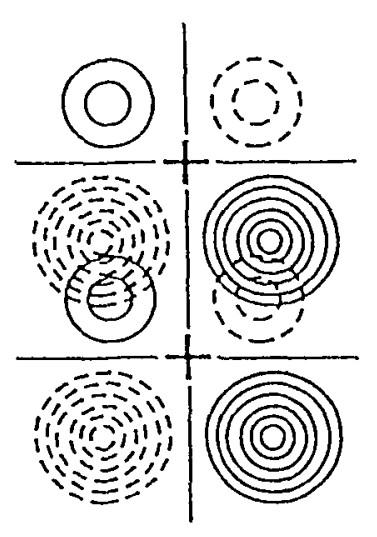

$\left(-\frac{\partial<u>}{\partial x}\right)_{\text {vortex } 1}+\left(-\frac{\partial<u>}{\partial x}\right)_{\text {vortex } 2}$

(e)

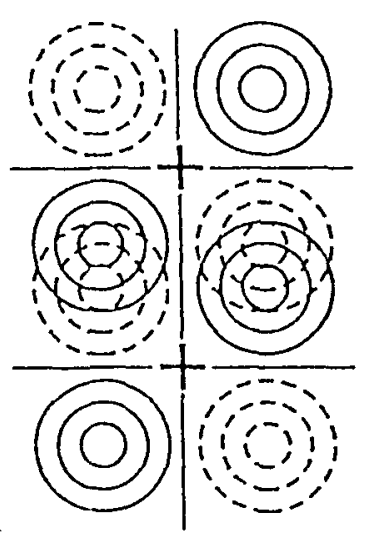

$\left(-\frac{\partial<v>}{\partial y}\right)_{\text {vortex } 1}+\left(-\frac{\partial<v>}{\partial y}\right)_{\text {vortex } 2}$ (b)

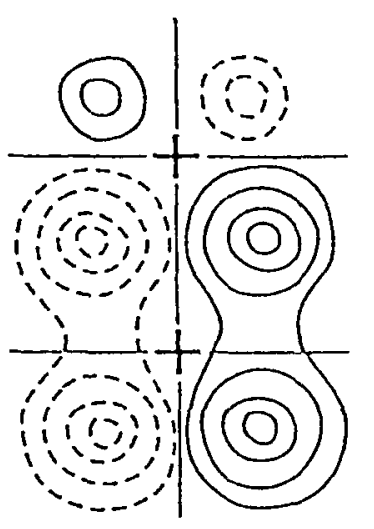

$-\frac{\partial<u>}{\partial x}$

(f)

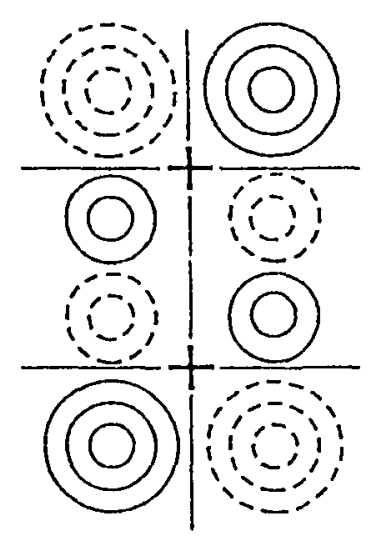

$-\frac{\partial<0>}{\partial y}$ (c)

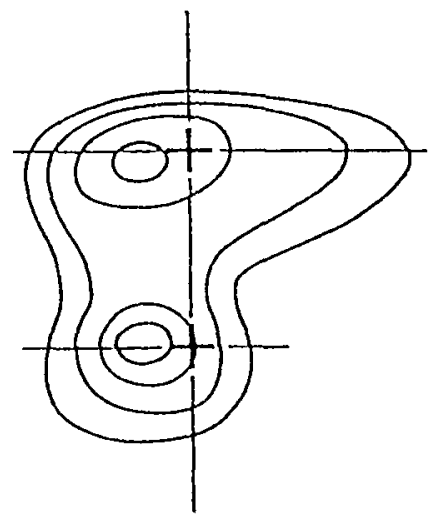

$\left\langle u_{\mathrm{r}}^{2}\right\rangle$ (d)

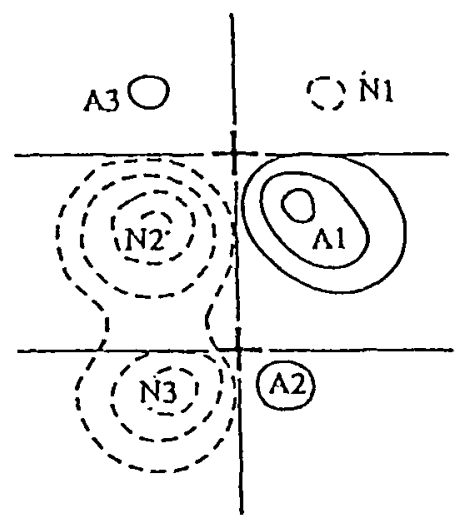

$\left\langle P_{n u}\right\rangle=-\left\langle u_{r}{ }^{2}\right\rangle \frac{\partial\langle u\rangle}{\partial x}$ (g)

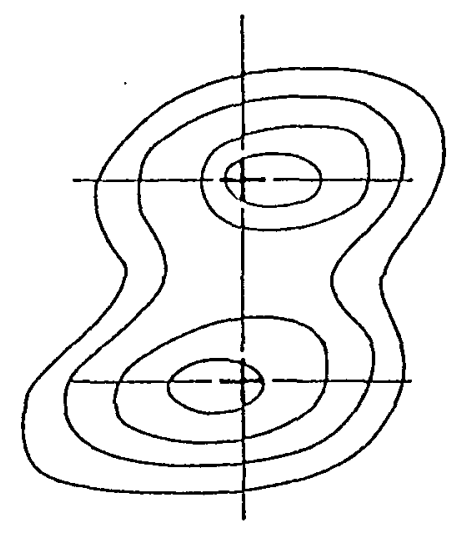

$\left\langle 0_{\mathrm{r}}{ }^{2}\right\rangle$ (h)

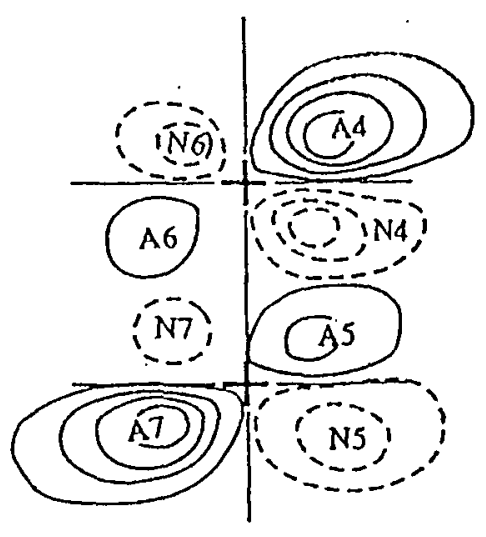

$\left\langle P_{n v}\right\rangle=-\left\langle v_{r}^{2}\right\rangle \frac{\partial<\partial\rangle}{\partial y}$

Figure 19 (a) Qualitative contours of interacting vortices at phase II: (a) superposition of $-\partial\langle u>\partial x$ of vortex 1 and vortex 2 ; (b) $-\partial\langle u\rangle \partial x$; (c) $\left\langle u_{\mathrm{T}}^{2}\right\rangle$; (d) $\left\langle\mathrm{P}_{n u}>\right.$; (e) superposition of $-\partial<v>/ \partial y$ of vortex 1 and vortex 2 ; (f) $-\partial<v>/ \partial y$; (g) $\left\langle v_{\mathrm{r}}{ }^{2}>\right.$; (h) $\left\langle\mathrm{P}_{\mathrm{nv}}>\right.$. 
(i)

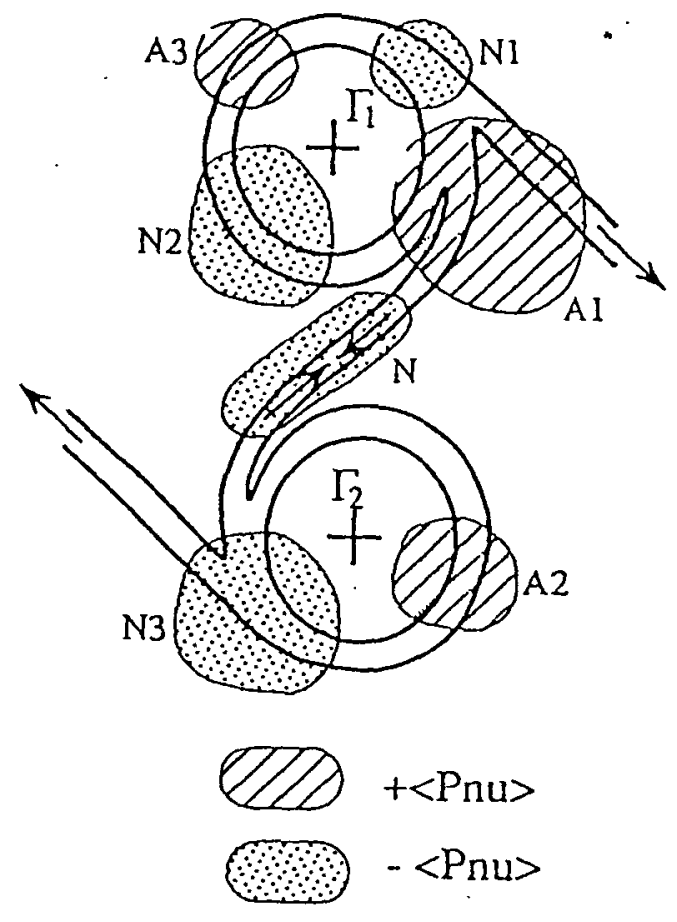

(j)

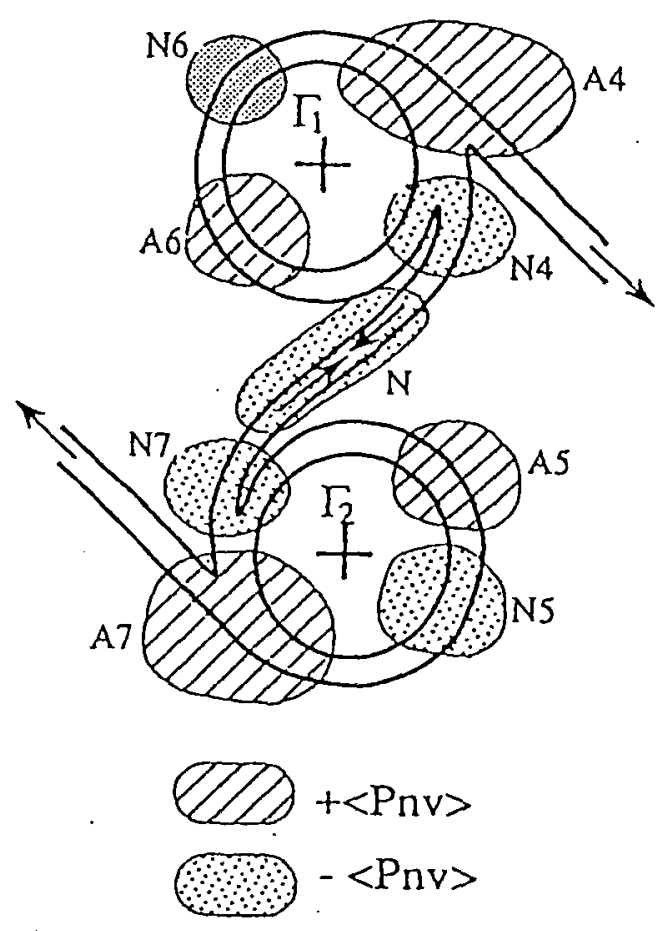

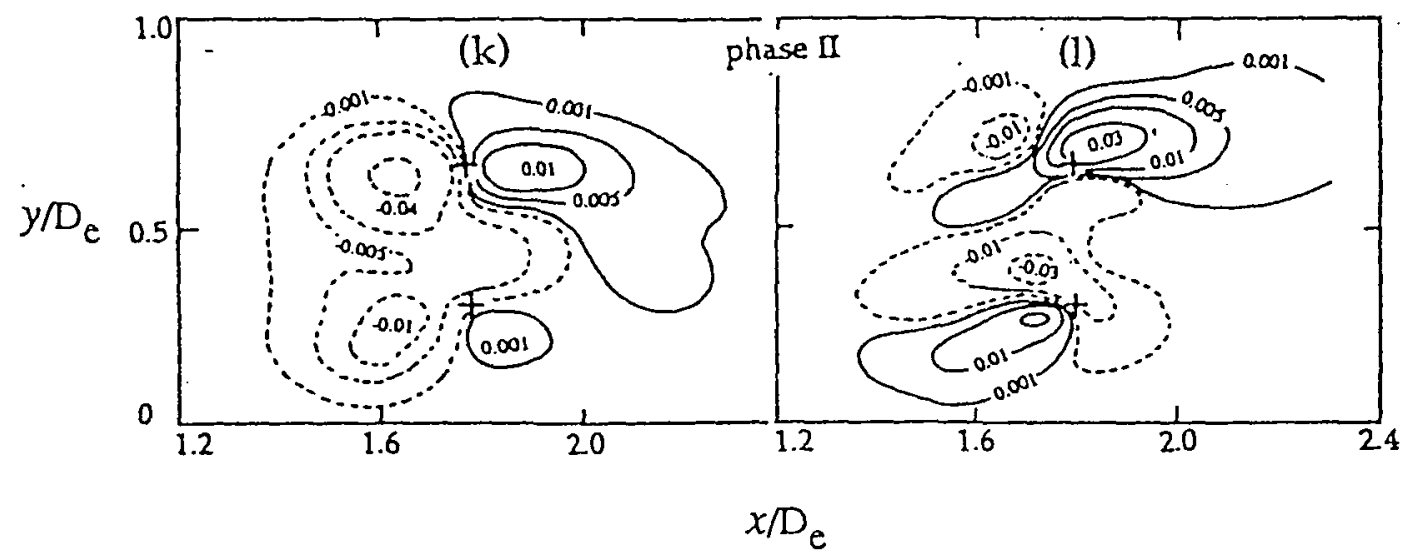

Figure 19 Schematics showing how rib-roll interaction produce regions of: (i) $\left\langle P_{n u}\right\rangle$; (j) $\left\langle P_{n v}\right\rangle$. (k) Contours of the normal production term $<\mathrm{P}_{n u}>/\left(\mathrm{U}_{\mathrm{e}}^{2} . \mathrm{f}\right)$; phase II, minor plane. (l) Contours of the normal production term $\left\langle\mathrm{P}_{n v}>/\left(\mathrm{U}_{\mathrm{e}}^{2} . \mathrm{f}\right)\right.$; phase II, minor plane. 

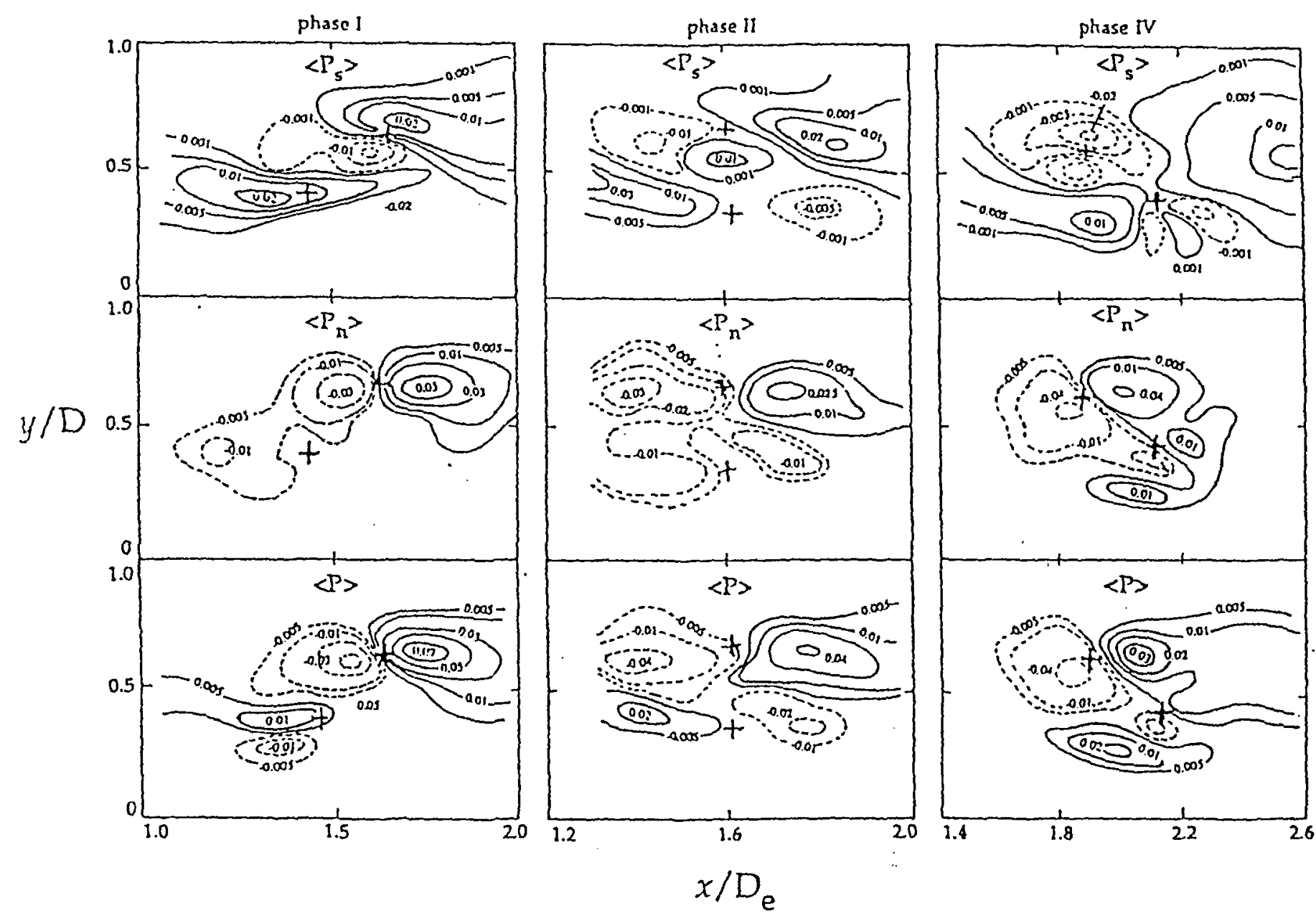

Figure 20 Contours of $\left\langle\mathrm{P}_{\mathrm{s}}>/ \mathrm{U}_{\mathrm{e}}^{2} . \mathrm{f}\right),\left\langle\mathrm{P}_{n}>/ \mathrm{U}_{\mathrm{e}}^{2} . \mathrm{f}\right)$ and $\left\langle\mathrm{P}>/ \mathrm{U}_{\mathrm{e}}^{2}, \mathrm{f}\right)$ during pairing in the circular jet. 
(a)

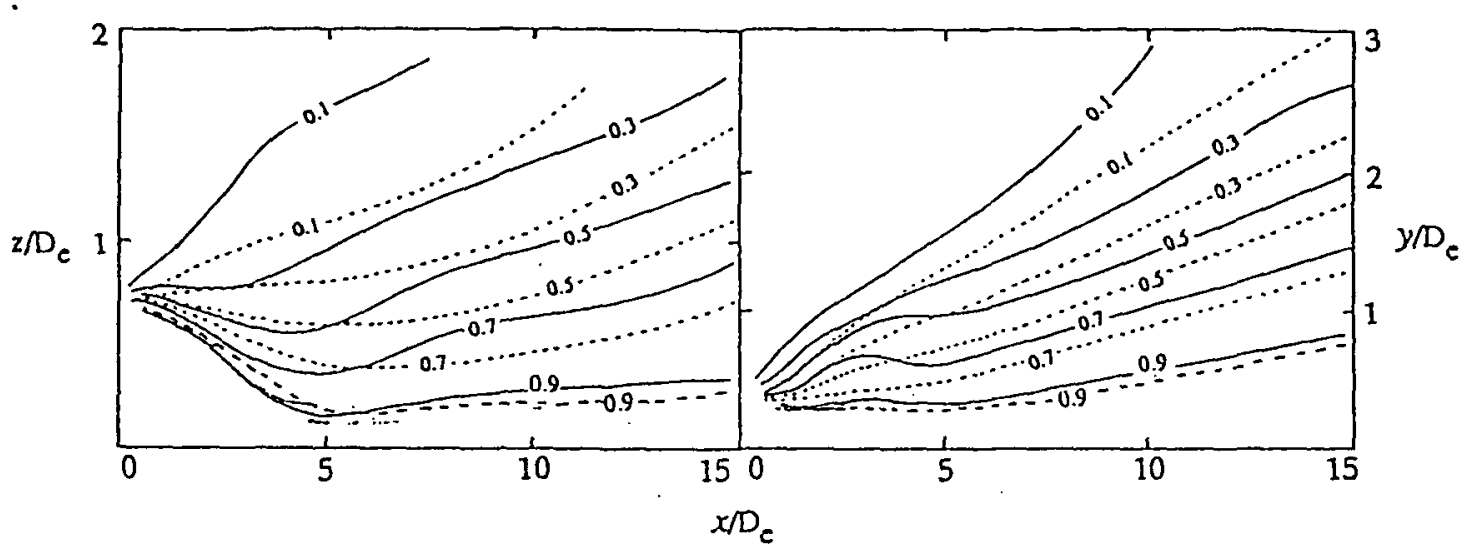

(b)

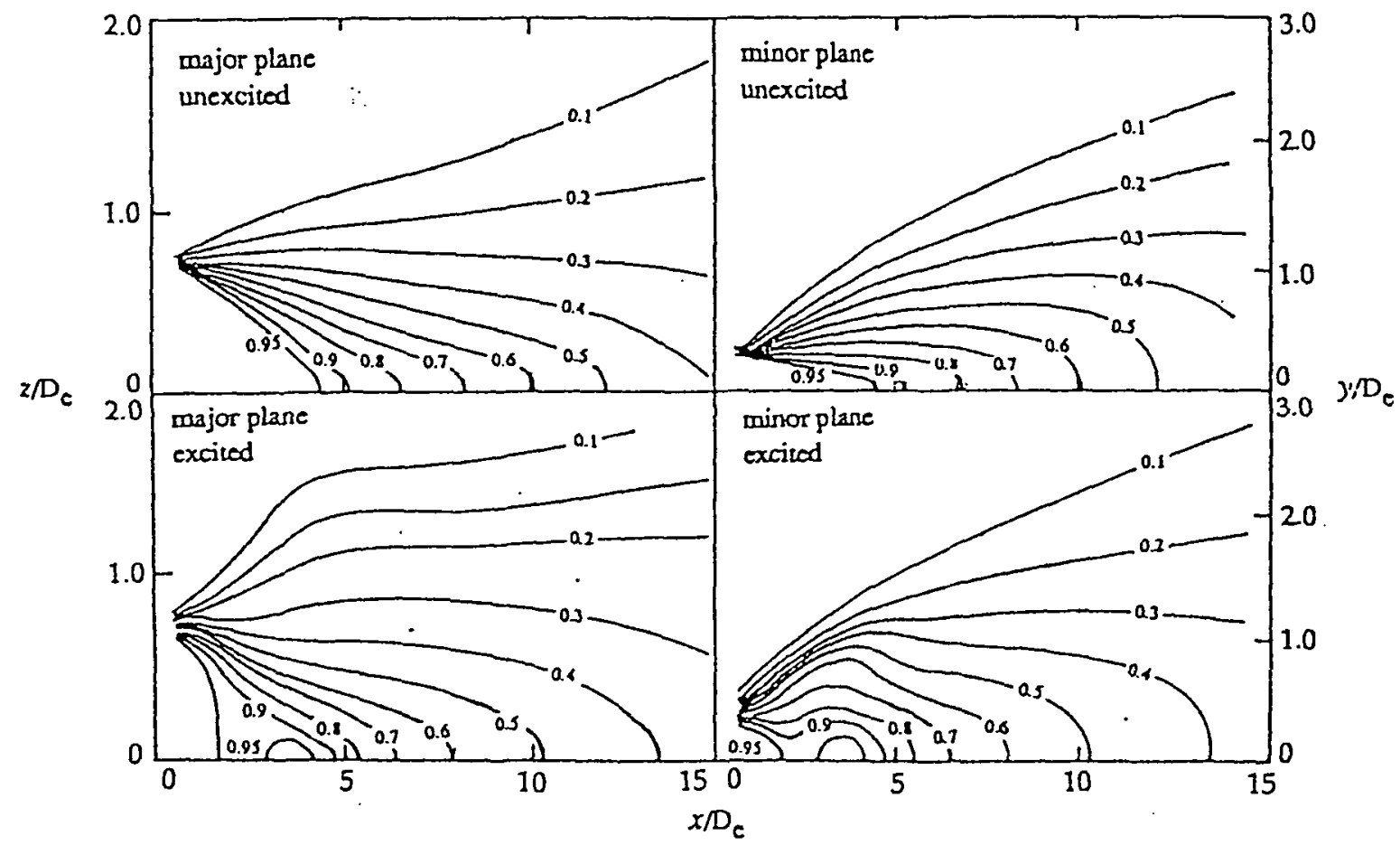

Figure 21 (a) Contours of $U / \mathrm{U}_{\mathrm{c}}$ of the excited $\left(\mathrm{St}_{\mathrm{De}}=0.85\right)$ and unexcited elliptic jets.

- excited; ... unexcited.

(b) Contours of $\mathrm{U} / \mathrm{U}_{\mathrm{e}}$ of the excited ( $\mathrm{S}_{\mathrm{De}}=0.85$ ) and unexcited elliptic jets. 


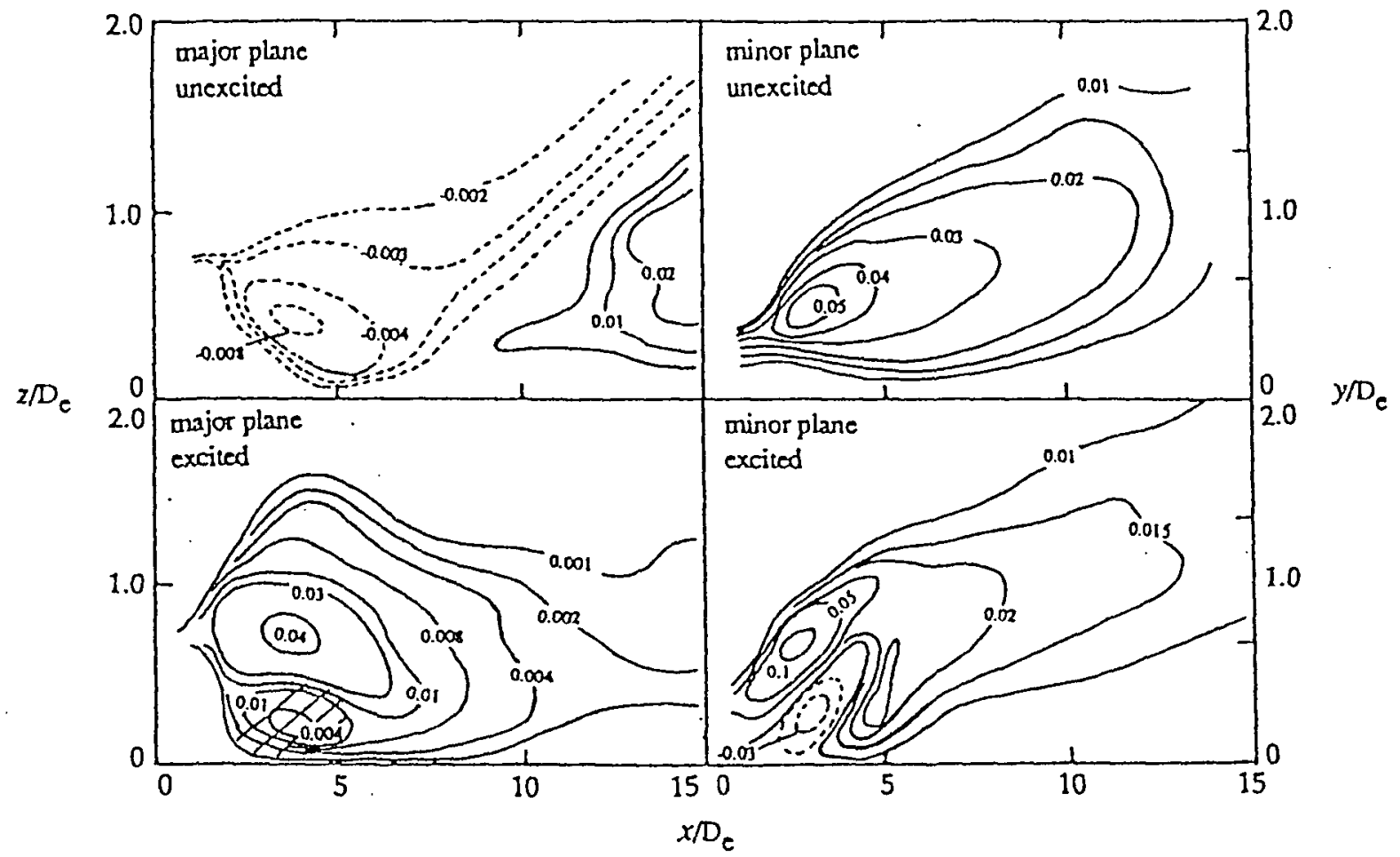

Figure 22 Contours of $\mathrm{V} / \mathrm{U}_{\mathrm{e}}$ of the excited $\left(\mathrm{St}_{\mathrm{De}}=0.85\right.$ ) and unexcited elliptic jets.

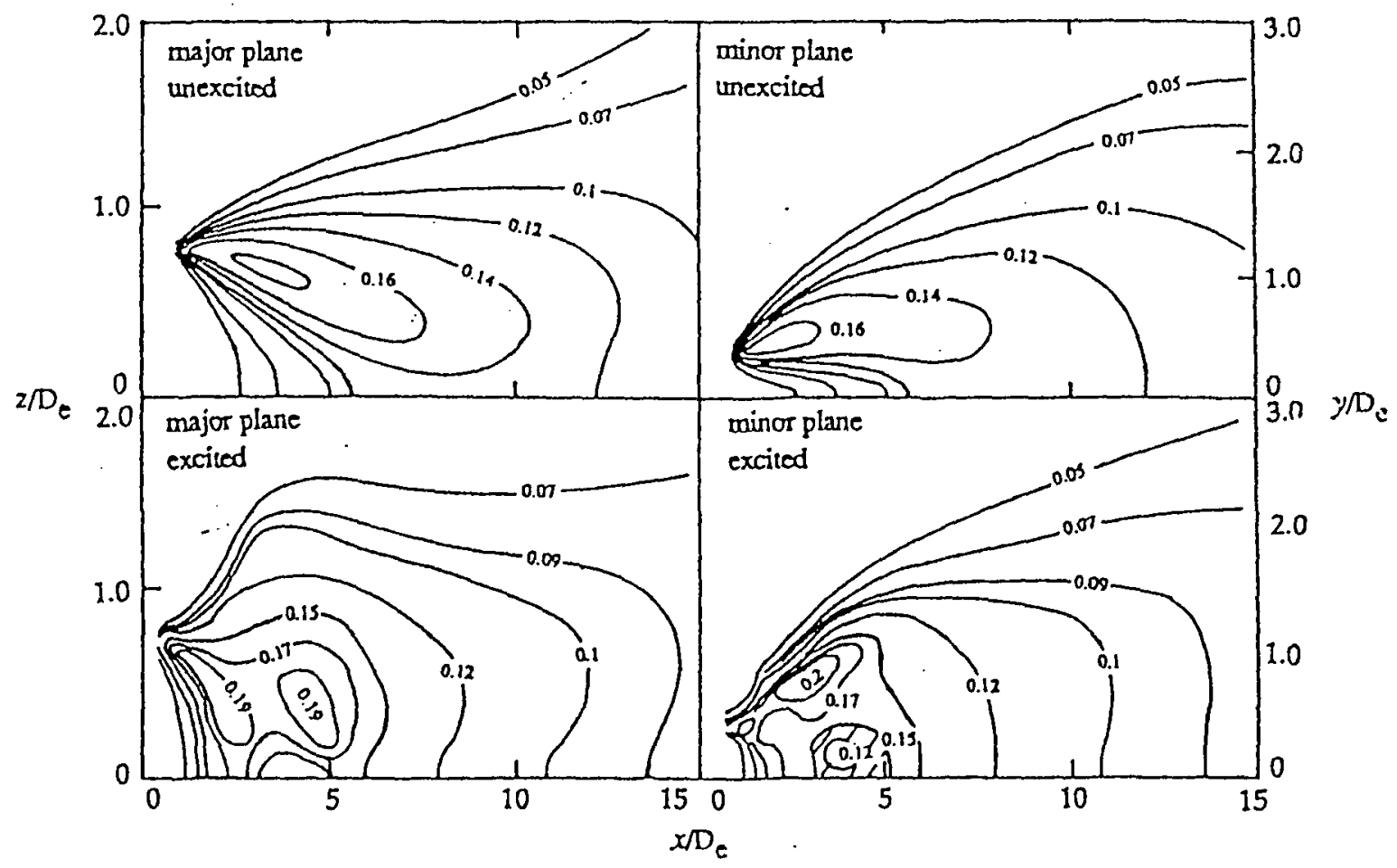

Figure 23 Contours of $u^{\prime} / \mathrm{U}_{\mathrm{e}}$ of the excited $\left(S t_{D c}=0.85\right)$ and unexcited eiliptic jets. 


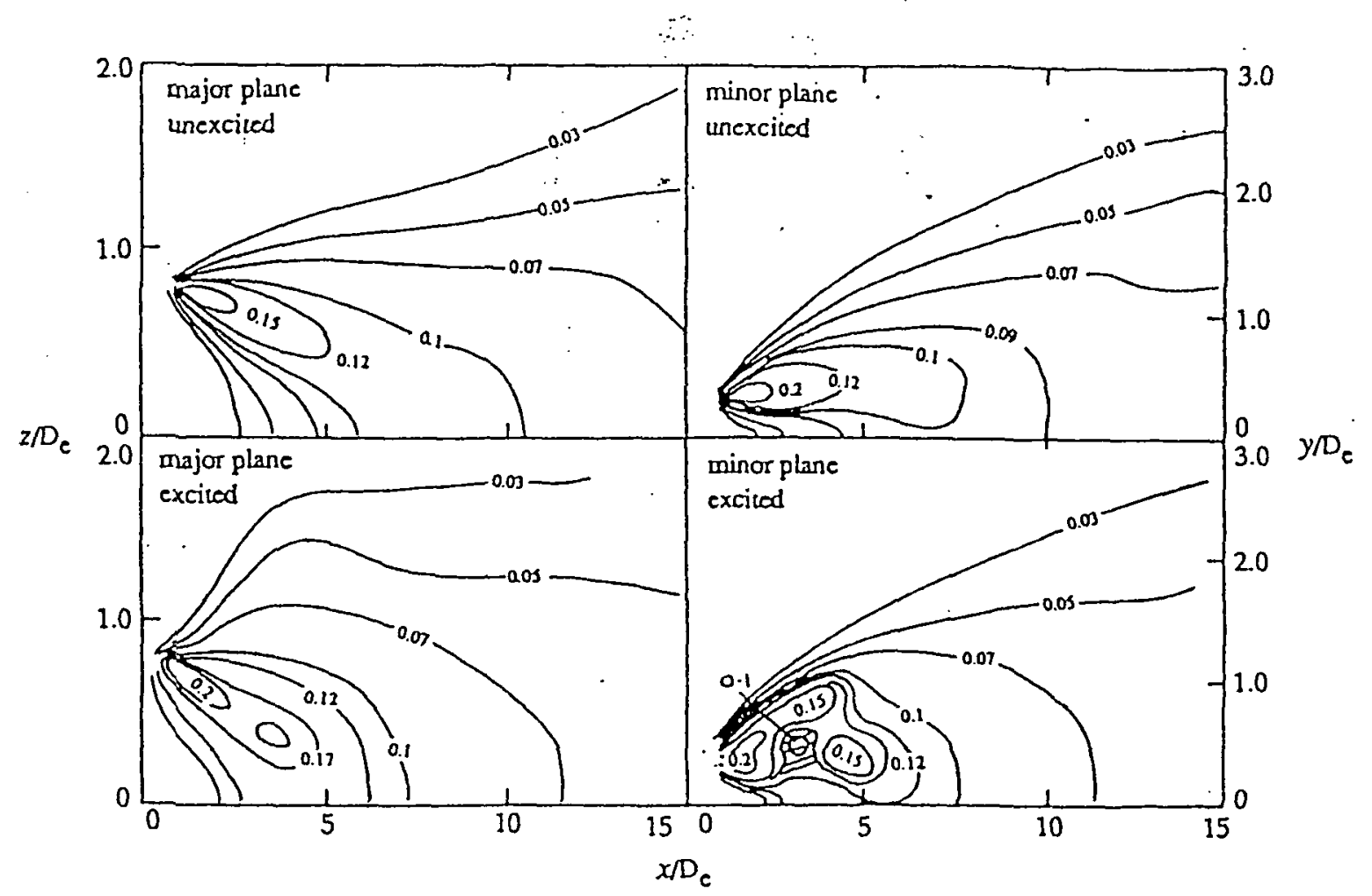

Figure 24 Contours of $v^{\prime} U_{e}$ of the excited $\left(S t_{D e}=0.85\right)$ and unexcited elliptic jets.

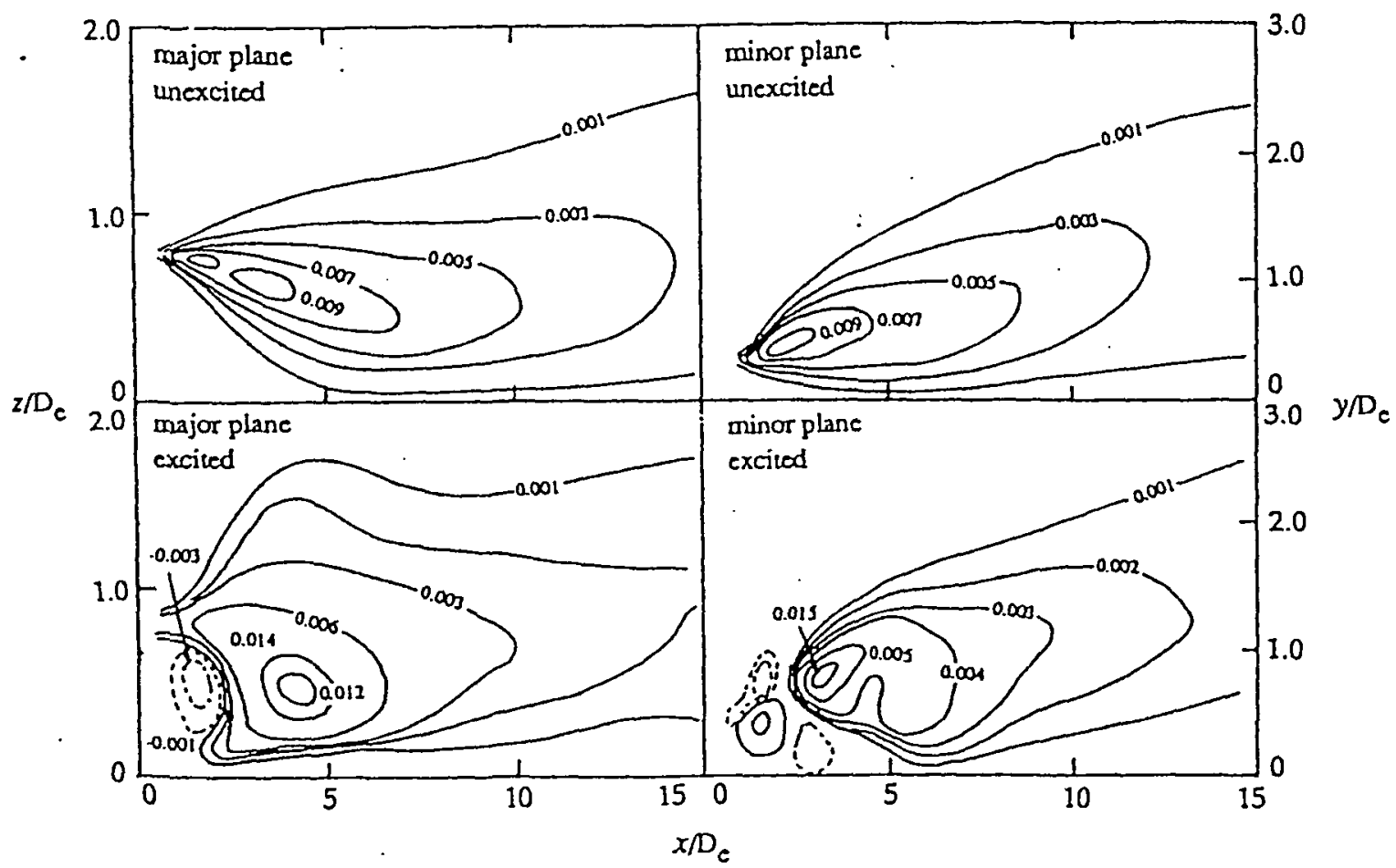

Figure 25 Contours of $u v / \mathrm{U}_{\mathrm{e}}{ }^{2}$ of the excited $\left(S_{\mathrm{De}_{\mathrm{D}}}=0.85\right)$ and unexcited elliptic jets. 
(a)

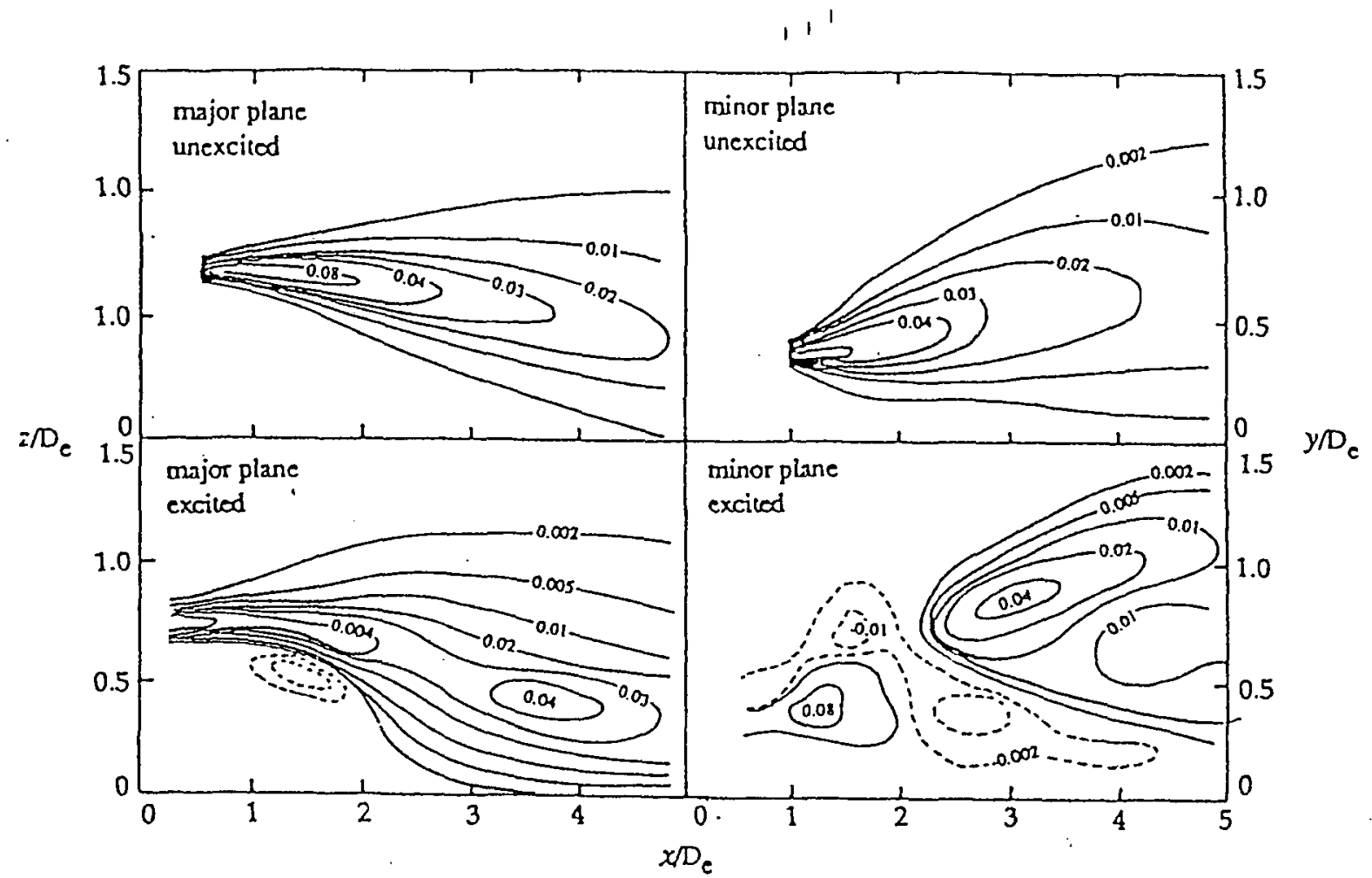

(b)

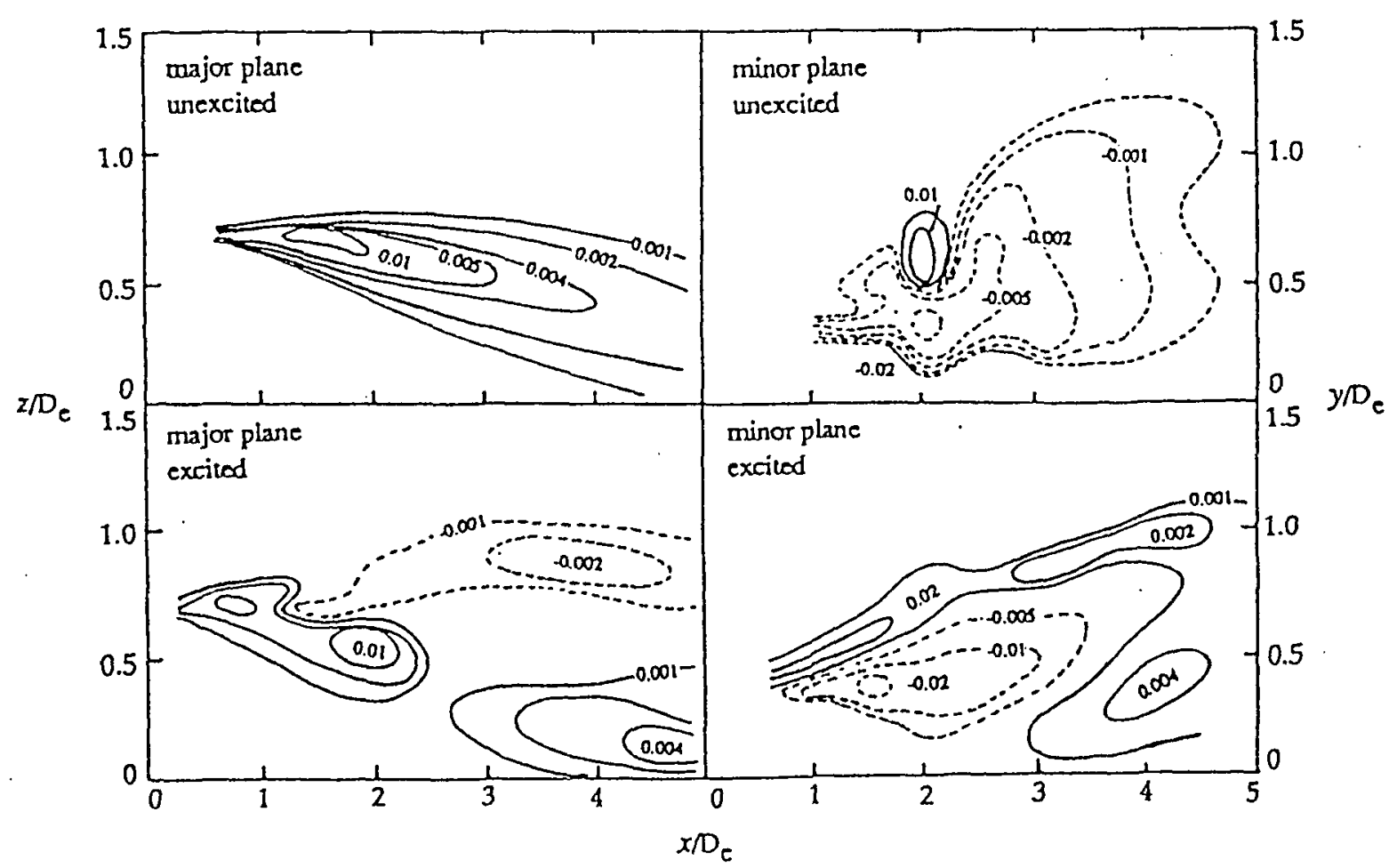

Figure 26 Contours of time-average turbulence production of excited $\left(S t_{D e}=0.85\right.$ ) and unexcited • elliptic jets: (a) shear production; (b) normal production; (c) total production. 


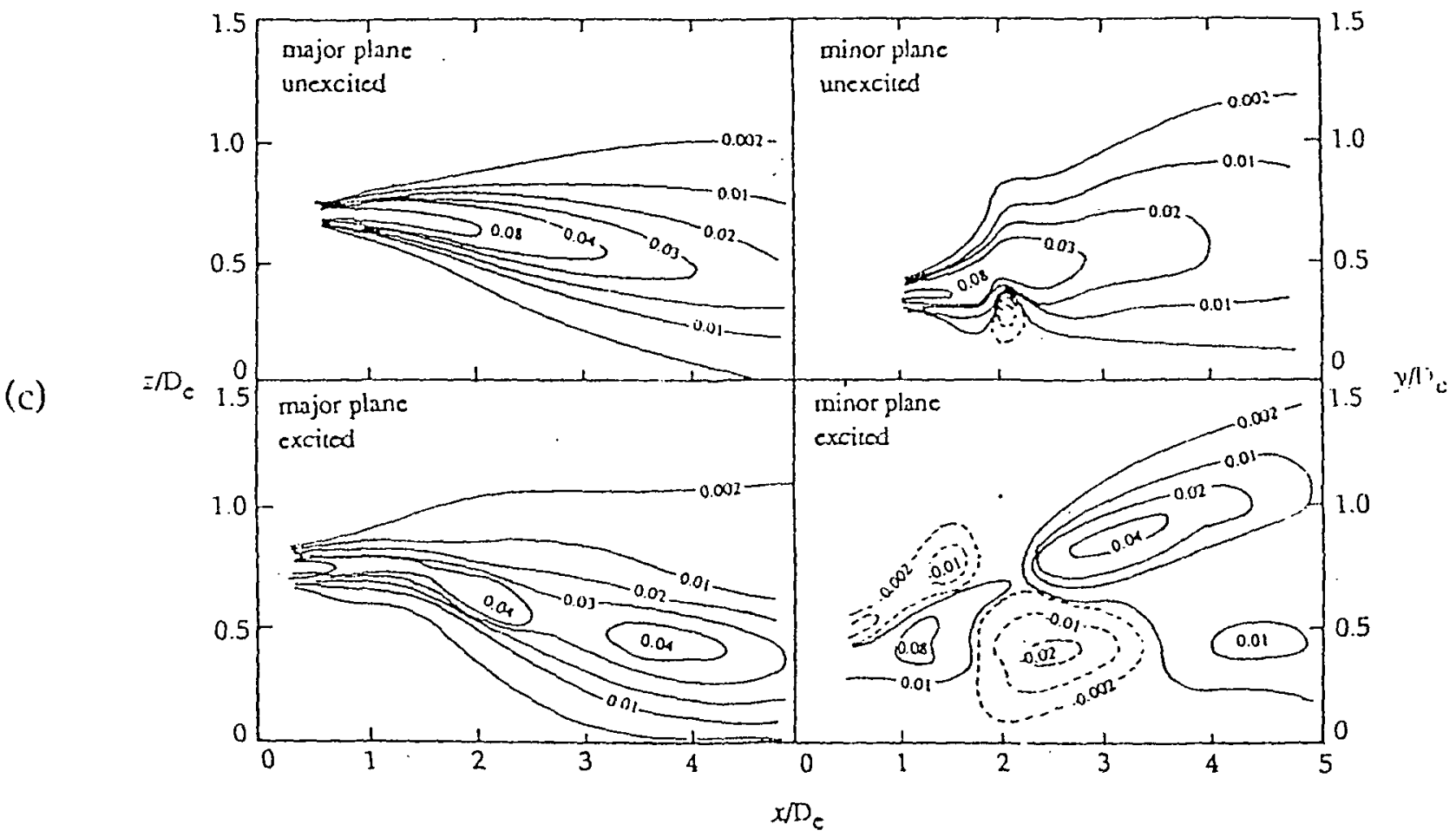

Figure 26(c) 